\title{
KOSZUL DIVISORS ON MODULI SPACES OF CURVES
}

\author{
GAVRIL FARKAS
}

\section{INTRODUCTION}

In this paper we describe a general method of constructing special effective divisors on various moduli spaces using the syzygies of the parametrized objects. The method can be applied to a wide range of moduli problems with the property that the coarse moduli space has canonical singularities hence pluricanonical forms extend over any desingularization of the moduli space. Here we treat the case of the moduli stacks $\overline{\mathcal{M}}_{g, n}$ and we develop the intersection theory machinery necessary to understand the compactification and compute the class of these Koszul divisors.

The idea of using geometric divisors to study the geometry of a moduli space can be traced back to Harris and Mumford (cf. [HM]) who, in the course of their proof that $\overline{\mathcal{M}}_{g}$ is of general type for odd genus $g=2 k-1 \geq 25$, studied the Hurwitz divisor $\mathcal{M}_{g, k}^{1}:=\left\{[C] \in \mathcal{M}_{g}: \exists C \stackrel{k: 1}{\rightarrow} \mathbf{P}^{1}\right\}$ consisting of curves with a pencil $\mathfrak{g}_{k}^{1}$. By computing the class of $\overline{\mathcal{M}}_{g, k}^{1}$ and comparing it in to $K_{\overline{\mathcal{M}}_{g}}$, they showed that when $g \geq 25$, the canonical class is a combination with positive coefficients of $\left[\overline{\mathcal{M}}_{g, k}^{1}\right]$, the Hodge class $\lambda$ (which is big and nef) and various boundary classes. Later, numerous other divisor class calculations were carried out. Eisenbud and Harris considered the Petri divisors on $\overline{\mathcal{M}}_{2 k-2}$ consisting of curves $C$ of genus $2 k-2$ having a pencil $A \in W_{k}^{1}(C)$ which violates the Petri Theorem, which then they used to show that $\overline{\mathcal{M}}_{g}$ is of general type for even $g \geq$ 24 (cf. [EH3]). Logan introduced pointed Brill-Noether divisors on $\overline{\mathcal{M}}_{g, g}$ consisting of marked curves $\left[C, x_{1}, \ldots, x_{g}\right] \in \overline{\mathcal{M}}_{g, g}$ with the property that $h^{0}\left(C, \mathcal{O}_{C}\left(x_{1}+\cdots+x_{g}\right)\right) \geq 2$ (cf. [Log]) and used them to determine the Kodaira type of $\overline{\mathcal{M}}_{g, n}$ for various $g$ and $n$.

More recently, in $[\mathrm{FP}]$ in our work on the Harris-Morrison Slope Conjecture, we reinterpreted the condition that a curve $[C] \in \mathcal{M}_{10}$ lie on a $K 3$ surface into saying that there exists a linear system $L=K_{C}\left(-\mathfrak{g}_{6}^{1}\right) \in W_{12}^{4}(C)$ such that the embedded curve $C \stackrel{|L|}{\hookrightarrow} \mathbf{P}^{4}$ is not projectively normal. Using this description we computed the class of the compactification of the divisor $\mathcal{K}_{10}$ of curves with this property and showed that $s\left(\overline{\mathcal{K}}_{10}\right)=7$, thus contradicting the Slope Conjecture. In [F1] we generalized this construction to cover all cases $g=6 i+10$ and we obtained a (sometimes virtual) Hurwitz type divisor on $\overline{\mathcal{M}}_{6 i+10}$ defined in terms of linear series $\mathfrak{g}_{9 i+12}^{3 i+4}=K_{C}\left(-\mathfrak{g}_{3 p+6}^{1}\right)$ residual to a pencil of minimal degree. This locus, when a divisor, always has slope $<6+12 /(g+1)$ thus violating the Slope Conjecture (see $[\mathrm{HMO}]$ and $[\mathrm{FP}]$ for background on the effective cone of $\overline{\mathcal{M}}_{g}$ and for the significance of the Harris-Morrison Conjecture). Around the

Research partially supported by an Alfred P. Sloan Fellowship, the NSF Grants DMS-0450670 and DMS0500747 and a 2006 Texas Summer Research Assignment. 
same time and using different techniques, Khosla provided a different type of example of a divisor on $\overline{\mathcal{M}}_{g}$ having exceptionally small slope (cf. [Kh]): on $\overline{\mathcal{M}}_{21}$ the closure of the locus of curves $[C] \in \overline{\mathcal{M}}_{21}$ possessing an embedding $C \hookrightarrow \mathbf{P}^{6}$ given by a $\mathfrak{g}_{24}^{6}$ such that $C$ lies on a quadric, is a divisor whose slope is less than the slope of the Harris-Mumford divisor $\overline{\mathcal{M}}_{21,11}^{1}$.

The aims of this paper are (1) to give a unified framework for doing divisor class calculation on $\overline{\mathcal{M}}_{g, n}$ and (2) to provide (empirical) evidence that syzygy divisors may be the answer to the riddle: Given a moduli space, what is the most intrinsic, most natural and from the point of view of birational geometry, most useful effective divisor on it? We prove that virtually all interesting known divisors on $\overline{\mathcal{M}}_{g}$ (the Harris-Mumford divisor, the Petri divisor and all known counterexamples to the Harris-Morrison Conjecture) can be treated in a unified way and are particular instances of a single syzygy type construction. In [F3] we shall further illustrate this ideology by studying moduli spaces of curves with various level structures from the point of view of syzygies.

We fix integers $i \geq 0$ and $s \geq 1$ and set $r:=2 s+s i+i, g:=r s+s$ and $d:=r s+r$. We denote by $\mathfrak{G}_{d}^{r}$ the stack parametrizing pairs $[C, L]$ with $[C] \in \mathcal{M}_{g}$ and $L \in W_{d}^{r}(C)$ and denote by $\sigma: \mathfrak{G}_{d}^{r} \rightarrow \mathcal{M}_{g}$ the natural projection. Since $\rho(g, r, d)=0$, by general BrillNoether theory, the general curve of genus $g$ will have finitely many $\mathfrak{g}_{d}^{r}$ s and there exists a unique irreducible component of $\mathfrak{G}_{d}^{r}$ which maps onto $\mathcal{M}_{g}$. We denote by $K_{i, j}(C, L)$ the $(i, j)$-th Koszul cohomology group of the pair $(C, L)$ and we define a stratification of $\mathfrak{G}_{d}^{r}$ with strata $\mathcal{U}_{g, i}:=\left\{(C, L) \in \mathfrak{G}_{d}^{r}: K_{i, 2}(C, L) \neq 0\right\}$ and set $\mathcal{Z}_{g, i}:=\sigma_{*}\left(\mathcal{U}_{g, i}\right)$.

Theorem 1.1. If $\sigma: \widetilde{\mathfrak{G}}_{d}^{r} \rightarrow \overline{\mathcal{M}}_{g}$ is the compactification of $\mathfrak{G}_{d}^{r}$ given by limit linear series, then there exists a natural morphism between torsion free sheaves of the same rank $\phi: \mathcal{A} \rightarrow \mathcal{B}$ over $\widetilde{\mathfrak{G}}_{d}^{r}$ such that $\overline{\mathcal{Z}}_{g, i}$ is the image of the degeneracy locus of $\phi$. The class of the pushforward to $\overline{\mathcal{M}}_{g}$ of the virtual degeneracy locus of $\phi$ is given by

$$
\sigma_{*}\left(c_{1}(\mathcal{B}-\mathcal{A})\right) \equiv a \lambda-b_{0} \delta_{0}-b_{1} \delta_{1}-\cdots-b_{[g / 2]} \delta_{[g / 2]},
$$

where $a, b_{0}, \ldots, b_{[g / 2]}$ are explicitly given coefficients such that $b_{1}=12 b_{0}-a$ and

$$
s\left(\sigma_{*}\left(c_{1}(\mathcal{B}-\mathcal{A})\right)\right)=\frac{a}{b_{0}}=6 \frac{f(s, i)}{(i+2) s g(s, i)}, \text { with }
$$

$f(s, i)=\left(i^{4}+24 i^{2}+8 i^{3}+32 i+16\right) s^{7}+\left(i^{4}+4 i^{3}-16 i-16\right) s^{6}-\left(i^{4}+7 i^{3}+13 i^{2}-12\right) s^{5}-\left(i^{4}+\right.$ $\left.2 i^{3}+i^{2}+14 i+24\right) s^{4}+\left(2 i^{3}+2 i^{2}-6 i-4\right) s^{3}+\left(i^{3}+17 i^{2}+50 i+41\right) s^{2}+\left(7 i^{2}+18 i+9\right) s+2 i+2$

and

$$
\begin{gathered}
g(s, i)=\left(i^{3}+6 i^{2}+12 i+8\right) s^{6}+\left(i^{3}+2 i^{2}-4 i-8\right) s^{5}-\left(i^{3}+7 i^{2}+11 i+2\right) s^{4}- \\
-\left(i^{3}-5 i\right) s^{3}+\left(4 i^{2}+5 i+1\right) s^{2}+\left(i^{2}+7 i+11\right) s+4 i+2 .
\end{gathered}
$$

Furthermore, we have that $6<\frac{a}{b_{0}}<6+\frac{12}{g+1}$ whenever $s \geq 2$. If the morphism $\phi$ is generically non-degenerate, then $\overline{\mathcal{Z}}_{g, i}$ is a divisor on $\overline{\mathcal{M}}_{g}$ which gives a counterexample to the Slope Conjecture for $g=s(2 s+s i+i+1)$.

Despite its complicated appearance, the slope computed in Theorem 1.1 encodes a surprising amount of information about $\overline{\mathcal{M}}_{g}$, in particular, for suitable choices of $s$ 
and $i$ it specializes to the divisor class calculations carried out in [HM], [EH3], [Kh], $[\mathrm{FP}]$ and [F1] which were originally obtained using a variety of ad hoc techniques. The first interesting case is $s=1, g=2 i+3$ when $\mathfrak{g}_{d}^{r}=\mathfrak{g}_{2 g-2}^{g-1}=K_{C}$ (the canonical bundle is the only $\mathfrak{g}_{2 g-2}^{g-1}$ on a curve of genus $g$ ). We can relate the locus $\mathcal{Z}_{2 i+3, i}$ to more classical loci in $\mathcal{M}_{2 i+3}$ using Green's Conjecture which predicts that for any smooth curve $C$ one has the equivalence $K_{l, 2}\left(C, K_{C}\right)=0 \Leftrightarrow l<\operatorname{Cliff}(C)$. Although Green's Conjecture for arbitrary curves is still open, Voisin proved it for generic curves of given gonality (cf. [V1], [V2]). In our case this gives a set-theoretic identification between $\mathcal{Z}_{2 i+3, i}$ and the locus $\mathcal{M}_{2 i+3, i+2}^{1}$ of $(i+2)$-gonal curves. Thus Theorem 1.1 provides a new way of calculating the class of the compactification of the Brill-Noether divisor first computed by Harris and Mumford (cf. [HM]):

Corollary 1.2. The slope of the Harris-Mumford divisor $\overline{\mathcal{M}}_{2 i+3, i+2}^{1}$ on $\overline{\mathcal{M}}_{2 i+3}$ consisting of curves which cover $\boldsymbol{P}^{1}$ with degree $\leq i+2$ is given by the formula

$$
s\left(\overline{\mathcal{M}}_{2 i+3, i+2}^{1}\right)=\frac{6(i+3)}{i+2}=6+\frac{12}{g+1} .
$$

For $s=2$ and $g=6 i+10$ (that is, in the case $h^{1}(L)=2$ when $\mathfrak{G}_{d}^{r}$ is isomorphic to a Hurwitz stack parametrizing covers of $\mathbf{P}^{1}$ ), we recover the main result from [F1]:

Corollary 1.3. The slope of the divisor $\overline{\mathcal{Z}}_{6 i+10, i}$ on $\overline{\mathcal{M}}_{6 i+10}$ consisting of curves possessing a pencil $\mathfrak{g}_{3 i+6}^{1}$ such that if $L=K_{C}\left(-\mathfrak{g}_{3 i+6}^{1}\right) \in W_{9 i+12}^{3 i+4}(C)$ denotes the residual linear system, then $C \stackrel{|L|}{\hookrightarrow} \boldsymbol{P}^{3 i+4}$ fails to satisfy the Green-Lazarsfeld property $\left(N_{i}\right)$, is given by the formula:

$$
s\left(\overline{\mathcal{Z}}_{6 i+10, i}\right)=\frac{3(4 i+7)\left(6 i^{2}+19 i+12\right)}{\left(12 i^{2}+31 i+18\right)(i+2)} .
$$

In the case $i=0$ we have complete results in the sense that (1) we show that $\overline{\mathcal{Z}}_{g, 0}$ is an actual divisor on $\overline{\mathcal{M}}_{g}$ and (2) we can compute the entire class $\left[\overline{\mathcal{Z}}_{g, 0}\right]$ rather than the $\lambda, \delta_{0}$ and $\delta_{1}$ coefficients. In particular we show that $b_{j} \geq b_{0}$ for $j \geq 1$, hence the slope of $\overline{\mathcal{Z}}_{g, 0}$ is always computed by the $\lambda$ and $\delta_{0}$ coefficients.

Theorem 1.4. For $g=s(2 s+1), r=2 s, d=2 s(s+1)$ the slope of the virtual class of the locus of those curves $[C] \in \overline{\mathcal{M}}_{g}$ for which there exists $L \in W_{d}^{r}(C)$ such that the embedded curve $C \stackrel{|L|}{\hookrightarrow} \boldsymbol{P}^{r}$ sits on a quadric hypersurface, is

$$
s\left(\overline{\mathcal{Z}}_{s(2 s+1), 0}\right)=\frac{a}{b_{0}}=\frac{3\left(16 s^{7}-16 s^{6}+12 s^{5}-24 s^{4}-4 s^{3}+41 s^{2}+9 s+2\right)}{s\left(8 s^{6}-8 s^{5}-2 s^{4}+s^{2}+11 s+2\right)} .
$$

Note that this locus has been first considered by D. Khosla who, using a different approach, was able to compute the coefficients $a$ and $b_{0}$ (cf. [Kh]). Showing that the degeneration loci $\mathcal{Z}_{g, i}$ are actual divisors on $\mathcal{M}_{g}$ can be very difficult in practice (for instance, the statement that $\mathcal{Z}_{2 i+3, i}$ is a divisor on $\mathcal{M}_{2 i+3}$ is essentially Green's Conjecture for a generic curve of odd genus). Apart from the case $s=1$ (settled by Voisin in [V2]), the only cases where it was previously known that $\mathcal{Z}_{g, i}$ is an actual divisor were $s=2, i=0$ (cf. [FP], this being the $K 3$ divisor on $\mathcal{M}_{10}$ ), $s=2, i=1,2$ (cf. [F1]) and when $s=3, i=0$ (cf. [Kh] $)$ - these last three cases having been settled using Macaulay. 
Here we show that for $i=0$ the degeneracy loci $\mathcal{Z}_{g, 0}$ are honest divisors on $\overline{\mathcal{M}}_{g}$, that is, the map $\phi: \mathcal{A} \rightarrow \mathcal{B}$ described in Theorem 1.1 is generically non-degenerate. This provides the first infinite sequence of actual (as opposed to virtual) counterexamples to the conjecture from [HMo]:

Theorem 1.5. For an integer $s \geq 2$ we set $r:=2 s, d:=2 s(s+1)$ and $g:=s(2 s+1)$. Then $\phi: \mathcal{A} \rightarrow \mathcal{B}$ is a generically non-degenerate map between vector bundles over $\widetilde{\mathfrak{G}}_{d}^{r}$ having the same rank and its degeneracy locus

$$
\mathcal{Z}_{g, 0}:=\left\{[C] \in \mathcal{M}_{g}: \exists L \in W_{d}^{r}(C) \text { such that } C \stackrel{|L|}{\hookrightarrow} \boldsymbol{P}^{r} \text { is not projectively normal }\right\}
$$

is a divisor on $\overline{\mathcal{M}}_{g}$ of slope

$$
s\left(\overline{\mathcal{Z}}_{g, 0}\right)=\frac{3\left(16 s^{7}-16 s^{6}+12 s^{5}-24 s^{4}-4 s^{3}+41 s^{2}+9 s+2\right)}{s\left(8 s^{6}-8 s^{5}-2 s^{4}+s^{2}+11 s+2\right)}
$$

contradicting the Slope Conjecture.

As an application of the techniques developed for proving Theorem 1.1 we compute the class of the Gieseker-Petri divisors on $\overline{\mathcal{M}}_{g}$. Recall that Petri's Theorem asserts that for a general curve $[C] \in \mathcal{M}_{g}$ and for an arbitrary line bundle $L$ on $C$, the multiplication map $\mu_{0}(L): H^{0}(L) \otimes H^{0}\left(K_{C} \otimes L^{\vee}\right) \rightarrow H^{0}\left(K_{C}\right)$ is injective (see [EH4] and [Laz] for two very different, relatively short proofs). The map $\mu_{0}(L)$ governs the deformation theory of sections of the line bundle $L$. It is well-known that $W_{d}^{r}(C)$ is smooth of expected dimension $\rho(g, r, d)$ at a point $[L] \in W_{d}^{r}(C)$ if and only if $\mu_{0}(L)$ is injective. The locus in $\mathcal{M}_{g}$ where the Petri Theorem fails breaks up into numerous components and its geometry is still rather mysterious (see [F2], [EH3]). For integers $r, s \geq 1$ we set again $d:=r s+r$ and $g:=r s+s$, so that $\rho(g, r, d)=0$. Like in [F2] we define the Gieseker-Petri locus

$$
\mathcal{G P}_{g, d}^{r}:=\left\{[C] \in \mathcal{M}_{g}: \exists L \in W_{d}^{r}(C) \text { such that } \mu_{0}(L) \text { is not injective }\right\} .
$$

Theorem 1.6. For $d=r s+r$ and $g=r s+s$, the class of the Gieseker-Petri divisor in $\overline{\mathcal{M}}_{g}$ is given by the formula:

$$
\overline{\mathcal{G P}}_{g, d}^{r} \equiv \frac{c_{r}(s-1) r}{(r+s+1)(r s+s-2)(r s+s-1)}\left(a \lambda-b_{0} \delta_{0}-b_{1} \delta_{1}-\sum_{j=2}^{[g / 2]} b_{j} \delta_{j}\right),
$$

where $c_{r}$ is an explicitly given constant defined in Lemma 2.6

$$
\begin{gathered}
a=r^{2} s^{2}(4 s+r+r s+10)+s^{2}(5 r s+24 r+2 s+15)+21 s+26 r s+7 r^{2} s+2 r+2, \\
b_{0}=\frac{s(s+1)(r+1)(r+2)(r s+s+4)}{6}, \\
b_{1}=(r s+s-1)\left(3 r s^{2}+2 s^{2}+r^{2} s^{2}+7 s+6 r s+r^{2} s+2 r+2\right),
\end{gathered}
$$

and $b_{j} \geq b_{1}$ for $j \geq 2$. In particular we have the following expression for the slope:

$$
s\left(\overline{\mathcal{G P}}_{g, d}^{r}\right)=6+\frac{12}{g+1}+\frac{6(s+r+1)(r s+s-2)(r s+s-1)}{s(s+1)(r+1)(r+2)(r s+s+4)(r s+s+1)} .
$$


Theorem 1.6 shows that the Gieseker-Petri divisors satisfy the Slope Conjecture, that is, $s\left(\overline{\mathcal{G P}}_{g, d}^{r}\right) \geq 6+12 /(g+1)$. This is consistent with Proposition 2.2 from [FP] stating that any effective divisor on $\overline{\mathcal{M}}_{g}$ violating the Slope Conjecture would have to contain the locus $\overline{\mathcal{K}}_{g} \subset \overline{\mathcal{M}}_{g}$ of curves lying on $K 3$ surfaces and with Lazarsfeld's result (cf. [Laz]) that a general $[C] \in \overline{\mathcal{K}}_{g}$ satisfies Petri's Theorem. For $s=2$, Theorem 1.6 specializes to Eisenbud and Harris's computation originally used to show that $\overline{\mathcal{M}}_{g}$ is of general type for large even genus (cf. [Ë3], Theorem 2):

Corollary 1.7. For $g=2 r+2$, the Gieseker-Petri divisor $\mathcal{G} \mathcal{P}_{2 r+2,3 r}^{r}$ can be interpreted as the branch locus of the generically finite map $\sigma: \mathfrak{G}_{3 r}^{r} \rightarrow \mathcal{M}_{2 r+2}$ from the Hurwitz stack $\mathfrak{G}_{3 r}^{r}=\mathfrak{G}_{r+2}^{1}$ and one has the following expression for its class:

$$
\overline{\mathcal{G P}}_{2 r+2,3 r}^{r} \equiv c_{r}\left(\frac{6 r^{2}+25 r+20}{2 r+1} \lambda-\frac{(r+1)(r+2)}{2 r+1} \delta_{0}-\delta_{1}-\sum_{j=2}^{r+1} b_{j} \delta_{j}\right),
$$

where $b_{j}>1$ for $j \geq 2$.

In Section 4 we describe five different ways of constructing Koszul divisors on $\overline{\mathcal{M}}_{g, n}$. The direct analogue of Theorem 1.1] in the pointed case is the following statement:

Theorem 1.8. Fix positive integers $g$ and $i$ such that

$$
n:=\frac{2 g+i+1}{2}+\frac{\sqrt{(i+1)^{2}+4 i g+8 g}}{2}
$$

is an integer. Then the locus

$$
\mathfrak{S y}_{\mathfrak{z}}{ }_{g, n}:=\left\{\left[C, x_{1}, \ldots, x_{n}\right] \in \mathcal{M}_{g, n}: K_{i, 2}\left(C, \mathcal{O}_{C}\left(x_{1}+\cdots+x_{n}\right)\right) \neq 0\right\}
$$

is a divisor on $\mathcal{M}_{g, n}$, and the class of its compactification is given by the formula:

$\overline{\mathfrak{S} \mathfrak{y}}_{g, n} \equiv \frac{1}{n-g-i}\left(\begin{array}{c}n-g-1 \\ i\end{array}\right)\left(-(n+g-1) \lambda+(3 g-n+i+1) \sum_{j=1}^{n} \psi_{j}-0 \cdot \delta_{i r r}-\sum_{j, t \geq 0} \sum_{|S|=t} b_{j: t} \delta_{j: S}\right)$,

where $b_{j: t}>1$ are explicitly determined coefficients.

Another infinite sequence of interesting divisors on $\overline{\mathcal{M}}_{g, n}$ can be obtained by using the Gaussian-Wahl map associated to a line bundle on a curve. Recall that if $L$ is a line bundle on a curve $C$, the Wahl map $\psi_{L}: \wedge^{2} H^{0}(L) \rightarrow H^{0}\left(K_{C} \otimes L^{\otimes 2}\right)$ is defined by the formula $\psi_{L}(f \wedge g):=f \cdot d g-g \cdot d f$. It is known that the divisor $\overline{\mathcal{Z}}_{10,0}$ on $\overline{\mathcal{M}}_{10}$ can be viewed as the global degeneracy locus corresponding to the Wahl map of the canonical curve (see [FP] for details and further references). If $\left[C, x_{1}, \ldots, x_{n}\right] \in \mathcal{M}_{g, n}$, we set $\Gamma:=x_{1}+\cdots+x_{n} \in C_{n}$.

Theorem 1.9. Fix an integer $g$ such that

$$
n:=\frac{2 g+3+\sqrt{24 g+1}}{2}
$$

is an integer. Then the locus

$\mathfrak{W a h h l}_{g, n}:=\left\{\left[C, x_{1}, \ldots, x_{n}\right] \in \mathcal{M}_{g, n}: \psi_{\Gamma}: \wedge^{2} H^{0}\left(\mathcal{O}_{C}(\Gamma)\right) \rightarrow H^{0}\left(K_{C} \otimes \mathcal{O}_{C}(2 \Gamma)\right)\right.$ is degenerate $\}$ 
is a divisor on $\mathcal{M}_{g, n}$ and its compactification has the following class:

$$
\overline{\mathfrak{W} a \mathfrak{h l}}_{g, n}=-(n-g-1) \lambda+(n-g-1) \sum_{j=1}^{n} \psi_{j}-\delta_{i r r}-\sum_{j, t \geq 0} b_{j: t} \sum_{|S|=t} \delta_{j: S},
$$

where $b_{j: t}>1$ are explicitly determined coefficients.

Note that although the divisors $\overline{\mathfrak{S y g}}_{g, n}$ and $\overline{\mathfrak{W a h l}}_{g, n}$ live on $\overline{\mathcal{M}}_{g, n}$ 's for some very particular choices of $n$, using the forgetful and clutching maps $\overline{\mathcal{M}}_{g, n} \rightarrow \overline{\mathcal{M}}_{g, n-1}$ and $\overline{\mathcal{M}}_{i, n_{1}} \times \overline{\mathcal{M}}_{g-i, n_{2}} \rightarrow \overline{\mathcal{M}}_{g, n_{1}+n_{2}}$ one immediately has explicit Koszul divisors on $\overline{\mathcal{M}}_{g, n}$ for all $g$ and $n$. Among the other syzygetic ways of producing divisors on $\overline{\mathcal{M}}_{g, n}$ we single the one using the Minimal Resolution Conjecture (cf. Theorem 4.2), which can be thought of as a generalization of the divisor of higher Weierstrass points and which is especially useful in the case of a large number of marked points. An immediate application of the calculations in Section 4 is the following result about the Kodaira type of $\overline{\mathcal{M}}_{g, n}$ :

Theorem 1.10. For integers $g=4, \ldots, 21$, the moduli space $\overline{\mathcal{M}}_{g, n}$ is of general type for all $n \geq f(g)$ where $f(g)$ is described in the following table.

\begin{tabular}{c|cccccccccccccccccc}
$g$ & 4 & 5 & 6 & 7 & 8 & 9 & 10 & 11 & 12 & 13 & 14 & 15 & 16 & 17 & 18 & 19 & 20 & 21 \\
\hline$f(g)$ & 16 & 15 & 16 & 15 & 14 & 13 & 11 & 12 & 13 & 11 & 10 & 10 & 9 & 9 & 9 & 7 & 6 & 4
\end{tabular}

This result represents an improvement of Logan's Theorem 5.1 for $g=4-6,10$, $14-16,18-22$, the entries for the remaining values of $g$ being those from [Log].

Acknowledgment: I am grateful to Sean Keel for many discussions over the years on topics related to this circle of ideas.

\section{CONSTRUCTING DIVISORS OF SMALL SLOPE USING SYZYGIES}

For a projective variety $X$ and a line bundle $L$ on $X$ we denote by $K_{i, j}(X, L)$ the Koszul cohomology group obtained from the complex

$$
\wedge^{i+1} H^{0}(L) \otimes H^{0}\left(L^{\otimes(j-1)}\right) \longrightarrow \wedge^{i} H^{0}(L) \otimes H^{0}\left(L^{\otimes j}\right) \longrightarrow \wedge^{i-1} H^{0}(L) \otimes H^{0}\left(L^{\otimes(j+1)}\right),
$$

where the maps are the Koszul differentials (cf. [Gr]). Assume $L$ is globally generated and $M_{L}$ is the vector bundle on $X$ defined by the exact sequence

$$
0 \rightarrow M_{L} \rightarrow H^{0}(L) \otimes \mathcal{O}_{X} \rightarrow L \rightarrow 0 .
$$

A simple argument using the exact sequences

$$
0 \longrightarrow \wedge^{a} M_{L} \otimes L^{\otimes b} \rightarrow \wedge^{a} H^{0}(L) \otimes L^{\otimes b} \longrightarrow \wedge^{a-1} M_{L} \otimes L^{\otimes(b+1)} \longrightarrow 0
$$

for various $a$ and $b$, shows that there is an identification

$$
K_{a, b}(X, L)=\frac{H^{0}\left(\wedge^{a} M_{L} \otimes L^{\otimes b}\right)}{\text { Image }\left\{\wedge^{a+1} H^{0}(L) \otimes H^{0}\left(L^{\otimes(b-1)}\right)\right\}} .
$$


¿From now on we fix integers $i \geq 0$ and $s \geq 1$ and set

$$
r:=2 s+s i+i, g:=r s+s, \text { and } d:=r s+r .
$$

We introduce the open substack $\mathcal{M}_{g}^{0}$ of $\mathcal{M}_{g}$ corresponding to curves $[C] \in \mathcal{M}_{g}$ such that $W_{d-1}^{r}(C)=\emptyset$ and $W_{d}^{r+1}(C)=\emptyset$. Then $\operatorname{codim}\left(\mathcal{M}_{g}-\mathcal{M}_{g}^{0}, \mathcal{M}_{g}\right) \geq 3$. We denote by $\mathfrak{P i c}^{d}$ the degree $d$ Picard stack over $\mathcal{M}_{g}$ (precisely, the étale sheafification of the Picard functor). In particular if $\mathrm{Pic}_{\mathrm{M}_{\mathrm{g}}}^{\mathrm{d}}$ is the coarse moduli space associated to $\mathfrak{P i c}^{d}$, then for any $\mathcal{M}_{g}$-scheme $T \rightarrow \mathcal{M}_{g}$ originating from a family of genus $g$ curves $\mathcal{X} \rightarrow T$, the fibre product $T \times{ }_{M_{g}} \operatorname{Pic}_{M_{\mathrm{g}}}^{\mathrm{d}}$ is the relative Picard algebraic space $\mathrm{Pic}_{\mathcal{X} / \mathrm{T}}^{\mathrm{d}}$. We denote by $\mathfrak{G}_{d}^{r} \subset \mathfrak{P i c}^{d}$ the stack parametrizing pairs $[C, L]$ with $[C] \in \mathcal{M}_{g}$ and $L \in W_{d}^{r}(C)$ and by $\sigma: \mathfrak{G}_{d}^{r} \rightarrow \mathcal{M}_{g}$ the natural projection. Since $\rho(g, r, d)=0$, by general Brill-Noether theory, the general curve of genus $g$ will have finitely many $\mathfrak{g}_{d}^{r}$ s $\mathrm{s}$ and there exists a unique irreducible component of $\mathfrak{G}_{d}^{r}$ which maps onto $\mathcal{M}_{g}$. Moreover, the image of any component of $\mathfrak{G}_{d}^{r}$ having dimension $\geq 3 g-2$ is a substack of codimension $\geq 2$ in $\mathcal{M}_{g}$ (cf. Corollary 2.5), thus one can ignore these extraneous components of $\mathfrak{G}_{d}^{r}$ when doing divisor class calculations on $\overline{\mathcal{M}}_{g}$.

We shall define a determinantal substack of $\mathfrak{G}_{d}^{r}$ consisting of those pairs $(C, L)$ satisfying the condition $K_{i, 2}(C, L) \neq 0$. We denote by $\pi: \mathcal{M}_{g, 1} \rightarrow \mathcal{M}_{g}$ the universal curve and by $\mathcal{L}$ a universal Poincaré bundle on the fibre product $\mathcal{M}_{g, 1} \times \mathcal{M}_{g} \mathfrak{G}_{d}^{r}$ (In the case such an $\mathcal{L}$ does not exist, we pass to an étale surjection $\Sigma \rightarrow \mathfrak{G}_{d}^{r}$ such that $\Sigma$ is a scheme and $\mathcal{M}_{g, 1} \times \Sigma$ admits a Poincaré bundle and we carry out the construction at this level. In the end our construction will not depend on the choice of $\Sigma$, see also [Est2]). If $p_{1}: \mathcal{M}_{g, 1} \times \mathcal{M}_{g} \mathfrak{G}_{d}^{r} \rightarrow \mathcal{M}_{g, 1}$ and $p_{2}: \mathcal{M}_{g, 1} \times \mathcal{M}_{g} \mathfrak{G}_{d}^{r} \rightarrow \mathfrak{G}_{d}^{r}$ are the natural projections, then $\mathcal{E}:=p_{2 *}\left(\mathcal{L}^{\vee}\right)$ is a vector bundle of rank $r+1$ and there is a tautological embedding of the pullback of the universal curve $\mathcal{M}_{g, 1} \times \mathcal{M}_{g} \mathfrak{G}_{d}^{r}$ into the projective bundle $u: \mathbf{P}(\mathcal{E}) \rightarrow \mathfrak{G}_{d}^{r}$. We define the vector bundle $\mathcal{F}$ on $\mathbf{P}(\mathcal{E})$ by the sequence

$$
0 \longrightarrow \mathcal{F} \rightarrow u^{*}(\mathcal{E}) \longrightarrow \mathcal{O}_{\mathbf{P}(\mathcal{E})}(1) \longrightarrow 0
$$

and we further introduce two vector bundles $\mathcal{A}$ and $\mathcal{B}$ over $\mathfrak{G}_{d}^{r}$ by setting

$$
\mathcal{A}:=u_{*}\left(\wedge^{i} \mathcal{F} \otimes \mathcal{O}_{\mathbf{P}(\mathcal{E})}(2)\right) \text {, and } \mathcal{B}:=u_{*}\left(\wedge^{i} \mathcal{F} \otimes \mathcal{O}_{\mathcal{M}_{g, 1} \times \mathcal{M}_{g} \mathfrak{G}_{d}^{r}}(2)\right) \text {. }
$$

If $C \stackrel{|L|}{\hookrightarrow} \mathbf{P}^{r}$ is the embedding corresponding to a point $(C, L) \in \mathfrak{G}_{d^{\prime}}^{r}$, then

$$
\mathcal{A}(C, L)=H^{0}\left(\mathbf{P}^{r}, \wedge^{i} M_{\mathbf{P}^{r}}(2)\right) \text { and } \mathcal{B}(C, L)=H^{0}\left(C, \wedge^{i} M_{L} \otimes L^{2}\right)
$$

and there is a vector bundle morphism $\phi: \mathcal{A} \rightarrow \mathcal{B}$ given by restriction. Grauert's Theorem guarantees that both $\mathcal{A}$ and $\mathcal{B}$ are vector bundles over $\mathfrak{G}_{d}^{r}$ and their ranks are

$$
\operatorname{rank}(\mathcal{A})=(i+1)\left(\begin{array}{c}
r+2 \\
i+2
\end{array}\right) \text { and } \operatorname{rank}(\mathcal{B})=\left(\begin{array}{c}
r \\
i
\end{array}\right)\left(-\frac{i d}{r}+2 d+1-g\right)
$$

(use that $M_{L}$ is a stable bundle, see [F1], Prop. 2.1, which implies that $H^{1}\left(\wedge^{i} M_{L} \otimes L^{\otimes 2}\right)=$ 0 , hence $\operatorname{rank}(\mathcal{B})$ can be computed from Riemann-Roch). Because of the way we choose $g, r$ and $d$ we can easily check that $\operatorname{rank}(\mathcal{A})=\operatorname{rank}(\mathcal{B})$.

Theorem 2.1. The cycle $\mathcal{U}_{g, i}:=\left\{(C, L) \in \mathfrak{G}_{d}^{r}: K_{i, 2}(C, L) \neq 0\right\}$ is the degeneracy locus of vector bundle map $\phi: \mathcal{A} \rightarrow \mathcal{B}$ over $\mathfrak{G}_{d}^{r}$.

Proof. Along the same lines as the proof of Proposition 2.5 in [F1]. 
Thus $\mathcal{Z}_{g, i}:=\sigma_{*}\left(\mathcal{U}_{g, i}\right)$ is a virtual divisor on $\mathcal{M}_{g}$ when $g=s(2 s+s i+i+1)$.

Remark 2.2. Using (1) it is easy to prove that for every $(C, L) \in \mathfrak{G}_{d}^{r}$ one have the vanishing of Koszul cohomology groups $K_{a, 0}(C, L)=0$ for all $a \geq 1$ and $K_{a, b}(C, L)=0$ for all $b \geq 3$. Thus the only non-trivial Koszul type conditions one could impose on $\mathfrak{G}_{d}^{r}$ involve the groups $K_{a, 1}(C, L)$ and $K_{a, 2}(C, L)$. Because $M_{L}$ is a stable vector bundle on $C$, it is straightforward to show using (1) that

$$
\operatorname{dim} K_{i, 2}(C, L)-\operatorname{dim} K_{i+1,1}(C, L)=\left(\begin{array}{c}
r \\
i
\end{array}\right)\left(2 d-\frac{i d}{r}+1-g\right)-(i+1)\left(\begin{array}{c}
r+2 \\
i+2
\end{array}\right) .
$$

For our choices of $g, r$ and $d$, it follows that $\operatorname{dim} K_{i+1,1}(C, L)=\operatorname{dim} K_{i, 2}(C, L)$, hence $\mathcal{U}_{g, i}$ can also be defined as the locus where $K_{i+1,1}(C, L)$ fails to vanish. This shows that, at least in the case of curves, there are no other Koszul divisors except $\mathcal{U}_{g, i}$.

To prove Theorem 1.1 we shall extend the determinantal structure of $\mathcal{Z}_{g, i}$ over a substack of $\overline{\mathcal{M}}_{g}$ whose complement has codimension $\geq 2$. We denote by $\overline{\mathcal{M}}_{g}:=$ $\mathcal{M}_{g}^{0} \cup\left(\cup_{j=0}^{[g / 2]} \Delta_{j}^{0}\right)$ the locally closed substack of $\overline{\mathcal{M}}_{g}$ obtained by adding to $\mathcal{M}_{g}^{0}$ the open subsets $\Delta_{j}^{0} \subset \Delta_{j}$ for $1 \leq j \leq[\mathrm{g} / 2]$ consisting of 1-nodal genus $g$ curves $C \cup_{y} D$, with $[C] \in \mathcal{M}_{g-j}$ and $[D, y] \in \mathcal{M}_{j, 1}$ being Brill-Noether general curves, and the locus $\Delta_{0}^{0} \subset$ $\Delta_{0}$ containing 1-nodal irreducible genus $g$ curves $C^{\prime}=C / q \sim y$, where $[C, q] \in \mathcal{M}_{g-1}$ is a Brill-Noether general pointed curve and $y \in C$, together with their degenerations consisting of unions of a smooth genus $g-1$ curve and a nodal rational curve. One can then extend the finite covering $\sigma: \mathfrak{G}_{d}^{r} \rightarrow \mathcal{M}_{g}^{0}$ to a proper, generically finite map

$$
\sigma: \widetilde{\mathfrak{G}}_{d}^{r} \rightarrow \widetilde{\mathcal{M}}_{g}
$$

by letting $\widetilde{\mathfrak{G}}_{d}^{r}$ be the space of limit $\mathfrak{g}_{d}^{r}$ s on the curves from $\widetilde{\mathcal{M}}_{g}$ whose dual graph is a tree (see [EH1], Theorem 3.4 for the construction of the variety of limit linear series and also [Oss] for a more functorial approach which eventually leads to the Eisenbud-Harris space). Strictly speaking, Eisenbud and Harris have only constructed the space of refined limit $\mathfrak{g}_{d}^{r}$ s. Using the observation that when $\rho(g, r, d)=0$ every crude non-refined limit $\mathfrak{g}_{d}^{r}$ on a curve of compact type $C \cup_{y} D$, where $[C] \in \mathcal{M}_{j}$ and $[D] \in \mathcal{M}_{g-j}$ can be canonically interpreted as a refined limit $\mathfrak{g}_{d}^{r}$ on the pre-stable curve $C \cup_{y_{1}} \mathbf{P}^{1} \cup_{y_{2}} D$ obtained from $C \cup_{y} D$ by inserting a single $\mathbf{P}^{1}$ at the node $y$, their construction can be easily adapted to cover the case of crude $\mathfrak{g}_{d}^{r \prime}$ s as well. Note that since all limit $\mathfrak{g}_{d}^{r \prime}$ s are dimensionally proper (cf. [EH1], Corollary 3.7), every limit linear series from $\widetilde{\mathfrak{G}}_{d}^{r}$ is smoothable.

To compute the class $\left[\overline{\mathcal{Z}}_{g, i}\right]$, we intersect $\overline{\mathcal{Z}}_{g, i}$ with test curves in the boundary of $\overline{\mathcal{M}}_{g}$ which are defined as follows: we fix a Brill-Noether general curve $C$ of genus $g-1$, a general point $q \in C$ and a general elliptic curve $E$. We define two 1-parameter families

$$
C^{0}:=\{C / y \sim q: y \in C\} \subset \Delta_{0} \subset \overline{\mathcal{M}}_{g} \text { and } C^{1}:=\left\{C \cup_{y} E: y \in C\right\} \subset \Delta_{1} \subset \overline{\mathcal{M}}_{g} .
$$

It is well-known that these families intersect the generators of $\operatorname{Pic}\left(\overline{\mathcal{M}}_{g}\right)$ as follows:

$$
\begin{gathered}
C^{0} \cdot \lambda=0, C^{0} \cdot \delta_{0}=-(2 g-2), C^{0} \cdot \delta_{1}=1 \text { and } C^{0} \cdot \delta_{a}=0 \text { for } a \geq 2, \text { and } \\
C^{1} \cdot \lambda=0, C^{1} \cdot \delta_{0}=0, C^{1} \cdot \delta_{1}=-(2 g-4), C^{1} \cdot \delta_{a}=0 \text { for } a \geq 2 .
\end{gathered}
$$


Next, we fix $2 \leq j \leq[g / 2]$, a general curve $C$ of genus $j$ and a general curve pointed curve $(D, y)$ of genus $g-j$. We define the 1-parameter family $C^{j}:=\left\{C \cup_{y} D: y \in C\right\} \subset$ $\Delta_{j} \subset \overline{\mathcal{M}}_{g}$. We have that

$$
C^{j} \cdot \lambda=0, C^{j} \cdot \delta_{a}=0 \text { for } a \neq j \text { and } C^{j} \cdot \delta_{j}=-(2 j-2) .
$$

We review the notation used in the theory of limit linear series (see [EH1]). If $X$ is a tree-like curve and $l$ is a limit $\mathfrak{g}_{d}^{r}$ on $X$, for a component $Y$ of $X$ we denote by $l_{Y}=$ $\left(L_{Y}, V_{Y} \subset H^{0}\left(L_{Y}\right)\right)$ the $Y$-aspect of $l$. For a point $y \in Y$ we denote by $\left\{a_{i}^{l_{Y}}(y)\right\}_{i=0 \ldots r}$ the vanishing sequence of $l$ at $y$, by $\left\{\alpha_{i}^{l_{Y}}(y)=a_{i}^{l_{Y}}(y)-i\right\}_{i=0 \ldots r}$ the ramification sequence and by $\rho\left(l_{Y}, y\right):=\rho(g, r, d)-\sum_{i=0}^{r} \alpha_{i}^{l_{Y}}(y)$ the adjusted Brill-Noether number with respect to $y$.

Proposition 2.3. (1) Let $C_{y}^{1}=C \cup_{y} E$ be an element of $\Delta_{1}^{0}$. If $\left(l_{C}, l_{E}\right)$ is a limit $\mathfrak{g}_{d}^{r}$ on $C_{y}^{1}$, then $V_{C}=H^{0}\left(L_{C}\right)$ and $L_{C} \in W_{d}^{r}(C)$ has a cusp at $y$. If $y \in C$ is a general point, then $l_{E}=\left(\mathcal{O}_{E}(d y),(d-r-1) y+|(r+1) y|\right)$, that is, $l_{E}$ is uniquely determined. If $y \in C$ is one of the finitely many points for which there exists $L_{C} \in W_{d}^{r}(C)$ such that $\rho\left(L_{C}, y\right)=-1$, then $l_{E}(-(d-r-2) y)$ is a $\mathfrak{g}_{r+2}^{r}$ with vanishing sequence at $y$ being $\geq(0,2,3, \ldots, r, r+2)$. Moreover, at the level of 1-cycles we have the identification $\sigma^{*}\left(C^{1}\right) \equiv X+\nu T$, where

$$
X:=\left\{(y, L) \in C \times W_{d}^{r}(C): h^{0}(C, L(-2 y)) \geq r\right\},
$$

$T \cong \boldsymbol{P}\left(H^{0}\left(\mathcal{O}_{E}((r+2) y)\right) / H^{0}\left(\mathcal{O}_{E}(r y)\right)\right)$ is the curve consisting of $\mathfrak{g}_{r+2}^{r}$ 's on $E$ with vanishing $\geq(0,2, \ldots, r, r+2)$ at the fixed point $y \in E$ and $\nu$ is an explicitly known positive integer.

(2) Let $C_{y}^{0}=C / y \sim q$ be an element of $\Delta_{0}^{0}$. Then limit linear series of type $\mathfrak{g}_{d}^{r}$ on $C_{y}^{0}$ are in 1:1 correspondence with complete linear series $L$ on $C$ of type $\mathfrak{g}_{d}^{r}$ satisfying the condition $h^{0}\left(C, L \otimes \mathcal{O}_{C}(-y-q)\right)=h^{0}(C, L)-1$. There is an isomorphism between the cycle $\sigma^{*}\left(C^{0}\right)$ of $\mathfrak{g}_{d}^{r \prime}$ s on all curves $C_{y}^{0}$ with $y \in C$ and the smooth curve

$$
Y:=\left\{(y, L) \in C \times W_{d}^{r}(C): h^{0}(C, L(-y-q)) \geq r\right\} .
$$

Proof. Part (1) is similar to the proof of Proposition 3.3 from [F1] and we omit the details. For part (2), we claim that for any limit $\mathfrak{g}_{d}^{r}$ on a curve $C_{y}^{0}$ where $y \in C$, the underlying torsion free sheaf is actually locally free. Indeed, otherwise the underlying sheaf would be of the form $\nu_{*}(L)$, where $\nu: C \rightarrow C_{0}^{y}$ is the normalization map and $L \in W_{d-1}^{r}(C)$. But $[C] \in \mathcal{M}_{g-1}$ is assumed to be Brill-Noether general, hence $W_{d-1}^{r}(C)=\emptyset$.

Throughout this paper we routinely use basic facts from Schubert calculus which we briefly recall. If $\mathbf{G}(r, d)$ denotes the Grassmannian of $r$-planes in $\mathbf{P}^{d}$ and

$$
\mathbb{C}^{d+1}=V_{0} \supset V_{1} \supset \cdots \supset V_{r+1}=0
$$

is a decreasing flag, then for any Schubert index $0 \leq \alpha_{0} \leq \ldots \leq \alpha_{r} \leq d-r$, we define the Schubert cycle

$$
\sigma_{\left(\alpha_{0}, \ldots, \alpha_{r}\right)}:=\left\{\Lambda \in \mathbf{G}(r, d): \operatorname{dim}\left(\Lambda \cap V_{\alpha_{i}+i}\right) \geq r+1-i, \text { for } i=0, \ldots, r\right\} .
$$

(This differs slightly from the standard notation from e.g. [FuPr], but it seems better suited for dealing with ramification sequences of linear series). Often we use the fact 
that if $\left(\alpha_{0}, \ldots, \alpha_{r}\right)$ is a Schubert index and $g$ is an integer such that $r g+\sum_{i=0}^{r} \alpha_{i}=$ $(r+1)(d-r)$, then there is an identity in $H^{*}(\mathbf{G}(r, d))$ :

$$
\sigma_{\left(\alpha_{0}, \ldots, \alpha_{r}\right)} \cdot \sigma_{(0,1, \ldots, 1)}^{g}=g ! \frac{\prod_{i<j}\left(\alpha_{j}-\alpha_{i}+j-i\right)}{\prod_{i=0}^{r}\left(g-d+i+\alpha_{i}+r\right) !} .
$$

Proposition 2.4. Let $[C] \in \mathcal{M}_{j}$ be a general curve with $g-2 \geq j \geq[g / 2]$ and $C^{j} \subset \Delta_{j} \subset \overline{\mathcal{M}}_{g}$ the associated test curve of type $(j, g-j)$. Then one has the following equality of 1-cycles in $\widetilde{\mathfrak{G}}_{d}^{r}$ :

$$
\sigma^{*}\left(C^{j}\right)=\sum_{\left(\alpha_{0}, \ldots, \alpha_{r}\right) \in \mathcal{P}_{1}} N_{g-j, \alpha} \cdot X_{j, \alpha}+\sum_{\left(\beta_{0}, \ldots, \beta_{r}\right) \in \mathcal{P}_{2}} M_{j, \beta} \cdot Y_{g-j, \beta}+\sum_{\left(\beta_{0}, \ldots, \beta_{r}\right) \in \mathcal{P}_{3}} Q_{g-j, \beta} \cdot U_{j, \beta},
$$

where we introduce the following notations: $\mathcal{P}_{1}:=\left\{\left(0 \leq \alpha_{0} \leq \ldots \leq \alpha_{r} \leq s\right): \sum_{i=0}^{r} \alpha_{i}=j\right\}$,

$$
\begin{gathered}
\mathcal{P}_{2}:=\left\{\left(0 \leq \beta_{0} \leq \ldots \leq \beta_{r} \leq s+1\right): \sum_{i=0}^{r} \beta_{i}=j+1, \beta_{r-1} \leq s\right\}, \\
\mathcal{P}_{3}:=\left\{\left(0=\beta_{0}<\beta_{1} \leq \ldots \leq \beta_{r} \leq s+1\right): \sum_{i=0}^{r} \beta_{i}=r+1+j\right\}, \\
M_{j, \beta}:=\sigma_{(0,1, \ldots, 1)}^{j} \cdot \sigma_{\left(\beta_{0}, \ldots, \beta_{r}\right)} \in H^{*}(\boldsymbol{G}(r, r+j)) \text { for } \beta \in \mathcal{P}_{2}, \\
N_{g-j, \alpha}:=\sigma_{(0,1, \ldots, 1)}^{g-j} \cdot \sigma_{\left(j-\alpha_{r}, \ldots, j-\alpha_{0}\right)} \in H^{*}(\boldsymbol{G}(r, d)) \text { for } \alpha \in \mathcal{P}_{1}, \\
Q_{g-j, \beta}:=\sigma_{(0,1, \ldots, 1)}^{g-j} \cdot \sigma_{\left(j+1-\beta_{r}, \ldots, j+1-\beta_{1}, j+1\right)} \in H^{*}(\boldsymbol{G}(r, d)) \text { for } \beta \in \mathcal{P}_{3}, \\
X_{j, \alpha}:=\left\{\left(y, L_{C}\right) \in C \times \operatorname{Pic}^{r+j}(C): \alpha_{i}^{L_{C}}(y) \geq \alpha_{i} \text { for } i=0 \ldots r\right\}, \alpha \in \mathcal{P}_{1}, \\
Y_{g-j, \beta}:=\left\{l_{D} \in G_{d}^{r}(D): \alpha_{i}^{l_{D}}(y) \geq j-\beta_{r-i} \text { for } i=0 \ldots r\right\}, \beta \in \mathcal{P}_{2}, \\
U_{j, \beta}:=\left\{\left(y, l_{C}\right) \in C \times G_{r+j+1}^{r}(C): \alpha^{l_{C}}(y) \geq\left(0, \beta_{1}, \ldots, \beta_{r}\right)\right\}, \text { for } \beta \in \mathcal{P}_{3} .
\end{gathered}
$$

Proof. Suppose that $l=\left(l_{C}, l_{D}\right)$ is a limit $\mathfrak{g}_{d}^{r}$ on $C \cup_{y} D$. It is easy to see that the generic point of any component of $\sigma^{*}\left(C^{j}\right)$ corresponds to a refined limit $\mathfrak{g}_{d^{\prime}}^{r}$, so we may assume that $l$ is refined as well. If $\left(\alpha_{0}, \ldots, \alpha_{r}\right)$ is the ramification sequence of $l_{C}$ at $y$, then the condition that $[D, y] \in \mathcal{M}_{g-j, 1}$ carries a $\mathfrak{g}_{d}^{r}$ with ramification sequence at $y$ being at least $\left(d-r-\alpha_{r}, \ldots, d-r-\alpha_{0}\right)$, is that $\sigma_{(0,1, \ldots, 1)}^{g-j} \cdot \sigma_{\left(d-r-\alpha_{r}, \ldots, d-r-\alpha_{0}\right)} \neq 0 \in H^{*}(\mathbf{G}(r, d))$. Using the Littlewood-Richardson rule, we find that this implies that $\alpha_{r} \leq r s+s-j$. A similar reasoning can be used for $C$. Degenerating $C$ to a stable curve consisting of a rational spine and $j$ elliptic tails, we obtain that if there exists a point $y \in C$ and a $\mathfrak{g}_{d}^{r}$ with ramification sequence $\left(\alpha_{0}, \ldots, \alpha_{r}\right)$ at $y$, then either $y$ specializes to a point on the rational spine in which case we find the condition $\sigma_{(0,1, \ldots, 1)}^{j} \cdot \sigma_{\left(\alpha_{0}, \ldots, \alpha_{r}\right)} \neq 0 \in H^{*}(\mathbf{G}(r, d))$ which implies that $\alpha_{0} \geq r s-j$, or else, $y$ specializes to a point on one of the elliptic tails in which case we find that there must exist two indices $0 \leq e<f \leq r$ with $\alpha_{e} \geq \alpha_{e-1}+1$ and $\alpha_{f} \geq \alpha_{f-1}+1$, such that $\sigma_{(0,1, \ldots, 1)}^{j-1} \cdot \sigma_{\left(\alpha_{0}+1, \alpha_{e-1}+1, \alpha_{e}, \alpha_{e+1}+1, \ldots, \alpha_{f-1}+1, \alpha_{f}, \alpha_{f+1}+1, \ldots, \alpha_{r}+1\right)} \neq 0$. This last condition leads to the inequality $\alpha_{0} \geq \max \{0, r s-j-1\}$.

Suppose we are in the first case, that is, $\alpha_{0} \geq r s-j$ and moreover $\rho\left(l_{C}, y\right)=$ $\rho\left(l_{D}, y\right)=0$, which is the situation which occurs for a generic choice of $y \in C$. Then $l_{C}(-(r s-j) y)=\left|L_{C}\right|$, where $L_{C} \in \operatorname{Pic}^{r+j}(C)$ with $\alpha_{r}^{L_{C}}(y) \leq s$ and $\sum_{i=0}^{r} \alpha_{i}^{L_{C}}(y)=j$, that is, $\left(L_{C}, y\right) \in X_{j, \alpha}$. If $\alpha_{0} \geq r s-j$ but now $\rho\left(l_{C}, y\right)=-1$ and $\rho\left(l_{D}, y\right) \leq 1$, then 
$\left\{\alpha_{i}^{L_{C}}(y)-(r s-j)\right\}_{i=0 \ldots r}$ must be one of the partitions from the set $\mathcal{P}_{2}$. Choosing such a partition, we have $M_{j, \beta}$ choices for the $C$-aspect, while $l_{D} \in Y_{g-j, \alpha^{L} C(y)-(r s-j)}$. Finally let us assume that we are in the case $\alpha_{0}^{l_{C}}(y)=r s-j-1$. Then necessarily $\alpha_{1}^{l_{C}}(y) \geq r s-j$, $\rho\left(l_{C}, y\right)=\rho\left(l_{D}, y\right)=0$ and $l_{C}(-(r s-j-1) y) \in U_{j, \beta}$, where $\beta_{i}:=\alpha_{i}^{l_{C}}(y)-(r s-j-1)$ for $i=0 \ldots r$. This accounts for the third sum in $\sigma^{*}\left(C^{j}\right)$. Arguing along the lines of [EH2], Lemma 3.4, $\widetilde{\mathfrak{G}}_{d}^{r}$ is smooth along $\sigma^{*}\left(C^{j}\right)$ and since all limit $\mathfrak{g}_{d}^{r}$ described in this proof are smoothable, we obtain that the claimed formula holds at the level of 1-cycles (including multiplicities).

The next corollary shows that "ghost" components of $\widetilde{\mathfrak{G}}_{d}^{r}$ having dimension > $3 g-3$, do not matter in the calculation of $\left[\sigma_{*}\left(\mathcal{G}_{i, 2}-\mathcal{H}_{i, 2}\right)\right]$.

Corollary 2.5. In the case $\rho(g, r, d)=0$, every irreducible component of $\mathcal{Z}$ of $\widetilde{\mathfrak{G}}_{d}^{r}$ such that $\operatorname{dim}(\mathcal{Z}) \geq 3 g-2$ has the property that $\operatorname{dim} \sigma(\mathcal{Z}) \leq 3 g-5$.

Proof. For a general $[C] \in \mathcal{M}_{g}$, the scheme $W_{d}^{r}(C)$ is reduced and 0-dimensional, thus every component of $\widetilde{\mathfrak{G}}_{d}^{r}$ mapping dominantly onto $\widetilde{\mathcal{M}}_{g}$ must have dimension $3 g-3$. Suppose that $\mathcal{Z}$ is a component of dimension at least $3 g-2$ such that $\sigma(\mathcal{Z})$ is a divisor on $\widetilde{\mathcal{M}}_{g}$. Then for any $[C] \in \sigma(\mathcal{Z})$ we have that $\operatorname{dim} \sigma^{-1}([C]) \geq 2$. Since this property does not hold along any of the curves $\sigma^{*}\left(C^{j}\right)$ for any $0 \leq j \leq[\mathrm{g} / 2]$ (see Proposition 2.4), it follows that $\sigma(\mathcal{Z})$ is disjoint from the test curves $C^{j} \subset \overline{\mathcal{M}}_{g}$ for all $j \geq 0$. This implies then that $[\sigma(\mathcal{Z})]=0 \in \operatorname{Pic}\left(\widetilde{\mathcal{M}}_{g}\right)$, hence $\sigma(\mathcal{Z})=0$ (use that the Satake compactification of $\mathcal{M}_{g}$ has boundary of codimension 2 ). This is a contradiction.

Let $C$ be a Brill-Noether general curve of genus $g-1$ (recall that $g=r s+s$ and $d=r s+r)$. Then $\operatorname{dim} W_{d}^{r}(C)=r$ and it is easy to see that $C$ carries no $\mathfrak{g}_{d-1}^{r}$ 's or $\mathfrak{g}_{d}^{r+1}$ 's, hence every $L \in W_{d}^{r}(C)$ corresponds to a complete and base point free linear series. We denote by $\mathcal{L}$ the Poincaré bundle on $C \times \operatorname{Pic}^{d}(C)$ and by $\pi_{1}: C \times \operatorname{Pic}^{d}(C) \rightarrow C$ and $\pi_{2}: C \times \operatorname{Pic}^{d}(C) \rightarrow \operatorname{Pic}^{d}(C)$ the projections. We define the cohomology class $\eta=\pi_{1}^{*}([$ point $]) \in H^{2}\left(C \times \operatorname{Pic}^{d}(C)\right)$, and if $\delta_{1}, \ldots, \delta_{2 g} \in H^{1}(C, \mathbb{Z}) \cong H^{1}\left(\operatorname{Pic}^{d}(C), \mathbb{Z}\right)$ is a symplectic basis, then we set

$$
\gamma:=-\sum_{\alpha=1}^{g}\left(\pi_{1}^{*}\left(\delta_{\alpha}\right) \pi_{2}^{*}\left(\delta_{g+\alpha}\right)-\pi_{1}^{*}\left(\delta_{g+\alpha}\right) \pi_{2}^{*}\left(\delta_{\alpha}\right)\right) .
$$

We have the formula (cf. ACGH], p. 335) $c_{1}(\mathcal{L})=d \eta+\gamma$, corresponding to the Hodge decomposition of $c_{1}(\mathcal{L})$. We also record that $\gamma^{3}=\gamma \eta=0, \eta^{2}=0$ and $\gamma^{2}=-2 \eta \pi_{2}^{*}(\theta)$. On $W_{d}^{r}(C)$ we have the tautological rank $r+1$ vector bundle $\mathcal{E}:=\left(\pi_{2}\right)_{*}\left(\mathcal{L}_{\mid C \times W_{d}^{r}(C)}\right)$. The Chern numbers of $\mathcal{E}$ can be computed using the Harris-Tu formula (cf. [HT]): if we write $\sum_{i=0}^{r} c_{i}\left(\mathcal{E}^{\vee}\right)=\left(1+x_{1}\right) \cdots\left(1+x_{r+1}\right)$, then for every class $\zeta \in H^{*}\left(\operatorname{Pic}^{d}(C), \mathbb{Z}\right)$ one has the formula 1

$$
x_{1}^{i_{1}} \cdots x_{r+1}^{i_{r+1}} \zeta=\operatorname{det}\left(\frac{\theta^{g-1+r-d+i_{j}-j+l}}{\left(g-1+r-d+i_{j}-j+l\right) !}\right)_{1 \leq j, l \leq r+1} \zeta .
$$

\footnotetext{
${ }^{1}$ There is a confusing sign error in the formula (1.4) in $[\mathrm{HT}]$ : the formula is correct as it is appears in $[\mathrm{HT}]$, if the $x_{j}$ 's denote the Chern roots of the dual of the kernel bundle.
} 
If we use the expression of the Vandermonde determinant, we get the formula

$$
\operatorname{det}\left(\frac{1}{\left(a_{j}+l-1\right) !}\right)_{1 \leq j, l \leq r+1}=\frac{\Pi_{j>l}\left(a_{l}-a_{j}\right)}{\Pi_{j=1}^{r+1}\left(a_{j}+r\right) !} .
$$

By repeatedly applying this formula we compute all the intersection numbers on $W_{d}^{r}(C)$ which we shall need:

Lemma 2.6. If $c_{i}:=c_{i}\left(\mathcal{E}^{\vee}\right)$ we have the following identities in $H^{*}\left(W_{d}^{r}(C), \mathbb{Z}\right)$ :

(1) $c_{r-1} \theta=\frac{r(s+1)}{2} c_{r}$.

(2) $c_{r-2} \theta^{2}=\frac{r(r-1)(s+1)(s+2)}{6} c_{r}$.

(3) $c_{r-2} c_{1} \theta=\frac{r(s+1)}{2}\left(1+\frac{(r-2)(r+2)(s+2)}{3(s+r+1)}\right) c_{r}$.

(4) $c_{r-1} c_{1}=\left(1+\frac{(r-1)(r+2)(s+1)}{2(s+r+1)}\right) c_{r}$.

(5) $c_{r}=\frac{1 ! 2 ! \cdots(r-1) !(r+1) !}{(s-1) !(s+1) !(s+2) ! \cdots(s+r) !} \theta^{g-1}$.

We point out that the constant $c_{r}$ equals the number of linear series $\mathfrak{g}_{d}^{r}$ on a general curve of genus $g$ (note that $\rho(g, r, d)=0$ ). In Section 3 we shall use the following result:

Lemma 2.7. If $[C] \in \mathcal{M}_{g-1}$, then one has the following identity in $H^{*}\left(W_{d}^{r}(C), \mathbb{Z}\right)$ :

$$
c_{1}\left(R^{1} \pi_{2 *}\left(\mathcal{L}_{\mid C \times W_{d}^{r}(C)}\right)\right)=\theta-c_{1}\left(\mathcal{E}^{\vee}\right) .
$$

Proof. Let us recall how one can obtain a determinantal structure on $W_{d}^{r}(C)$. Once we fix a divisor $D \in C_{e}$ of degree $e>>0, W_{d}^{r}(C)$ is the degeneracy locus of rank $d+e-g+1-r$ of the vector bundle map $\left(\pi_{2}\right)_{*}\left(\mathcal{L} \otimes \mathcal{O}\left(\pi_{1}^{*} D\right)\right) \rightarrow\left(\pi_{2}\right)_{*}\left(\mathcal{L} \otimes \mathcal{O}\left(\pi_{1}^{*} D\right)_{\mid \pi_{1}^{*}(D)}\right)$. Consequently, we have an exact sequence of vector bundles over $W_{d}^{r}(C)$ :

$0 \longrightarrow \mathcal{E} \longrightarrow\left(\pi_{2}\right)_{*}\left(\mathcal{L} \otimes \mathcal{O}\left(\pi_{1}^{*} D\right)\right) \longrightarrow\left(\pi_{2}\right)_{*}\left(\mathcal{L} \otimes \mathcal{O}\left(\pi_{1}^{*} D\right)_{\mid \pi_{1}^{*} D}\right) \longrightarrow R^{1} \pi_{2 *}\left(\mathcal{L}_{\mid C \times W_{d}^{r}(C)}\right) \longrightarrow 0$, from which the claim follows by using that $\left(\pi_{2}\right)_{*}\left(\mathcal{L} \otimes \mathcal{O}\left(\pi^{*} D\right)_{\mid \pi_{1}^{*} D}\right)$ is numerically trivial while $c_{t}\left(\left(\pi_{2}\right)_{*}\left(\mathcal{L} \otimes \mathcal{O}\left(\pi_{1}^{*} D\right)\right)\right)=e^{-\theta}($ cf. [EH3] or [ACGH] $)$.

For integers $0 \leq a \leq r$ and $b \geq 2$ we shall define vector bundles $\mathcal{G}_{a, b}$ and $\mathcal{H}_{a, b}$ over $\sigma^{-1}\left(\mathcal{M}_{g}^{0} \cup \Delta_{0}^{0} \cup \Delta_{1}^{0}\right) \subset \widetilde{\mathfrak{G}}_{d}^{r}$ which over the locus corresponding to smooth curves have fibres

$$
\mathcal{G}_{a, b}(C, L)=H^{0}\left(C, \wedge^{a} M_{L} \otimes L^{\otimes b}\right) \text { and } \mathcal{H}_{a, b}(C, L)=H^{0}\left(\mathbf{P}^{r}, \wedge^{a} M_{\mathbf{P}^{r}}(b)\right)
$$

for each $(C, L) \in \mathfrak{G}_{d}^{r}$ giving a map $C \stackrel{|L|}{\rightarrow} \mathbf{P}^{r}$. Clearly $\mathcal{G}_{i, 2 \mid \mathfrak{G}_{d}^{r}}=\mathcal{B}$ and $\mathcal{H}_{i, 2 \mid \mathfrak{G}_{d}^{r}}=\mathcal{A}$, where $\mathcal{A}$ and $\mathcal{B}$ are the vector bundles introduced in Theorem 2.1. Partially extending these bundles over the boundary of $\widetilde{\mathfrak{G}}_{d}^{r}$ will enable us to compute the $\lambda, \delta_{0}$ and $\delta_{1}$ coefficients of $\overline{\mathcal{Z}}_{g, i}$.

Proposition 2.8. For each $b \geq 2$ there exists a vector bundle $\mathcal{G}_{0, b}$ over $\sigma^{-1}\left(\mathcal{M}_{g}^{0} \cup \Delta_{0}^{0} \cup \Delta_{1}^{0}\right) \subset$ $\widetilde{\mathfrak{G}}_{d}^{r}$ having rank bd $+1-g$ whose fibres admit the following description:

- For $(C, L) \in \mathfrak{G}_{d^{\prime}}^{r}$ we have that $\mathcal{G}_{0, b}(C, L)=H^{0}\left(C, L^{\otimes b}\right)$. 
- For $t=\left(C \cup_{y} E, L\right) \in \sigma^{-1}\left(\Delta_{1}^{0}\right)$, where $L \in W_{d}^{r}(C)$ has a cusp at $y \in C$, we have that

$$
\mathcal{G}_{0, b}(t)=H^{0}\left(C, L^{\otimes b}(-2 y)\right)+\mathbb{C} \cdot u^{b} \subset H^{0}\left(C, L^{\otimes b}\right),
$$

where $u \in H^{0}(C, L)$ is any section such that $\operatorname{ord}_{y}(u)=0$.

- For $t=(C / y \sim q, L) \in \sigma^{-1}\left(\Delta_{0}^{0}\right)$, where $q, y \in C$ and $L \in W_{d}^{r}(C)$ is such that $h^{0}(C, L(-y-q))=h^{0}(L)-1$, we have that

$$
\mathcal{G}_{0, b}(t)=H^{0}\left(C, L^{\otimes b}(-y-q)\right) \oplus \mathbb{C} \cdot u^{b} \subset H^{0}\left(C, L^{\otimes b}\right),
$$

where $u \in H^{0}(C, L)$ is a section such that $\operatorname{ord}_{y}(u)=\operatorname{ord}_{q}(u)=0$.

Proof. Very similar to Proposition 3.9 in [F1].

Having defined the vector bundles $\mathcal{G}_{0, b}$ we now define inductively all vector bundles $\mathcal{G}_{a, b}$ by the exact sequence

$$
0 \longrightarrow \mathcal{G}_{a, b} \longrightarrow \wedge^{a} \mathcal{G}_{0,1} \otimes \mathcal{G}_{0, b} \stackrel{d_{a, b}}{\longrightarrow} \mathcal{G}_{a-1, b+1} \longrightarrow 0 .
$$

To define $\mathcal{H}_{a, b}$ is even easier. We set $\mathcal{H}_{0, b}:=\operatorname{Sym}^{b} \mathcal{G}_{0,1}$ for all $b \geq 1$ and we define $\mathcal{H}_{a, b}$ inductively via the exact sequence

$$
0 \longrightarrow \mathcal{H}_{a, b} \longrightarrow \wedge^{a} \mathcal{H}_{0,1} \otimes \operatorname{Sym}^{b} \mathcal{H}_{0,1} \longrightarrow \mathcal{H}_{a-1, b+1} \longrightarrow 0 .
$$

The surjectivity of the right map in (5) is obvious, whereas to prove that $d_{a, b}$ is surjective, one argues like in [F1], Proposition 3.10. There is a natural vector bundle morphism $\phi_{a, b}: \mathcal{H}_{a, b} \rightarrow \mathcal{G}_{a, b}$. Moreover $\operatorname{rank}\left(\mathcal{H}_{i, 2}\right)=\operatorname{rank}\left(\mathcal{G}_{i, 2}\right)$ and the degeneracy locus of $\phi_{i, 2}$ is the codimension one compactification of $\mathcal{Z}_{g, i}$ over $\mathcal{M}_{g}^{0} \cup \Delta_{0}^{0} \cup \Delta_{1}^{0}$.

We prove a technical result we shall use later for extending the bundles $\mathcal{G}_{0, b}$ with $b \geq 2$ over the boundary of $\mathfrak{G}_{d}^{r}$. It can be interpreted as saying that on a suitably general curve, the ramification points of a linear series are distinct from those of its higher order powers.

Proposition 2.9. Fix integers $s \geq 2, r \geq 2 s$ and a partition $0 \leq \beta_{0} \leq \beta_{1} \leq \cdots \leq \beta_{r} \leq s$ such that $\sum_{i=0}^{r} \beta_{i}=\gamma$. Let $(D, y)$ be a general pointed curve of genus $\gamma \geq 3$ s. Then for every line bundle $L_{D} \in \operatorname{Pic}^{\gamma+r}(D)$ satisfying the conditions $\alpha_{i}^{L_{D}}(y)=\beta_{i}$ for $0 \leq i \leq r$, we have that

$$
H^{0}\left(D, K_{D} \otimes L_{D}^{\otimes(-2)} \otimes \mathcal{O}_{D}(a y)\right)=0, \text { for all } a \leq 2(r+s) .
$$

Proof. Clearly it suffices to prove the theorem in the case $a=2(r+s)$. We degenerate $(D, y)$ to a stable curve $E_{0} \cup \ldots \cup E_{\gamma-1}$, consisting of a string of elliptic curves such that $E_{i-1} \cap E_{i}=\left\{p_{i}\right\}$ for $1 \leq i \leq \gamma-1$. Moreover, we assume that $y=p_{0}$ specializes to a point lying on $E_{0}$ and that the differences $p_{i}-p_{i-1} \in \operatorname{Pic}^{0}\left(E_{i-1}\right)$ are not torsion for all $1 \leq i \leq \gamma-1$. We assume by contradiction that $H^{0}\left(D, K_{D} \otimes L_{D}^{\otimes(-2)} \otimes \mathcal{O}_{D}(2(r+s) y)\right) \neq 0$ for some $L_{D} \in \operatorname{Pic}^{\gamma+r}(D)$ and denote by $L_{E_{i}} \in \operatorname{Pic}^{\gamma+r}\left(E_{i}\right)$ the $E_{i}$-aspect of the induced limit linear series $\mathfrak{g}_{r+\gamma}^{r}$ on $\cup_{i=0}^{\gamma-1} E_{i}$ satisfying the ramification conditions $\alpha_{t}^{L_{E_{0}}}\left(p_{0}\right)=\alpha_{t}$ for $0 \leq t \leq r$. Fix an integer $1 \leq i \leq \gamma-1$. By the additivity of the Brill-Noether number, we have that $\rho\left(L_{E_{i-1}}, p_{i}, p_{i-1}\right)=0$ and there exists an integer $0 \leq k \leq r$ such that $\alpha_{t}^{L_{E_{i}}}\left(p_{i}\right)=\alpha_{t}^{L_{E_{i-1}}}\left(p_{i-1}\right)+1$ for $t \neq k$ while $\alpha_{k}^{L_{E_{i}}}\left(p_{i}\right)=\alpha_{k}^{L_{E_{i-1}}}\left(p_{i-1}\right)$. In particular,

$$
L_{E_{i-1}}=\mathcal{O}_{E_{i-1}}\left(\left(\alpha_{k}^{L_{E_{i-1}}}\left(p_{i-1}\right)+k\right) \cdot p_{i-1}+\left(\gamma+r-k-\alpha_{k}^{L_{E_{i-1}}}\left(p_{i-1}\right)\right) \cdot p_{i}\right),
$$


that is $L_{E_{i-1}}$ corresponds to a divisor supported only at the points $p_{i-1}$ and $p_{i}$. Our assumption implies that for all $0 \leq i \leq \gamma-1$ there exist sections

$$
\left.0 \neq \rho_{i} \in H^{0}\left(E_{i}, \mathcal{O}_{E_{i}}\left(2(r+s+i) p_{i}+2(\gamma-i-1) p_{i+1}\right) \otimes L_{E_{i}}^{\otimes(-2)}\right)\right)
$$

satisfying the compatibility conditions

$$
\operatorname{ord}_{p_{i}}\left(\rho_{i}\right) \geq \operatorname{ord}_{p_{i-1}}\left(\rho_{i-1}\right) \text { and } \operatorname{ord}_{p_{i}}\left(\rho_{i-1}\right)+\operatorname{ord}_{p_{i}}\left(\rho_{i}\right)=2 s-2 .
$$

We reach a contradiction once we show that

$$
\operatorname{ord}_{p_{\gamma-1}}\left(\rho_{\gamma-1}\right)>2 s-2=\operatorname{deg}\left(\mathcal{O}_{E_{\gamma-1}}\left(2(r+s+\gamma-1) p_{\gamma-1} \otimes L_{E_{\gamma-1}}^{\otimes(-2)}\right)\right)
$$

which gives that $\rho_{\gamma-1}=0$. Assume now that $\operatorname{ord}_{p_{i+1}}\left(\rho_{i+1}\right)=\operatorname{ord}_{p_{i}}\left(\rho_{i}\right)$ for some $0 \leq$ $i \leq \gamma-2$. Then $\operatorname{ord}_{p_{i}}\left(\rho_{i}\right)+\operatorname{ord}_{p_{i+1}}\left(\rho_{i}\right)=2 s-2$, hence the section $\rho_{i}$ vanishes only at $p_{i}$ and $p_{i+1} \in E_{i}$ and $\operatorname{ord}_{p_{i}}\left(\rho_{i}\right)=2 b$ for some integer $b \geq 0$. We must have that $L_{E_{i}}=$ $\mathcal{O}_{E_{i}}\left((r+s+i-b) \cdot p_{i}+(\gamma-i-s-b) \cdot p_{i+1}\right)$ (we use that $p_{i+1}-p_{i} \in \operatorname{Pic}^{0}\left(E_{i}\right)$ is not torsion). In particular $r+s+i-b$ is one entry in the vanishing sequence $a^{L_{E_{i}}}\left(p_{i}\right)$ and the vanishing sequence $a^{L_{E_{i+1}}}\left(p_{i+1}\right)$ is obtained from $a^{L_{i}}\left(p_{i}\right)$ by raising all entries by 1 , except for $r+s+i-b$ which remains unmodified. Obviously then, the number $r+s+i+1-b$ cannot appear in the vanishing sequence $a^{L_{E_{i+1}}}\left(p_{i+1}\right)$. But this implies that $\operatorname{ord}_{p_{i+2}}\left(\rho_{i+2}\right) \geq$ $\operatorname{ord}_{p_{i+1}}\left(\rho_{i+1}\right)+1$. This argument shows that as we trace the non-decreasing sequence of vanishing orders $\left\{\operatorname{ord}_{p_{i}}\left(\rho_{i}\right)\right\}_{i=0}^{\gamma-1}$ along any group of 3 consecutive components $E_{i-1}, E_{i}$ and $E_{i+1}$, we will find at least 2 along which $\operatorname{ord}_{p_{i}}\left(\rho_{i}\right)$ jumps. Since $\gamma \geq 3 s$, we find that $\operatorname{ord}_{p_{\gamma-1}}\left(\rho_{\gamma-1}\right)>2 s-2$ and this brings about a contradiction.

Next we extend $\mathcal{G}_{a, b}$ and $\mathcal{H}_{a, b}$ over the divisors $\sigma^{-1}\left(\Delta_{j}^{0}\right)$ for $[g / 2] \leq j \leq g-2$.

Proposition 2.10. (1) For $g=r s+s, d=r s+r$ and $b \geq 1$, there exists a vector bundle $\mathcal{G}_{0, b}$ defined over $\widetilde{\mathfrak{G}}_{d^{\prime}}^{r}$ extending the already constructed vector bundle $\mathcal{G}_{0, b}$ over $\sigma^{-1}\left(\mathcal{M}_{g}^{0} \cup \Delta_{0}^{0} \cup \Delta_{1}^{0}\right)$ and such that if $t=\left(C \cup_{y} D, l_{C}, l_{D}\right) \in \sigma^{-1}\left(\Delta_{j}^{0}\right)$, where $g(C)=j \geq[s(r+1) / 2], g(D)=g-j \geq 2$ and $\left(l_{C}, l_{D}\right)$ is a limit $\mathfrak{g}_{d}^{r}$ on $C \cup_{y} D$, then

$$
\mathcal{G}_{0, b}(t)=H^{0}\left(C \cup_{y} D, L^{\otimes b}\right),
$$

with

$$
\begin{aligned}
& L:=\left(L_{C}=l_{C}(-(r s-j) y), L_{D}=l_{D}(-(j+r) y)\right) \in \operatorname{Pic}^{j+r}(C) \times \operatorname{Pic}^{r s-j}(D), \\
& \text { in the case } s(r+1) / 2 \leq j \leq s(r-1)=g-2 s, \text { and } \\
& L:=\left(L_{C}=l_{C}(-(g-j+r) y), L_{D}=l_{D}(-(j-s) y)\right) \in \operatorname{Pic}^{j-s}(C) \times \operatorname{Pic}^{r s+s-j+r}(D),
\end{aligned}
$$

in the case $s(r-1)<j \leq s(r+1)-2$.

(2) For each $0 \leq a \leq r, b \geq 1$ there exists a vector bundle $\mathcal{H}_{a, b}$ over $\widetilde{\mathfrak{G}}_{d}^{r}$ restricting to the already defined vector bundle $\mathcal{H}_{a, b}$ over $\sigma^{-1}\left(\mathcal{M}_{g}^{0} \cup \Delta_{0}^{0} \cup \Delta_{1}^{0}\right)$, such that $\mathcal{H}_{0, b}=$ $\operatorname{Sym}^{b}\left(\mathcal{G}_{0,1}\right)$ for all $b \geq 1$ and which also has the property that the exact sequences (5) remain exact over $\widetilde{\mathfrak{G}}_{d}^{r}$.

(3) For each $0 \leq a \leq r, b \geq 1$, there exists a torsion free sheaf $\mathcal{G}_{a, b}$ over $\widetilde{\mathfrak{G}}_{d}^{r}$ that restricts to the vector bundle $\mathcal{G}_{a, b}$ over $\sigma^{-1}\left(\mathcal{M}_{g}^{0} \cup \Delta_{0}^{0} \cup \Delta_{1}^{0}\right)$, which for $a=0$ agrees with the vector bundle $\mathcal{G}_{0, b}$ defined above, and which has the property that the vector bundle morphisms 
$\phi_{a, b}$ defined over $\sigma^{-1}\left(\mathcal{M}_{g}^{0} \cup \Delta_{0}^{0} \cup \Delta_{1}^{0}\right)$ extend to morphisms $\phi_{a, b}: \mathcal{H}_{a, b} \rightarrow \mathcal{G}_{a, b}$ over $\widetilde{\mathfrak{G}}_{d}^{r}$.

Proof. We start with an arbitrary point $t=\left(C \cup_{y} D, l_{C}, l_{D}\right) \in \sigma^{*}\left(C^{j}\right)$ where we assume first that $[g / 2] \leq j \leq r s-s$. We set $L_{C}:=l_{C}(-(r s-j) y) \in \operatorname{Pic}^{r+j}(C)$ and $L_{D}:=l_{D}(-(r+$ $j) y) \in \operatorname{Pic}^{r s-j}(D)$. If $L=\left(L_{C}, L_{D}\right) \in \operatorname{Pic}^{r s+r}\left(C \cup_{y} D\right)$, the essential observation is that because $[C] \in \mathcal{M}_{j}$ and $[D, y] \in \mathcal{M}_{g-j, 1}$ are Brill-Noether general, we always have that $r s+r-1 \leq a_{0}^{l_{C}}(y)+a_{r}^{l_{D}}(y) \leq r s+r$, hence $h^{0}\left(L_{C}\right) \geq r, h^{0}\left(L_{D}\right) \leq 1$ and $h^{0}\left(C \cup_{y} D, L\right)=$ $r+1$ (see Proposition 2.4). If $p: \mathcal{Y}:=\widetilde{\mathfrak{G}}_{d}^{r} \times \widetilde{\mathcal{M}}_{g} \widetilde{\mathcal{M}}_{g, 1} \rightarrow \widetilde{\mathfrak{G}}_{d}^{r}$ is the universal curve over $\widetilde{\mathfrak{G}}_{d}^{r}$, we denote by $\mathcal{P}$ the Poincaré bundle of degree $d=r s+r$ having the property that for each $[g / 2] \leq j \leq g-2, \mathcal{P}_{\mid p^{-1}\left(\sigma^{-1}\left(\Delta_{j}^{0}\right)\right)}$ parametrizes line bundles of bidegree $(r+j, r s-j)$ on curves of type $C \cup_{y} D$ where $g(C)=j$ and $g(D)=g-j$ (Note that since the divisors $\sigma^{-1}\left(\Delta_{i}^{0}\right)$ and $\sigma^{-1}\left(\Delta_{j}^{0}\right)$ are disjoint for $[g / 2] \leq i<j \leq g-2$, the construction can be carried out over a fixed divisor $\Delta_{j}^{0}$ at a time). Since $h^{0}\left(p^{-1}(t), \mathcal{P}_{\mid p^{-1}(t)}\right)=h^{0}\left(C \cup_{y} D, L\right)=$ $r+1$ for each $t \in \widetilde{\mathfrak{G}}_{d}^{r}$, by Grauert's Theorem, $\mathcal{G}_{0,1}:=p_{*}(\mathcal{P})$ is a locally free sheaf which satisfies our first requirement. For $b \geq 2$ we define $\mathcal{G}_{0, b}=p_{*}\left(\mathcal{P}^{\otimes b}\right)$. Based on degree considerations we have that $H^{1}\left(L_{C}^{\otimes b}\right)=H^{1}\left(L_{C}^{\otimes b} \otimes \mathcal{O}_{C}(-y)\right)=0$. Using Proposition 2.9 we see that $H^{1}\left(L_{D}^{\otimes b}\right)=0$, hence $h^{0}\left(C \cup D, L^{\otimes b}\right)=h^{0}\left(L_{C}^{\otimes b}\right)+h^{0}\left(L_{D}^{\otimes b}\right)-1=b d+1-g$. Grauert's Theorem implies that $\mathcal{G}_{0, b}$ is locally free for all $b$.

In the remaining case when $r s-s+1 \leq j \leq g-2$, that is, $2 \leq g(D) \leq s-1$, we define $L_{C}:=l_{C}(-(r s+s+r-j) y) \in \operatorname{Pic}^{j-s}(C)$ and $L_{D}:=l_{D}(-(j-s) y) \in$ $\operatorname{Pic}^{r s+s-j+r}(D)$. Then $h^{0}\left(L_{C}\right) \leq 1, h^{0}\left(L_{D}\right) \geq r$ and $h^{0}\left(C \cup_{y} D, L\right)=r+1$. Proposition 2.9 gives again that $h^{1}\left(L_{C}^{\otimes b}\right)=0$ for all $b \geq 2$, hence $h^{0}\left(C \cup D, L^{\otimes b}\right)=b d+1-g$. This time, we denote by $\mathcal{P}$ the Poincaré bundle parametrizing line bundles of bidegree $(j-s, r s+s+r-j)$ on curves of type $C \cup_{y} D$ and then $\mathcal{G}_{0, b}:=p_{*}\left(\mathcal{P}^{\otimes b}\right)$ is locally free in this case too because of Grauert's Theorem.

To define $\mathcal{G}_{a, b}$ for $a \geq 1$, we introduce the sheaf $\mathcal{M}:=\operatorname{Ker}\left\{p^{*}\left(p_{*}(\mathcal{P})\right) \rightarrow \mathcal{P}\right\}$ and then we set $\mathcal{G}_{a, b}:=p_{*}\left(\wedge^{a} \mathcal{M} \otimes \mathcal{P}^{\otimes b}\right)$. The morphism $\phi_{0, b}$ is simply the natural map $\operatorname{Sym}^{b} p_{*}(\mathcal{P}) \rightarrow p_{*}\left(\mathcal{P}^{\otimes b}\right)$, and to define these maps for $a \geq 1$ we use that the vector bundles $\mathcal{H}_{a, b}$ fit into exact sequences of type (5) and then proceed inductively.

We now determine the class of the curves $X$ and $Y$ defined in Proposition 2.3,

Proposition 2.11. Let $C$ be a Brill-Noether general curve of genus $g-1$ and $q \in C$ a general point. We denote by $\pi_{2}: C \times W_{d}^{r}(C) \rightarrow W_{d}^{r}(C)$ the projection and set $c_{i}:=\left(\pi_{2}\right)^{*}\left(c_{i}\left(\mathcal{E}^{\vee}\right)\right)$.

(1) The class of the curve $X=\left\{(y, L) \in C \times W_{d}^{r}(C): h^{0}(C, L(-2 y)) \geq r\right\}$ is given by

$$
[X]=c_{r}+c_{r-1}(2 \gamma+(2 d+2 g-4) \eta)-6 c_{r-2} \eta \theta .
$$

(2) The class of the curve $Y=\left\{(y, L) \in C \times W_{d}^{r}(C): h^{0}(C, L(-y-q)) \geq r\right\}$ is given by

$$
[Y]=c_{r}+c_{r-1}(\gamma+(d-1) \eta)-2 c_{r-2} \eta \theta .
$$

Proof. We realize both $X$ and $Y$ as degeneracy loci over $C \times W_{d}^{r}(C)$ and compute their classes using the Thom-Porteous formula. For each $(y, L) \in C \times W_{d}^{r}(C)$ we have a 
natural map

$$
H^{0}\left(C, L_{\mid 2 y}\right)^{\vee} \rightarrow H^{0}(C, L)^{\vee}
$$

which globalizes to a vector bundle map $\zeta: J_{1}(\mathcal{L})^{\vee} \rightarrow\left(\pi_{2}\right)^{*}\left(\mathcal{E}^{\vee}\right)$. Clearly $X=Z_{1}(\zeta)$, hence

$$
[X]=\left[\frac{c_{t}\left(\pi_{2}^{*}\left(\mathcal{E}^{\vee}\right)\right)}{c_{t}\left(J_{1}(\mathcal{L})^{\vee}\right)}\right]_{r}
$$

¿From the exact sequence

$$
0 \longrightarrow \pi_{1}^{*}\left(K_{C}\right) \otimes \mathcal{L} \rightarrow J_{1}(\mathcal{L}) \rightarrow \mathcal{L} \rightarrow 0
$$

we obtain that $c_{t}\left(J_{1}\left(\mathcal{L}^{\vee}\right)\right)^{-1}=1+2 \gamma+2 d \eta+(2 g-4) \eta-6 \eta \theta$, which quickly leads to the desired expression for $[X]$. The calculation of $[Y]$ is entirely similar and we skip it.

We also need the following intersection theoretic result:

Lemma 2.12. For each $j \geq 2$ we have the following formulas:

(1) $c_{1}\left(\mathcal{G}_{0, j \mid X}\right)=-j^{2} \theta-(2 g-4) \eta-j(d \eta+\gamma)$.

(2) $c_{1}\left(\mathcal{G}_{0, j \mid Y}\right)=-j^{2} \theta+\eta$.

Proof. We observe that for all $j \geq 2, H^{1}\left(L^{\otimes j}\right)=0$, hence $\left(\pi_{2}\right)_{*}\left(\mathcal{L}^{\otimes j}\right)$ is a vector bundle over $\operatorname{Pic}^{d}(C)$. Riemann-Roch applied to the map $\pi_{2}: C \times \operatorname{Pic}^{d}(C) \rightarrow \operatorname{Pic}^{d}(C)$ yields $c_{1}\left(\left(\pi_{2}\right)_{*}\left(\mathcal{L}^{\otimes j}\right)\right)=-j^{2} \theta$. If we denote by $u, v: C \times C \times \operatorname{Pic}^{d}(C) \rightarrow C \times \operatorname{Pic}^{d}(C)$ the two projections and by $\Delta \subset C \times C \times \operatorname{Pic}^{d}(C)$ the diagonal, we have the following exact sequences

$$
0 \longrightarrow u_{*}\left(v^{*}\left(\mathcal{L}^{\otimes j}\right) \otimes \mathcal{I}_{\Delta}^{2}\right)_{\mid X} \longrightarrow \mathcal{G}_{0, j \mid X} \longrightarrow \mathcal{L}_{\mid X}^{\otimes j} \longrightarrow 0
$$

and

$$
0 \longrightarrow u_{*}\left(v^{*}\left(\mathcal{L}^{\otimes j}\right) \otimes \mathcal{I}_{\Delta}^{2}\right) \longrightarrow\left(\pi_{2}\right)_{*}\left(\mathcal{L}^{\otimes j}\right) \longrightarrow J_{1}\left(\mathcal{L}^{\otimes j}\right) \longrightarrow 0
$$

(and an entirely similar situation for $\mathcal{G}_{0, j \mid Y}$ ) from which both claims follow easily.

Now we are in a position to prove Theorem 1.1:

Proof of Theorem 1.1. Since $\operatorname{codim}\left(\overline{\mathcal{M}}_{g}-\widetilde{\mathcal{M}}_{g}, \overline{\mathcal{M}}_{g}\right) \geq 2$, it makes no difference whether we compute the class $\sigma_{*}\left(\mathcal{G}_{i, 2}-\mathcal{H}_{i, 2}\right)$ on $\widetilde{\mathcal{M}}_{g}$ or on $\overline{\mathcal{M}}_{g}$ and we can write

$$
\sigma_{*}\left(\mathcal{G}_{i, 2}-\mathcal{H}_{i, 2}\right)=A \lambda-B_{0} \delta_{0}-B_{1} \delta_{1}-\cdots-B_{[g / 2]} \delta_{[g / 2]} \in \operatorname{Pic}\left(\overline{\mathcal{M}}_{g}\right),
$$

where $\lambda, \delta_{0}, \ldots, \delta_{[g / 2]}$ are the generators of $\operatorname{Pic}\left(\overline{\mathcal{M}}_{g}\right)$. We start with the following:

Claim: One has the relation $A-12 B_{0}+B_{1}=0$.

We pick a general curve $[C, q] \in \mathcal{M}_{g-1,1}$ and at the fixed point $q$ we attach to $C$ a Lefschetz pencil of plane cubics. If we denote by $R \subset \overline{\mathcal{M}}_{g}$ the resulting curve, then $R \cdot \lambda=1, R \cdot \delta_{0}=12, R \cdot \delta_{1}=-1$ and $R \cdot \delta_{j}=0$ for $j \geq 2$. The relation $A-12 B_{0}+B_{1}=0$ follows once we show that $\sigma^{*}(R) \cdot c_{1}\left(\mathcal{G}_{i, 2}-\mathcal{H}_{i, 2}\right)=0$. To achieve this we check that $\mathcal{G}_{0, b \mid \sigma^{*}(R)}$ is trivial and then use (4) and (5). We take $\left[C \cup_{q} E\right] \in \overline{\mathcal{M}}_{g}$ to be an arbitrary curve from $R$, where $E$ is an elliptic curve. The pointed curve $[C, q]$ being Brill-Noether general, limit $\mathfrak{g}_{d}^{r}$ 's on $C \cup_{q} E$ are in $1: 1$ correspondence with linear series $L \in W_{d}^{r}(C)$ having a cusp at $q$ (This is a statement independent of the $j$-invariant of $E$, in particular, it also holds for the 12 rational nodal curves in the pencil). Furthermore, 
the fibre of $\mathcal{G}_{0, b \mid \sigma^{*}\left(\Delta_{1}^{0}\right)}$ over each point from $\sigma^{*}(R)$ consists of the global sections of the genus $g-1$ aspect of the limit $\mathfrak{g}_{d}^{r}$ and the claim now follows.

Now we determine explicitly the coefficients $A, B_{0}$ and $B_{1}$. We fix a general curve $(C, q) \in \mathcal{M}_{g-1,1}$ and construct the test curves $C^{1} \subset \Delta_{1}$ and $C^{0} \subset \Delta_{0}$. Using the notation from Proposition 2.3, we write that $\sigma^{*}\left(C^{0}\right) \cdot c_{1}\left(\mathcal{G}_{i, 2}-\mathcal{H}_{i, 2}\right)=c_{1}\left(\mathcal{G}_{i, 2 \mid Y}\right)-c_{1}\left(\mathcal{H}_{i, 2 \mid Y}\right)$ and $\sigma^{*}\left(C^{1}\right) \cdot c_{1}\left(\mathcal{G}_{i, 2}-\mathcal{H}_{i, 2}\right)=c_{1}\left(\mathcal{G}_{i, 2 \mid X}\right)-c_{1}\left(\mathcal{H}_{i, 2 \mid X}\right)$ (the other component $T$ of $\sigma^{*}\left(C^{1}\right)$ does not appear because $\mathcal{G}_{0, b \mid T}$ is trivial for all $b \geq 1$ ). On the other hand

$$
C^{0} \cdot \sigma_{*}\left(c_{1}\left(\mathcal{G}_{i, 2}-\mathcal{H}_{i, 2}\right)\right)=(2 g-2) B_{0}-B_{1} \text { and } C^{1} \cdot \sigma_{*}\left(c_{1}\left(\mathcal{G}_{i, 2}-\mathcal{H}_{i, 2}\right)\right)=(2 g-4) B_{1},
$$

while we already know that $A-12 B_{0}+B_{1}=0$. Next we use the relations

$$
\begin{gathered}
c_{1}\left(\mathcal{G}_{i, 2}\right)=\sum_{l=0}^{i}(-1)^{l} c_{1}\left(\wedge^{i-l} \mathcal{G}_{0,1} \otimes \mathcal{G}_{0, l+2}\right)=\sum_{l=0}^{i}(-1)^{l}\left(\begin{array}{c}
r+1 \\
i-l
\end{array}\right) c_{1}\left(\mathcal{G}_{0, l+2}\right)+ \\
+\sum_{l=0}^{i}(-1)^{l}((l+2)(r s+r)+1-r s-s)\left(\begin{array}{c}
r \\
i-l-1
\end{array}\right) c_{1}\left(\mathcal{G}_{0,1}\right), \quad \text { and } \\
c_{1}\left(\mathcal{H}_{i, 2}\right)=\sum_{l=0}^{i}(-1)^{l} c_{1}\left(\wedge^{i-l} \mathcal{G}_{0,1} \otimes \operatorname{Sym}^{l+2} \mathcal{G}_{0,1}\right)= \\
=\sum_{l=0}^{i}(-1)^{l}\left(\left(\begin{array}{c}
r \\
i-l-1
\end{array}\right)\left(\begin{array}{c}
r+l+2 \\
l+2
\end{array}\right)+\left(\begin{array}{c}
r+1 \\
i-l
\end{array}\right)\left(\begin{array}{c}
r+l+2 \\
r+1
\end{array}\right)\right) c_{1}\left(\mathcal{G}_{0,1}\right),
\end{gathered}
$$

which when restricted to $X$ and $Y$, enable us (also using Lemma2.12), to obtain explicit expressions for $c_{1}\left(\mathcal{G}_{i, 2}-\mathcal{H}_{i, 2}\right)_{\mid X}$ and $c_{1}\left(\mathcal{G}_{i, 2}-\mathcal{H}_{i, 2}\right)_{\mid Y}$ in terms of the classes $\eta, \theta, \gamma$ and $c_{1}=\pi_{2}^{*}\left(c_{1}\left(\mathcal{E}^{\vee}\right)\right)$. Intersecting these classes with $[X]$ and $[Y]$ and using Lemma 2.6, we finally get a linear system of 3 equations in $A, B_{0}$ and $B_{1}$ which leads to the stated formulas for the first three coefficients.

Next we prove that when $i=0$, we can get a formula for the slope of $\overline{\mathcal{Z}}_{s(2 s+1), 0}$ : precisely we show that if we write $\sigma_{*}\left(c_{1}\left(\mathcal{G}_{0,2}-\mathcal{H}_{0,2}\right)\right)=A \lambda-B_{0} \delta_{0}-\cdots-B_{[g / 2]} \delta_{[g / 2]}$, then $B_{j} \geq B_{0}$ for all $j \geq 1$. In particular, $s\left(\sigma_{*}\left(c_{1}\left(\mathcal{G}_{0,2}-\mathcal{H}_{0,2}\right)\right)\right)=A / B_{0}$ which has already been computed in Theorem 1.1. We note that the proof uses in an essential way the divisor class calculation from Theorem 4.6 .

Proof of Theorem 1.4. Using the convention $B_{g-j}=B_{j}$ for $g / 2 \leq j \leq g-1$, we show that $B_{j} \geq B_{0}$ only when $s(2 s+1) / 2 \leq j \leq s(2 s-1)$. The case $2 s^{2} \leq j \leq s(2 s+1)-1$ is dealt with in a similar fashion. To compute $B_{j}$ we intersect the class $\sigma_{*}\left(c_{1}\left(\mathcal{G}_{0,2}-\mathcal{H}_{0,2}\right)\right)$ with $C^{j}$. Then we use that $\left[Y_{g-j, \beta}\right] \cdot c_{1}\left(\mathcal{G}_{0,2}-\mathcal{H}_{0,2}\right)=\left[U_{j, \gamma}\right] \cdot c_{1}\left(\mathcal{G}_{0,2}-\mathcal{H}_{0,2}\right)=0$, for all $\beta \in \mathcal{P}_{2}, \gamma \in \mathcal{P}_{3}$, to obtain that

$$
(2 j-2) B_{j}=\sigma^{*}\left(C^{j}\right) \cdot c_{1}\left(\mathcal{G}_{0,2}-\mathcal{H}_{0,2}\right)=\sum_{\left(\alpha_{0}, \ldots, \alpha_{r}\right) \in \mathcal{P}_{1}} N_{g-j, \alpha}\left(\left[X_{j, \alpha}\right] \cdot c_{1}\left(\mathcal{G}_{0,2}-\mathcal{H}_{0,2}\right)\right) .
$$

We fix a Schubert index $\left(\alpha_{0} \leq \ldots \leq \alpha_{r}\right) \in \mathcal{P}_{1}$ and denote by $\pi_{1}: X_{j, \alpha} \rightarrow C$ and $\pi_{2}: X_{j, \alpha} \rightarrow \operatorname{Pic}^{r+j}(C)$ the two projection maps. As before, $\mathcal{L}$ is the Poincare bundle on $C \times \operatorname{Pic}^{r+j}(C)$. There is an isomorphism of bundles $\mathcal{G}_{0,1 \mid X_{j, \alpha}}=\pi_{2}^{*}\left(\left(\pi_{2}\right)_{*}(\mathcal{L})\right)_{\mid X_{j, \alpha}}$ obtained by globalizing the projection isomorphism at the level of spaces of sections $H^{0}\left(C \cup_{y} D, L\right) \cong H^{0}\left(C, L_{C}\right)$ valid for each point $\left(y, L_{C}\right) \in X_{j, \alpha}$. (We recall that $L=$ 
$\left.\left(L_{C}, L_{D}\right) \in \operatorname{Pic}^{2 s+j}(C) \times \operatorname{Pic}^{2 s^{2}-j}(D)\right)$. For $b \geq 2$, we have a surjective morphism of vector bundles $\mathcal{G}_{0, b \mid X_{j, \alpha}} \rightarrow \pi_{2}^{*}\left(\left(\pi_{2}\right)_{*}\left(\mathcal{L}^{\otimes b}\right)\right)_{\mid X_{j, \alpha}}$ whose kernel is a trivial bundle along $X_{j, \alpha}$. Thus one has that $c_{1}\left(\mathcal{G}_{0, b \mid X_{j, \alpha}}\right)=-b^{2} \theta_{\mid X_{j, \alpha}}$ and $c_{1}\left(\mathcal{H}_{0,2 \mid X_{j, \alpha}}\right)=c_{1}\left(\operatorname{Sym}^{2} \mathcal{G}_{0,1 \mid X_{j, \alpha}}\right)=$ $-(2 s+2) \theta_{\mid X_{j, \alpha}}$, therefore

$$
(2 j-2) B_{j}=(2 s-2) \sum_{\left(\alpha_{0}, \ldots, \alpha_{r}\right) \in \mathcal{P}_{1}} N_{g-j, \alpha}\left(\left[X_{j, \alpha}\right] \cdot \theta\right) .
$$

The class of the curve $X_{j, \alpha}$ can be computed using the generalized Giambelli formula (cf. [FuPr], pg. 15-17) as follows: If $J_{\alpha_{r}+r-1}(\mathcal{L}) \rightarrow \cdots \rightarrow J_{\alpha_{i}+i-1}(\mathcal{L}) \rightarrow \cdots \rightarrow J_{\alpha_{0}-1}(\mathcal{L})$ is the flag of jet bundles corresponding to the ramification sequence $\left(\alpha_{0}, \ldots, \alpha_{r}\right)$, then

$X_{j, \alpha}=\left\{(y, L) \in C \times \operatorname{Pic}^{r+j}(C): \operatorname{rk}\left\{\pi_{2}^{*}\left(\left(\pi_{2}\right)_{*}(\mathcal{L})\right)(y, L) \rightarrow J_{\alpha_{i}+i-1}(\mathcal{L})(y, L)\right\} \leq i\right.$ for all $\left.i\right\}$ and then $\left[X_{j, \alpha}\right]$ is given by the determinant of the $\left(\alpha_{r} \times \alpha_{r}\right)$-matrix having entries

$$
a_{i k}=c_{r+1-l+k-i}\left(\frac{\pi_{2}^{*}\left(\left(\pi_{2}\right)_{*}(\mathcal{L})\right)}{J_{\alpha_{l}+l-1}(\mathcal{L})}\right), \text { for all } \alpha_{l-1} \leq i \leq \alpha_{l}, 0 \leq l \leq r \text { and } 1 \leq j \leq \alpha_{r} .
$$

Since

$c_{i}\left(\frac{\pi_{2}^{*}\left(\left(\pi_{2}\right)_{*}(\mathcal{L})\right)}{J_{a-1}(\mathcal{L})}\right)=\frac{\theta^{i}}{i !}+\frac{\theta^{i-1}}{(i-1) !}(a \gamma+a(j+r) \eta+a(a-1)(j-1) \eta)-\frac{\theta^{i-1}}{(i-2) !} a(a+1) \eta$,

clearly $\left[X_{j, \alpha}\right]$ is a linear combination of $\theta^{j}, \theta^{j-1} \eta$ and $\theta^{j-1} \gamma$ in $H^{2 j}\left(C \times \operatorname{Pic}^{r+j}(C)\right)$. The intersection number $\left[X_{j, \alpha}\right] \cdot \theta$ can be interpreted as the number of line bundles $L_{C} \in$ $\mathrm{Pic}^{r+j}(C)$ satisfying the condition $\alpha_{i}^{L_{C}}(y) \geq \alpha_{i}$ for $i=0 \ldots r$ at an unspecified point $y \in$ $C$, and which, moreover, are also ramified at a fixed point $q \in C$, that is, $a_{r}^{L_{C}}(q) \geq r+1$.

Using this interpretation, the quantity $\sum_{\alpha \in \mathcal{P}_{1}} N_{g-j, \alpha}\left(\left[X_{g-j, \alpha}\right] \cdot \theta\right)$ can be expressed as the intersection number $\widetilde{C}_{j} \cdot \overline{\mathfrak{L i n}}_{d}^{r}(1)$ over the moduli space $\overline{\mathcal{M}}_{g, 1}$. Here $\mathfrak{L i n}_{d}^{r}(1)$ is the divisor on $\mathcal{M}_{g, 1}$ consisting of pointed curves $[C, q]$ such that there exists $L \in W_{d}^{r}(C)$ with $h^{0}\left(C, L \otimes \mathcal{O}_{C}(-(r+1) q) \geq 1\right.$, while $\widetilde{C}_{j}=\left\{C \cup_{y} D, q\right\}_{y \in C} \subset \Delta_{j: 1} \subset \overline{\mathcal{M}}_{g, 1}$ is the test curve obtained by varying the point of attachment $y$ on the genus $j$ component, while the marked point $q \in C$ remains fixed. The class of $\overline{\mathfrak{L i n}}_{d}^{r}(1)$ is computed in the course of the proof of Theorem 4.6 and one has

$$
\overline{\mathfrak{L i n}}_{d}^{r}(1) \equiv \mu\left((g+3) \lambda-\frac{g+1}{6} \delta_{i r r}-\sum_{j=1}^{g-1} \delta_{j: 1}\right)+\nu\left(-\lambda+\psi-\sum_{j=1}^{g-1}\left(\begin{array}{c}
g-j+1 \\
2
\end{array}\right) \delta_{j: 1}\right),
$$

where

$$
\nu=\frac{r(r+2)}{(r s+s-1)(r s+s+1)} \text { and } \mu=\frac{r(r+1)(r+2)(s-1)(s+1)(r s+s+4)}{2(s+r+1)(r s+s-2)(r s+s-1)(r s+s+1)} .
$$

Since $\widetilde{C}_{j} \cdot \psi=1, \widetilde{C}_{j} \cdot \delta_{g-j: 1}=1$ (the only point of intersection corresponds to $y=q \in C$ ), $\widetilde{C}_{j} \cdot \delta_{j: 1}=-(2 j-1)$, while $\widetilde{C}_{j} \cdot \lambda=\widetilde{C}_{j} \cdot \delta_{i: 1}=0$ for $i \neq j, g-j$, we can compute that

$$
\begin{aligned}
& \frac{B_{j}}{c_{r}}=\frac{s-1}{(j-1) c_{r}} \sum_{\alpha \in \mathcal{P}_{1}} N_{g-j, \alpha}\left(\left[X_{j, \alpha}\right] \cdot \theta\right)=\frac{s-1}{(j-1) c_{r}} \widetilde{C}_{j} \cdot \overline{\mathfrak{L i n}}_{d}^{r}(1)= \\
= & \frac{4(s-1) j\left(2 j s^{3}+j s^{2}-2 j s-2 j+4 s^{3}+4 s^{2}-3 s\right)\left(2 s^{2}+s-j\right)}{\left(2 s^{2}+s-2\right)(3 s+1)(2 s-1)(j-1)} \geq
\end{aligned}
$$




$$
\geq \frac{B_{0}}{c_{r}}=\frac{s\left(8 s^{6}-8 s^{5}-2 s^{4}+s^{2}+11 s+2\right)}{3\left(2 s^{2}+s-2\right)(3 s+1)(2 s-1)} .
$$

This finishes the proof and shows that $s\left(\sigma_{*}\left(\mathcal{G}_{0,2}-\mathcal{H}_{0,2}\right)\right)=A / B_{0}$.

As we have already pointed out, Theorem 1.1 produces only virtual divisors on $\overline{\mathcal{M}}_{g}$ of slope $<6+12 /(g+1)$. To get actual divisors one has to show that the vector bundle map $\phi: \mathcal{H}_{i, 2} \rightarrow \mathcal{G}_{i, 2}$ is generically non-degenerate. We carry this out in the case $i=0$ and we produce for the first time an infinite sequence of genuine counterexamples to the Slope Conjecture.

Proof of Theorem 1.5. From Brill-Noether theory one knows that there exists a unique component of $\widetilde{\mathfrak{G}}_{d}^{r}$ which maps onto $\widetilde{\mathcal{M}}_{g}$. Moreover, if $(C, L) \in \mathfrak{G}_{d}^{r}$ is such that $L \in$ $W_{d}^{r}(C)-W_{d}^{r+1}(C)$ corresponds to an embedding $C \subset \mathbf{P}^{r}$, then a sufficient condition for the smoothness of $\mathfrak{G}_{d}^{r}$ at $[C, L]$ is that $H^{1}\left(N_{C / \mathbf{P}^{r}}\right)=0$, and then, the differential $(d \sigma)_{[C, L]}$ is surjective if and only if the Petri map $\mu_{0}(C): H^{0}(L) \otimes H^{0}\left(K_{C} \otimes L^{\vee}\right) \rightarrow H^{0}\left(K_{C}\right)$ is injective (see e.g. [AC2]). In our situation, it is then enough to produce a Brill-NoetherPetri general smooth curve $C \subset \mathbf{P}^{2 s}$ having degree $2 s(s+1)$ and genus $s(2 s+1)$ such that $C$ does not sit on any quadrics, that is $H^{0}\left(\mathcal{I}_{C / \mathbf{P}^{2 s}}(2)\right)=H^{1}\left(\mathcal{I}_{C / \mathbf{P}^{2 s}}(2)\right)=0$. We carry this out inductively: for each $0 \leq a \leq s$, we construct a smooth non-degenerate curve $C_{a} \subset \mathbf{P}^{s+a}$ with $\operatorname{deg}\left(C_{a}\right)=\left(\begin{array}{c}s+a+1 \\ 2\end{array}\right)+a$ and $g\left(C_{a}\right)=\left(\begin{array}{c}s+a+1 \\ 2\end{array}\right)+a-s$, such that $C_{a}$ satisfies the Petri Theorem (in particular $H^{1}\left(C_{a}, N_{C_{a} / \mathbf{P}^{s+a}}\right)=0$ ), and such that the multiplication map $\mu_{2}: \operatorname{Sym}^{2} H^{0}\left(C_{a}, \mathcal{O}_{C_{a}}(1)\right) \rightarrow H^{0}\left(C_{a}, \mathcal{O}_{C_{a}}(2)\right)$ is surjective (or equivalently, an isomorphism).

To construct $C_{0} \subset \mathbf{P}^{s}$ we consider the White surface $S=\mathrm{Bl}_{\left\{p_{1}, \ldots, p_{\delta}\right\}}\left(\mathbf{P}^{2}\right) \subset \mathbf{P}^{s}$ obtained by blowing-up $\mathbf{P}^{2}$ at general points $p_{1}, \ldots, p_{\delta} \in \mathbf{P}^{2}$ where $\delta=\left(\begin{array}{c}s+1 \\ 2\end{array}\right)$, and embedding it via the linear system $\left|s h-\sum_{i=1}^{\delta} E_{p_{i}}\right|$. Here $h$ is the class of a line on $\mathbf{P}^{2}$. It is known that $S \subset \mathbf{P}^{s}$ is a projectively Cohen-Macaulay surface and its ideal is generated by the $(3 \times 3)$-minors of a certain $(3 \times s)$-matrix of linear forms (see e.g. [GG] even though these surfaces have been studied in the classical literature by T.G. Room in $[\mathbb{R}])$. The Betti diagram of $S \subset \mathbf{P}^{s}$ is the same as that of the ideal of $(3 \times 3)$-minors of a $(3 \times s)$-matrix of indeterminates. In particular, we have that $H^{i}\left(\mathcal{I}_{S / \mathbf{P}^{s}}(2)\right)=0$ for $i=0,1$. On $S$ we consider a generic smooth curve $C \equiv(s+1) h-\sum_{i=1}^{\delta} E_{p_{i}}$. We find that the embedded curve $C \subset S \subset \mathbf{P}^{s}$ has $\operatorname{deg}(C)=\left(\begin{array}{c}s+1 \\ 2\end{array}\right)$ and $g(C)=\left(\begin{array}{l}s \\ 2\end{array}\right)$. From the exact sequence

$$
0 \longrightarrow \mathcal{I}_{S / \mathbf{P}^{s}}(1) \longrightarrow \mathcal{I}_{C / \mathbf{P}^{s}}(1) \longrightarrow \mathcal{I}_{C / S}(1) \longrightarrow 0,
$$

using also that $H^{1}\left(\mathcal{I}_{S / \mathbf{P}^{s}}(1)\right)=0$ and that $H^{1}\left(\mathcal{I}_{C / S}(1)\right)=0$ (e.g. by Riemann-Roch), we find that $H^{1}\left(\mathcal{I}_{C / \mathbf{P}^{s}}(1)\right)=0$ and $H^{1}\left(\mathcal{O}_{C}(1)\right)=0$, hence $h^{0}\left(\mathcal{O}_{C}(1)\right)=s+1$. Furthermore, since $H^{0}\left(\mathcal{I}_{S / \mathbf{P}^{s}}(2)\right)=H^{1}\left(\mathcal{I}_{S / \mathbf{P}^{s}}(2)\right)=0$, we obtain that $H^{1}\left(\mathcal{I}_{C / \mathbf{P}^{s}}(2)\right)=0$. Finally, since $H^{1}\left(\mathcal{O}_{C}(1)\right)=0$, it follows trivially that $H^{1}\left(N_{C / \mathbf{P}^{s}}\right)=0$ and $\mu_{0}(C)$ is injective, being a map with source the trivial vector space. Even though $[C] \in \mathcal{M}_{g(C)}$ itself is not a Petri general curve, the map $\mathcal{H}_{C} \rightarrow \mathcal{M}_{g(C)}$ from the Hilbert scheme $\mathcal{H}_{C}$ of curves $C^{\prime} \subset \mathbf{P}^{s}$ with $\operatorname{deg}\left(C^{\prime}\right)=\operatorname{deg}(C)$ and $g\left(C^{\prime}\right)=g(C)$, is smooth and dominant around the point $[C] \in \mathcal{H}_{C}$, hence a generic deformation $\left[C_{0} \hookrightarrow \mathbf{P}^{s}\right] \in \mathcal{H}_{C}$ of $\left[C \hookrightarrow \mathbf{P}^{s}\right]$ will be Petri general and still satisfy the condition $H^{1}\left(\mathcal{I}_{C_{0} / \mathbf{P}^{s}}(2)\right)=0$. 
Assume now that we have constructed a Petri general curve $C_{a} \subset \mathbf{P}^{s+a}$ with all the desired properties. We pick general points $p_{1}, \ldots, p_{s+a+2} \in C_{a}$ with the property that if $\Delta:=p_{1}+\cdots+p_{s+a+2} \in \operatorname{Sym}^{s+a+2} C_{a}$, then the variety

$$
T:=\left\{(M, V) \in W_{d\left(C_{a}\right)+s+a+2}^{s+a+1}\left(C_{a}\right): \operatorname{dim}\left(V \cap H^{0}\left(C_{a}, M \otimes \mathcal{O}_{C_{a}}(-\Delta)\right)\right) \geq s+a+1\right\}
$$

of linear series having an $(s+a+2)$-fold point along $\Delta$, has the expected dimension $\rho\left(g\left(C_{a}\right), s+a+1, d\left(C_{a}\right)+s+a+2\right)-(s+a+1)^{2}$. We identify the projective space $\mathbf{P}^{s+a}$ containing $C_{a}$ with a hyperplane $H \subset \mathbf{P}^{s+a+1}$ and choose a linearly normal elliptic curve $E \subset \mathbf{P}^{s+a+1}$ such that $E \cap H=\left\{p_{1}, \ldots, p_{s+a+2}\right\}$. The fact that such an $E$ exists is an easy consequence of the vanishing $H^{1}\left(N_{E / \mathbf{P}^{s+a+1}}(-1)\right)=0$ for each elliptic curve $E$ embedded by a complete linear series; the vanishing itself is a consequence of the fact that $N_{E / \mathbf{P}^{s+a+1}}$ is a poly-stable vector bundle (cf. [GL], Theorem 4.1), having the property that $\left.\mu\left(N_{E / \mathbf{P}^{s+a+1}}(-1)\right)>1\right)$. We now set $X:=C_{a} \cup_{\left\{p_{1}, \ldots, p_{s+a+2}\right\}} E \hookrightarrow \mathbf{P}^{s+a+1}$ and then $\operatorname{deg}(X)=p_{a}(X)+s$. From the exact sequence

$$
0 \longrightarrow \mathcal{O}_{E}\left(-p_{1}-\cdots-p_{s+a+2}\right) \longrightarrow \mathcal{O}_{X} \longrightarrow \mathcal{O}_{C_{a}} \longrightarrow 0,
$$

we can write that $h^{0}\left(\mathcal{O}_{X}(1)\right) \leq h^{0}\left(\mathcal{O}_{C_{a}}(1)\right)+h^{0}\left(\mathcal{O}_{E}\right)=s+a+2$, hence $h^{0}\left(\mathcal{O}_{X}(1)\right)=$ $s+a+2$ and $h^{1}\left(\mathcal{O}_{X}(1)\right)=a+1$. One can also write the exact sequence

$$
0 \longrightarrow \mathcal{I}_{E / \mathbf{P}^{s+a+1}}(1) \longrightarrow \mathcal{I}_{X / \mathbf{P}^{s+a+1}}(2) \longrightarrow \mathcal{I}_{C_{a} / H}(2) \longrightarrow 0
$$

from which we obtain that $H^{1}\left(\mathcal{I}_{X / \mathbf{p}^{s+a+1}}(2)\right)=0$, hence by a dimension count also $H^{0}\left(\mathcal{I}_{X / \mathbf{P}^{s+a+1}}(2)\right)=0$, that is, $X$ and every deformation of $X$ inside $\mathbf{P}^{s+a+1}$ will lie on no quadrics.

Next we show that $X \hookrightarrow \mathbf{P}^{s+a+1}$ can be deformed to an embedding of a smooth curve $C_{a+1}$ in $\mathbf{P}^{s+a+1}$ such that $H^{1}\left(N_{C_{a+1} / \mathbf{P}^{s+a+1}}\right)=0$. We choose an $(s+a+2)$ dimensional subspace $H^{0}\left(\mathcal{O}_{C_{a}}(1)\right) \subset V \subset H^{0}\left(\mathcal{O}_{C_{a}}(1) \otimes \mathcal{O}_{C_{a}}(\Delta)\right)$ giving a map $f$ : $C_{a} \rightarrow \mathbf{P}^{s+a+1}$ such that $f\left(p_{1}\right)=\cdots=f\left(p_{s+a+2}\right)=p$. If we denote by $\widetilde{\mathbf{P}}^{s+a+1}$ the blowup of $\mathbf{P}^{s+a+1}$ at $p$, by choosing $V$ suitably we may assume that $f$ lifts to an embedding $\tilde{f}: C_{a} \hookrightarrow \widetilde{\mathbf{P}}^{s+a+1}$ which projected from $p$ gives rise to the original embedding $C_{a} \hookrightarrow H$. We consider another copy of $\mathbf{P}^{s+a+1}$ which we denote by $\mathbf{P}_{1}^{s+a+1}$ and we denote by $Z$ the scheme obtained by gluing $\mathbf{P}_{1}^{s+a+1}$ and $\widetilde{\mathbf{P}}^{s+a+1}$ along $H$, where we identify the exceptional divisor of $\widetilde{\mathbf{P}}^{s+a+1}$ with $H \subset \mathbf{P}^{s+a+1}$ via the projection from $p$. There is a natural map $h: Z \rightarrow \mathbf{P}^{s+a+1}$ which on $\mathbf{P}_{1}^{s+a+1}$ is the identity while on $\widetilde{\mathbf{P}}^{s+a+1}$ is the projection from $p$. Via this map, the inclusion $X \hookrightarrow \mathbf{P}^{s+a+1}$ lifts to an embedding $X \hookrightarrow Z$. Note that $Z$ is a degeneration of $\mathbf{P}^{s+a+1}$ something which can be seen by blowing-up the codimension 2 subscheme $H \times\{0\}$ of $\mathbf{P}^{s+a+1} \times \mathbf{P}^{1}$. If we denote by $\mathcal{X}$ the total space of the blow-up and by $\epsilon: \mathcal{X} \rightarrow \mathbf{P}^{1}$ the projection onto the second component, then for $t \neq 0$ we have that $\epsilon^{-1}(t)=\mathbf{P}^{s+a+1}$, whereas $\epsilon^{-1}(0)=\mathbf{P} \cup E$, where $\mathbf{P}$ is the strict transform of $\mathbf{P}^{s+a+1} \times\{0\}$ which is isomorphic to $\mathbf{P}^{s+a+1}$, while $E=\mathbf{P}\left(\mathcal{O}_{H} \oplus \mathcal{O}_{H}(1)\right)$ is the exceptional divisor, which is isomorphic to $\mathbf{P}^{s+a+1}$ blown-up at a point. In the special fibre, $\mathbf{P}$ and $E$ are joined along a divisor which is $H$ inside $\mathbf{P}$.

We can write down the standard exact sequences of normal bundles

$$
0 \longrightarrow N_{E / \mathbf{P}^{s+a+1}} \otimes \mathcal{O}_{E}(-\Delta) \longrightarrow N_{X / Z} \longrightarrow N_{C_{a} / \widetilde{\mathbf{P}}^{s+a+1}} \longrightarrow 0 \text { and }
$$




$$
0 \longrightarrow \mathcal{O}_{C_{a}}(1) \otimes \mathcal{O}_{C_{a}}(2 \Delta) \longrightarrow N_{C_{a} / \widetilde{\mathbf{P}}^{s+a+1}} \longrightarrow N_{C_{a} / H} \longrightarrow 0
$$

from which it easily follows that $H^{1}\left(N_{X / Z}\right)=0$ (Use the hypothesis $H^{1}\left(N_{C_{a} / \mathbf{P}^{s+a}}\right)=0$ and that $H^{1}\left(N_{E / \mathbf{p}^{s+a+1}} \otimes \mathcal{O}_{E}(-\Delta)\right)=0$ because $N_{E / \mathbf{p}^{s+a+1}}$ is semi-stable). Thus the space of deformations of $X$ in $Z$ is unobstructed of dimension $h^{0}\left(N_{X / Z}\right)$. On the other hand by general theory, the space $T_{(X, Z)}^{1}$ of infinitesimal deformations of the pair $(X, Z)$ has dimension at least $\chi\left(N_{X / Z}\right)+1=h^{0}\left(N_{X / Z}\right)+1$, which shows that there exists a deformation of $(X, Z)$ in which $Z$ deforms non-trivially. But $\operatorname{dim}\left(T_{Z}^{1}\right)=1$, that is, the only possible deformation of $Z$ is the smoothing to $\mathbf{P}^{s+a+1}$ previously described, and in this deformation the map $X \hookrightarrow Z$ will deform to an embedding $C_{a+1} \hookrightarrow \mathbf{P}^{s+a+1}$ of a smooth curve, which proves our claim. We are left with showing that the dimension estimate

$$
\operatorname{dim}\left(W_{d\left(C_{a+1}\right)}^{s+a+1}\left(C_{a+1}\right)\right)=\rho\left(g\left(C_{a+1}\right), s+a+1, d\left(C_{a+1}\right)\right)
$$

holds. Assuming for a moment (8) has been proved, since the vanishing condition $H^{1}\left(N_{C_{a+1} / \mathbf{P}^{s+a+1}}\right)=0$ guarantees the local smoothness of the scheme $\mathfrak{G}_{d\left(C_{a+1}\right)}^{s+a+1}$, it follows that the morphism $\mathfrak{G}_{d\left(C_{a+1}\right)}^{s+a+1} \rightarrow \mathcal{M}_{g\left(C_{a+1}\right)}$ is dominant in a neighbourhood of the point $\left[C_{a+1} \hookrightarrow \mathbf{P}^{s+a+1}\right]$. Therefore the curve $C_{a+1} \subset \mathbf{P}^{s+a+1}$ can be chosen to be Petri general as well, which enables us to continue the induction.

We return to proving (8) and denote by $\mathcal{U}$ the versal deformation space of $[X] \in$ $\overline{\mathcal{M}}_{g\left(C_{a+1}\right)}$ and by $\phi: \mathcal{C} \rightarrow \mathcal{U}$ the universal family such that $\phi^{-1}(0)=X$, where $0 \in$ $\mathcal{U}$. Then in a way similar to [EH1], Theorem 3.3, one can construct a quasi-projective variety $\sigma: \tilde{\mathfrak{G}}_{d\left(C_{a+1}\right)}^{s+a+1} \rightarrow \mathcal{U}$ of limit linear series such that for points $u \in \mathcal{U}$ with $C_{u}=$ $\phi^{-1}(u)$ being smooth, we have that $\sigma^{-1}(u)=G_{d\left(C_{a+1}\right)}^{s+a+1}\left(C_{u}\right)$, whereas $\sigma^{-1}(0)$ consists of the following data: an underlying line bundle $\mathcal{L}$ on $X$ together with linear series $\left\{L_{a}, V_{a} \in G\left(s+a+2, H^{0}\left(X, L_{a}\right)\right)\right\}$ and $\left\{L_{E}, V_{E} \in G\left(s+a+2, H^{0}\left(X, L_{E}\right)\right)\right\}$ such that the following conditions are satisfied (see also [Est], Theorem 1 ):

(1) The line bundles $L_{a}$ and $L_{E}$ on $X$ are suitable twists of $\mathcal{L}$ by multiples of the divisor $\Delta$ : precisely there exists an integer $l$ such that $L_{a \mid C_{a}}=L_{E \mid C_{a}} \otimes \mathcal{O}_{C_{a}}(l \Delta)$ and $L_{a \mid E}=L_{E \mid E} \otimes \mathcal{O}_{E}(-l \Delta)$. Moreover $\operatorname{deg}\left(L_{a} \mid C_{a}\right)+\operatorname{deg}\left(L_{E \mid E}\right)=d\left(C_{a+1}\right)+$ $l(s+a+2)$.

(2) The restriction maps $V_{a} \rightarrow H^{0}\left(C_{a}, L_{a \mid C_{a}}\right)$ and $V_{E} \rightarrow H^{0}\left(E, L_{E \mid E}\right)$ are both injective.

(3) The restriction maps $V_{a} \rightarrow H^{0}\left(E, L_{a \mid E}\right)$ and $V_{E} \rightarrow H^{0}\left(C_{a}, L_{E \mid C_{a}}\right)$ are both nonzero.

(4) If $l$ is the integer defined above and $\left(a_{0} \leq \ldots \leq a_{s+a+1}\right)$ denotes the vanishing sequence of $\left(L_{a \mid C_{a}}, V_{a+1}\right)$ with respect to the divisor $\Delta \in$ Sym $^{s+a+2} C_{a}$ while $\left(b_{0} \leq \cdots \leq b_{s+a+1}\right)$ denotes the vanishing sequence of $\left(L_{E \mid E}, V_{E}\right)$ with respect to $\Delta \in$ Sym $^{s+a+2} E$, then we have the inequalities $a_{i}+b_{s+a+1-i} \geq l$ for all indices $0 \leq i \leq s+a+1$ (see also [Est], Proposition 6).

We also have the dimension estimate $\operatorname{dim}(\mathcal{G}) \geq \operatorname{dim}(\mathcal{U})+\rho\left(g\left(C_{a+1}\right), s+a+1, d\left(C_{a+1}\right)\right)$, (by construction, see also [EH1]), thus, in order to prove (8) it suffices to show that

$$
\operatorname{dim}\left(\sigma^{-1}(0)\right)=\rho\left(g\left(C_{a+1}\right), s+a+1, d\left(C_{a+1}\right)\right)=\rho\left(g\left(C_{a}\right), s+a, d\left(C_{a}\right)\right)-a
$$


(here by dimension we mean the smallest dimension of an irreducible component). It is now easy to describe the fibre $\sigma^{-1}(0)$ in a neighbourhood of the point corresponding to a smoothing of the embedding $X \hookrightarrow \mathbf{P}^{s+a+1}$ : if $\left\{L_{a}, V_{a}\right\},\left\{L_{E}, V_{E}\right\}$ is a limit linear series on $X$, then the aspect corresponding to $E$ is just a very ample line on $X$ giving the embedding into $\mathbf{P}^{s+a+1}$, that is, $L_{E \mid C_{a}} \in W_{d\left(C_{a}\right)}^{s+a}\left(C_{a}\right)$ and $L_{E \mid E}=\mathcal{O}_{E}(\Delta)$, whereas the aspect corresponding to $C_{a}$ is described by $L_{a \mid E}=\mathcal{O}_{E}$ and $L_{a \mid C_{a}}=\mathcal{O}_{C_{a}}(\Delta) \otimes L_{E \mid C_{a}}$ (and in particular $l=1$ ). The only possibility for the vanishing sequences of the $E$ and $C_{a}$ aspects is that $\left(a_{0}, \ldots, a_{s+a+1}\right)=(0,1, \ldots, 1)$ and $\left(b_{0}, \ldots, b_{s+a+1}\right)=(0, \ldots, 0,1)$. This shows that locally, $\sigma^{-1}(0)$ is isomorphic to the variety of line bundles $\mathcal{L} \in \operatorname{Pic}^{d\left(C_{a+1}\right)}(X)$ such that $\mathcal{L}_{\mid E}=\mathcal{O}_{E}, h^{0}(X, \mathcal{L}) \geq s+a+2$ and $h^{0}\left(C_{a}, \mathcal{L}_{\mid C_{a}}(-\Delta)\right) \geq s+a+1$ (Loosely speaking this is the subscheme consisting of those $L_{a} \in W_{d\left(C_{a}\right)}^{s+a}\left(C_{a}\right)$ for which there exists a section $\tau \in \mathbf{P}\left(H^{0}\left(L_{a} \otimes \mathcal{O}(\Delta)\right) / H^{0}\left(L_{a}\right)\right)$ which glues to the unique section of the trivial bundle $\mathcal{O}_{E}$ at the points of attachment $\left.p_{1}, \ldots, p_{s+a+2}\right)$. Thus locally $\sigma^{-1}(0)$ is a $\left(\mathbb{C}^{*}\right)^{s+a+1}$-bundle over the subvariety $T$ of $W_{d\left(C_{a+1}\right)}^{s+a+1}$ having an $(s+a+2)$-fold point along the divisor $\Delta$, and by our inductive hypothesis we know that $\operatorname{dim}(T)=$ $\operatorname{dim}\left(W_{d\left(C_{a+1}\right)}^{s+a+1}\left(C_{a}\right)\right)-(s+a+1)^{2}$. It follows that

$$
\operatorname{dim}\left(\sigma^{-1}(0)\right)=\operatorname{dim}(T)+s+a+1=\rho\left(g\left(C_{a+1}\right), s+a+1, d\left(C_{a+1}\right)\right),
$$

and this finishes the proof.

Remark 2.13. It is natural to wonder whether (8) could not be proved more easily by showing directly that the Petri map $\mu_{0}(X): H^{0}\left(\mathcal{O}_{X}(1)\right) \otimes H^{0}\left(\omega_{X}(-1)\right) \rightarrow H^{0}\left(\omega_{X}\right)$ is injective. Indeed Theorem 1.3 from [CR] seems to imply this to be the case based on the inductive hypothesis that $\mu_{0}\left(C_{a}\right)$ is injective whereas $\mu_{0}(E)$ is injective for trivial reasons. That claim is incorrect: from the exact sequence $0 \longrightarrow \omega_{E} \longrightarrow \omega_{X} \longrightarrow \omega_{C_{a}}(\Delta) \longrightarrow 0$, we find the isomorphism $H^{0}\left(\omega_{X}(-1)\right)=H^{0}\left(\omega_{C_{a}}(-1) \otimes \mathcal{O}_{C_{a}}(\Delta)\right)$ and then a simple analysis shows that $H^{0}\left(\mathcal{O}_{E}\right) \otimes H^{0}\left(\omega_{C_{a}}(-1)\right) \subset H^{0}\left(\mathcal{O}_{X}(1)\right) \otimes H^{0}\left(\omega_{X}(-1)\right)$ is an $a$-dimensional subspace lying entirely inside $\operatorname{Ker}\left(\mu_{0}(X)\right)$.

\section{THE ClASS OF THE GIESEKER-PETRI DIVISORS}

In this section we prove Theorem 1.6. We use the same strategy as in the previous section and we intersect $\overline{\mathcal{G P}}_{g, d}^{r}$ with the test curves $C^{0}, C^{1}$ and $C^{j}$ for $[g / 2] \leq j \leq g-2$. Recall that we have constructed a rank $r+1$ vector bundle $\mathcal{G}_{0,1}$ over the variety $\widetilde{\mathfrak{G}}_{d}^{r}$ (cf. Proposition 2.10). As usual, we denote by $\mathbb{E}$ the Hodge bundle over $\overline{\mathcal{M}}_{g}$.

Proposition 3.1. There exists a vector rank $s$ vector bundle $\mathcal{N}$ over $\widetilde{\mathfrak{G}}_{d}^{r}$ together with a morphism $\mathcal{G}_{0,1} \otimes \mathcal{N} \rightarrow \sigma^{*}(\mathbb{E})$ of vector bundles of the same rank over $\widetilde{\mathfrak{G}}_{d}^{r}$ such that the fibres of $\mathcal{N}$ admit the following description:

- If $(C, L) \in \mathfrak{G}_{d}^{r}$, then $\mathcal{N}(C, L)=H^{0}\left(C, K_{C} \otimes L^{\vee}\right)$.

- If $t=\left(C \cup_{y} E, L_{C}, l_{E}\right) \in \sigma^{-1}\left(\Delta_{1}^{0}\right)$, where $L \in W_{d}^{r}(C)$ is such that $h^{0}(L(-2 y))=r$, then $\mathcal{N}(t)=H^{0}\left(C, K_{C} \otimes L_{C}^{\vee} \otimes \mathcal{O}_{C}(2 y)\right)$.

- If $t=(C / y \sim q, L) \in \sigma^{-1}\left(\Delta_{0}^{0}\right)$, where $y, q \in C$ and $L \in W_{d}^{r}(C)$ is such that $h^{0}(L(-y-q))=h^{0}(L)-1$, then $\mathcal{N}(t)=H^{0}\left(C, K_{C} \otimes L^{\vee} \otimes \mathcal{O}_{C}(y+q)\right)$. 
- If $t=\left(C \cup_{y} D, l_{C}, l_{D}\right) \in \sigma^{-1}\left(\Delta_{j}^{0}\right)$ where $[g / 2] \leq j \leq g-2, g(C)=j, g(D)=g-j$, then $\mathcal{N}(t)=H^{0}\left(C \cup_{y} D, \omega_{C \cup D} \otimes L^{\vee}\right)$, where $L=\left(l_{C}(-(r s-j) y), l_{D}(-(j+r) y)\right) \in$ $\mathrm{Pic}^{j+r}(C) \times \mathrm{Pic}^{r s-j}(D)$.

Note that over $\mathfrak{G}_{d}^{r}$ the morphism $\mathcal{G}_{0,1} \otimes \mathcal{N} \rightarrow \sigma^{*}(\mathbb{E})$ is simply the Petri multiplication map. We start the proof of Theorem 1.6 by expanding $\left[\overline{\mathcal{G P}}_{g, d}^{r}\right]$ in $\operatorname{Pic}\left(\overline{\mathcal{M}}_{g}\right)$ :

$$
\overline{\mathcal{G P}}_{g, d}^{r} \equiv a \lambda-b_{0} \delta_{0}-\cdots-b_{[g / 2]} \delta_{[g / 2]} .
$$

We show that the coefficients $a, b_{0}$ and $b_{1}$ as well as $s\left(\overline{\mathcal{G P}}_{g, d}^{r}\right)$ can be read off from the bundle map $\mathcal{G}_{0,1} \otimes \mathcal{N} \rightarrow \sigma^{*}(\mathbb{E})$.

Proposition 3.2. One has the relation $a-12 b_{0}+b_{1}=0$. Moreover, one has the identity $\overline{\mathcal{G P}}_{g, d}^{r} \equiv \sigma_{*}\left(c_{1}\left(\sigma^{*}(\mathbb{E})\right)-c_{1}\left(\mathcal{G}_{0,1} \otimes \mathcal{N}\right)\right)+\sum_{j=2}^{[g / 2]} d_{j} \delta_{j}$, where $d_{j} \geq 0$.

Proof. It is enough to show that if $[C, y] \in \mathcal{M}_{g-1,1}$ is a general pointed curve, then for every $L \in W_{d}^{r}(C)$ satisfying $h^{0}(L(-2 y))=r$ the multiplication map

$$
\mu_{0}(L, y): H^{0}(L) \otimes H^{0}\left(K_{C} \otimes L^{\vee} \otimes \mathcal{O}_{C}(2 y)\right) \rightarrow H^{0}\left(K_{C} \otimes \mathcal{O}_{C}(2 y)\right)
$$

is an isomorphism. This shows that the morphism $\mathcal{G}_{0,1} \otimes \mathcal{N} \rightarrow \sigma^{*}(\mathbb{E})$ is non-degenerate along each component of the divisor $\sigma^{-1}\left(\Delta_{1}^{0}\right)$ and the conclusion follows. To show that $\mu_{0}(L, y)$ is an isomorphism, we use a variation of the degeneration considered by Eisenbud and Harris to prove the Gieseker-Petri Theorem (cf. [EH4]). Precisely, we consider a 1-dimensional family of generically smooth pointed curves of genus $g-1$ $\pi: \mathcal{C} \rightarrow B$ with a section $\tau: B \rightarrow \mathcal{C}$, degenerating to a curve of compact type $C_{0}$ consisting of a string of rational components and $g$ elliptic components $E_{1}, \ldots, E_{g}$ such that the stable model of $C_{0}$ is $E_{1} \cup_{p_{1}} E_{2} \cup_{p_{2}} E_{3} \cup \cdots \cup_{p_{g-1}} E_{g-1}$. We assume moreover that the marked point specializes to a point $p_{0} \in E_{1}$ and we choose our degeneration general enough such that $p_{i}-p_{i-1} \in \operatorname{Pic}^{0}\left(E_{i}\right)$ is not a torsion point for all $1 \leq i \leq g-1$. By contradiction, we assume that for a general $t \in B$ there exists $L_{t} \in W_{d}^{r}\left(\pi^{-1}(t)\right)$ with $h^{0}\left(\pi^{-1}(t), L_{t} \otimes \mathcal{O}(-2 \tau(t))\right)=r$, such that $\mu_{0}\left(L_{t}, \tau(t)\right)$ has $\neq 0$ kernel. For $1 \leq i \leq g$ we denote by $L^{i} \in \operatorname{Pic}^{d}\left(C_{0}\right)$ the limit line bundle of the $L_{t}$ 's having the property that $\operatorname{deg}\left(L_{\mid E_{j}}^{i}\right)=0$ for $i \neq j$, hence $\operatorname{deg}\left(L_{\mid E_{i}}^{i}\right)=d$. Similarly, we define $M^{i} \in \operatorname{Pic}^{2 g-2-d}\left(C_{0}\right)$ to be the limit when $t \rightarrow 0$ of $K_{\pi^{-1}(t)} \otimes L_{t}^{\vee} \otimes \mathcal{O}_{\pi^{-1}(t)}(2 \tau(t))$ uniquely characterized by the property $\operatorname{deg}\left(M_{\mid E_{j}}^{i}\right)=0$ for $i \neq j$ and $\operatorname{deg}\left(M_{\mid E_{i}}^{i}\right)=2 g-2-d$. We denote by $\left\{\left(L_{\mid E_{i}}^{i}, V_{i} \in G\left(r+1, H^{0}\left(E_{i}, L_{\mid E_{i}}\right)\right)\right)\right\}$ and by $\left\{\left(M_{\mid E_{i}}^{i}, W_{i} \in G\left(r+1, H^{0}\left(E_{i}, M_{\mid E_{i}}\right)\right)\right)\right\}$ the limit linear series on $C_{0}$ corresponding to $L_{t}$ and $K_{\pi^{-1}(t)} \otimes L_{t}^{\vee}$ respectively as $t \rightarrow 0$. Reasoning along the lines of [EH4] or [F2], Proposition 5.2, for each $1 \leq i \leq g$ we find $\neq 0$ elements

$$
\rho_{i} \in \operatorname{Ker}\left\{V_{i} \otimes W_{i} \rightarrow H^{0}\left(E_{i}, L^{i} \otimes M_{\mid E_{i}}^{i}\right)\right\}
$$

satisfying $\operatorname{ord}_{p_{i}}\left(\rho_{i+1}\right) \geq \operatorname{ord}_{p_{i-1}}\left(\rho_{i}\right)+2$ for $1 \leq i \leq g-1$. Since both $V_{1}$ and $W_{1}$ have a cusp at $p_{0} \in E_{1}$, it follows that $\operatorname{ord}_{p_{1}}\left(\rho_{1}\right) \geq 2$, hence $\operatorname{ord}_{p_{g-1}}\left(\rho_{g}\right) \geq 2 g-2=\operatorname{deg}\left(L_{\mid E_{g}}^{g}\right)+$ $\operatorname{deg}\left(M_{\mid E_{g}}^{g}\right)$, which is a contradiction because $\rho_{g-1} \in H^{0}\left(E_{g}, L_{\mid E_{g}}^{g}\right) \otimes H^{0}\left(E_{g}, M_{\mid E_{g}}^{g}\right)$ being an element in the kernel of the multiplication map must be a tensor of rank $\geq 4$. 
Proposition 3.3. If $c_{r}$ is the constant defined in Lemma 2.6. then the $\delta_{1}$ coefficient in the expression of $\left[\overline{\mathcal{G P}}_{g, d}^{r}\right]$ is given by:

$$
b_{1}=c_{r} \frac{r(s-1)}{(s+r+1)(r s+s-2)}\left(3 r s^{2}+2 s^{2}+r^{2} s^{2}+7 s+6 r s+r^{2} s+2 r+2\right) .
$$

Proof. We fix a general curve $C$ of genus $g-1$ and consider the associated test curve $C^{1} \subset \Delta_{1}$. We view the curve $X \subset C \times W_{d}^{r}(C)$ defined in Proposition 2.11, as sitting inside $\widetilde{\mathfrak{G}}_{d}^{r}$. Then the projection $\pi_{1}: X \rightarrow C$ is the restriction of $\sigma: \widetilde{\mathfrak{G}}_{d}^{r} \rightarrow \widetilde{\mathcal{M}}_{g}$ once we identify $C$ with $C_{1}$ (Note that the degree of $\pi_{1}$ is precisely $c_{r}$ ). One can write the relation $(2 g-4) B_{1}=C^{1} \cdot \overline{\mathcal{G P}}_{g, d}^{r}=c_{1}\left(\sigma^{*}(\mathbb{E})_{\mid X}\right)-c_{1}\left(\mathcal{G}_{0,1 \mid X} \otimes \mathcal{N}_{\mid X}\right)$ and we are going to compute each term in this expression.

The restriction $\mathbb{E}_{\mid C^{1}}$ is identified with the vector bundle $\left(p_{2}\right)_{*}\left(p_{1}^{*}\left(K_{C}\right) \otimes \mathcal{O}(2 \Delta)\right)$, where $p_{1}, p_{2}: C \times C \rightarrow C$ are the two projections and $\Delta \subset C \times C$ is the diagonal. Using Grothendieck-Riemann-Roch for the map $p_{2}$, we find that

$$
c_{1}\left(\mathbb{E}_{\mid C^{1}}\right)=c_{1}\left(\left(p_{2}\right) !\left(p_{1}^{*}\left(K_{C}\right) \otimes \mathcal{O}(2 \Delta)\right)\right)=-2 g+4,
$$

hence $c_{1}\left(\sigma^{*}(\mathbb{E})\right)_{\mid X}=-(2 g-4) c_{r}$ (remember that $\left.c_{i}=c_{1}\left(\mathcal{E}^{\vee}\right)\right)$.

The fibre $\mathcal{N}_{\mid X}(y, L)$ is identified with $H^{0}\left(K_{C} \otimes L^{\vee}(2 y)\right)=H^{1}(L \otimes \mathcal{O}(-2 y))^{\vee}$. Keeping in mind that we have introduced the vector bundle map $\zeta$ in Proposition 2.11, we have an exact sequence over $X$

$$
0 \longrightarrow \operatorname{Ker}(\zeta)^{\vee} \longrightarrow \mathcal{N}_{\mid X}^{\vee} \longrightarrow \pi_{2}^{*}\left(R^{1} \pi_{2 *}\left(\mathcal{L}_{\mid C \times W_{d}^{r}(C)}\right)\right) \longrightarrow 0
$$

globalizing the cohomology exact sequence for each $(y, L) \in C \times W_{d}^{r}(C)$

$$
\cdots \longrightarrow H^{0}(L) \stackrel{\zeta^{\vee}}{\longrightarrow} H^{0}\left(L_{\mid 2 y}\right) \longrightarrow H^{1}(L(-2 y)) \longrightarrow H^{1}(L) \longrightarrow 0 .
$$

Hence $c_{1}\left(\mathcal{N}_{\mid X}^{\vee}\right)=\theta-c_{1}\left(\mathcal{E}^{\vee}\right)+c_{1}\left(\operatorname{Ker}(\zeta)^{\vee}\right)$. Using Proposition 2.11 we can write that

$$
\begin{aligned}
C^{1} \cdot \overline{\mathcal{G P}}_{g, d}^{r}=-(2 g-4) c_{r} \eta-(r+1-s) c_{1} \cdot[X]+(r+1) c_{1}\left(\operatorname{Ker}(\zeta)^{\vee}\right)= \\
\quad-(2 g-4) c_{r} \eta-(r+1-s)\left((2 d+2 g-4) c_{1} c_{r-1} \eta-6 c_{1} c_{r-2} \theta \eta\right)+ \\
+(r+1)\left((2 d+2 g-4) c_{r-1} \theta \eta-6 c_{r-2} \theta^{2} \eta\right)+(r+1) c_{1}\left(\operatorname{Ker}(\zeta)^{\vee}\right) .
\end{aligned}
$$

To compute $c_{1}\left(\operatorname{Ker}(\zeta)^{\vee}\right)$ we use once more $[\mathrm{HT}]$ and we find the following relation in $H^{t o p}\left(C \times W_{d}^{r}(C)\right)$ :

$$
c_{1}\left(\operatorname{Ker}(\zeta)^{\vee}\right)=c_{r+1}\left(\frac{\pi_{2}^{*}\left(\mathcal{E}^{\vee}\right)}{J_{1}\left(\mathcal{L}^{\vee}\right)}\right)=(2 d+2 g-4) c_{r} \eta-6 \eta \theta c_{r-1} .
$$

Combining the last two relations and then applying Lemma 2.6 we obtain the formula for $b_{1}$.

Proposition 3.4. The $\delta_{0}$ coefficient in the expression of $\left[\overline{\mathcal{G P}}_{g, d}^{r}\right]$ is given by:

$$
b_{0}=c_{r} \frac{r(r+1)(r+2)(s-1) s(s+1)(r s+s+4)}{6(r+s+1)(r s+s-2)(r s+s-1)} .
$$


Proof. We pick a general curve $C$ of genus $g-1$ and consider the test curve $C^{0} \subset \Delta_{\text {) }}$. Similarly to the proof of Proposition 3.3 we view the projection $\pi_{1}: Y \rightarrow C$ as the restriction of $\sigma: \widetilde{\mathfrak{G}}_{d}^{r} \rightarrow \widetilde{\mathcal{M}}_{g}$ over $C^{0}$. Then one has the relation $(2 g-2) b_{0}-b_{1}=$ $C^{0} \cdot \overline{\mathcal{G P}}_{g, d}^{r}=c_{1}\left(\sigma^{*}(\mathbb{E})_{\mid Y}\right)-c_{1}\left(\mathcal{G}_{0,1 \mid Y} \otimes \mathcal{N}_{\mid Y}\right)$. The Hodge bundle $\mathbb{E}_{\mid C^{0}}$ is identified with $\left(p_{2}\right)_{*}\left(p_{1}^{*}\left(K_{C}\right) \otimes \mathcal{O}\left(\Delta+\Gamma_{q}\right)\right)$, where $\Gamma_{q}=\{q\} \times C$, and it is easy to compute that $c_{1}\left(\sigma^{*}(\mathbb{E})_{\mid Y}\right)=c_{r}$. If we denote by $v$ the vector bundle morphism over $Y$ which globalizes the maps $H^{0}\left(L_{\mid y+q}\right)^{\vee} \rightarrow H^{0}(L)^{\vee}$ for each $(y, L) \in Y$, we obtain an exact sequence of vector bundles over $Y$

$$
0 \longrightarrow \operatorname{Ker}(v)^{\vee} \longrightarrow \mathcal{N}_{\mid Y}^{\vee} \longrightarrow \pi_{2}^{*}\left(R^{1} \pi_{2 *}\left(\mathcal{L}_{\mid C \times W_{d}^{r}(C)}\right)\right) \longrightarrow 0,
$$

from which we can compute $c_{1}\left(\mathcal{N}_{\mid Y}^{\vee}\right)$ if we use [HT] which in this case reads

$$
c_{1}\left(\operatorname{Ker}\left(v^{\vee}\right)\right)=c_{r+1}\left(\frac{\pi_{2}^{*}\left(\mathcal{E}^{\vee}\right)}{\mathcal{F}}\right),
$$

where $\mathcal{F}$ is the vector bundle on $C \times W_{d}^{r}(C)$ with fibre $\mathcal{F}(y, L)=H^{0}\left(L_{\mid y+q}\right)^{\vee}$. Finally, we write

$$
C^{0} \cdot \overline{\mathcal{G P}}_{g, d}^{r}=c_{r} \eta+\left((r+1)\left(\theta-c_{1}+c_{1}\right)\right) \cdot[Y]+(r+1)\left(c_{r}(d-1)-2 c_{r-1} \theta\right) \eta,
$$

which eventually leads to the stated formula.

To finish the proof of Theorem 1.6 it suffices to show that for $[g / 2] \leq j \leq g-2$, the coefficient of $\delta_{j}$ in the expression of $\sigma_{*}\left(c_{1}\left(\sigma^{*}(\mathbb{E})\right)-c_{1}\left(\mathcal{G}_{0,1} \otimes \mathcal{N}\right)\right)$ always exceeds the coefficient of $\delta_{0}$, which equals $b_{0}$ and was computed in Proposition 3.4. This is a calculation along the lines of the proof of Theorem 1.4 and we skip the details.

\section{FIVE WAYS OF CONSTRUCTING KOSZUL DIVISORS FOR POINTED CURVES}

In this section we construct Koszul divisors on moduli spaces of pointed curves. As an application we improve Logan's results on which $\overline{\mathcal{M}}_{g, n}$ 's are of general type.

We start by recalling a few things about divisor classes on $\overline{\mathcal{M}}_{g, n}$. For $0 \leq i \leq g$ and a set of indices $S \subset\{1, \ldots, n\}$, the boundary divisor $\Delta_{i: S}$ corresponds to the closure of the locus of nodal curves $C_{1} \cup C_{2}$, with $C_{1}$ smooth of genus $i, C_{2}$ smooth of genus $g-i$, and such that the marked points sitting on $C_{1}$ are precisely those labeled by $S$. We also introduce the divisor $\Delta_{i r r}$ consisting of irreducible pointed curves with one node. We denote by $\delta_{i: S} \in \operatorname{Pic}\left(\overline{\mathcal{M}}_{g, n}\right)$ the class of $\Delta_{i: S}$ and by $\delta_{i r r}$ that of $\Delta_{i r r}$. For each $1 \leq i \leq n$ we define the tautological class $\psi_{i}:=c_{1}\left(\mathbb{L}_{i}\right)$, where $\mathbb{L}_{i}$ is the line bundle on $\overline{\mathcal{M}}_{g, n}$ with fibre $\mathbb{L}_{i}\left(\left[C, x_{1}, \ldots, x_{n}\right]\right)=T_{x_{i}}^{\vee}(C)$ over each point $\left[C, x_{1}, \ldots, x_{n}\right] \in \overline{\mathcal{M}}_{g, n}$. It is well known that when $g \geq 3$, the Hodge class $\lambda$, the boundaries $\delta_{i r r}$ and $\delta_{i: S}$, and the tautological classes $\psi_{i}$ for $1 \leq i \leq n$, freely generate $\operatorname{Pic}\left(\overline{\mathcal{M}}_{g, n}\right)$.

\subsection{Divisors defined in terms of the Minimal Resolution Conjecture.}

We fix integers $g, r \geq 1$ and $0 \leq i \leq g$ and set $n:=(2 r+1)(g-1)-2 i$. We define a divisor on $\mathcal{M}_{g, n}$ consisting of smooth pointed curves $\left(C, x_{1}, \ldots, x_{n}\right)$ such that the points 
$x_{1}, \ldots, x_{n}$ fail the Minimal Resolution Conjecture for the canonical curve $C \stackrel{\left|K_{C}\right|}{\hookrightarrow} \mathbf{P}^{g-1}$ (see [FMP] for background on MRC). Precisely we define the locus

$\mathfrak{M r r c}_{g, i}^{r}:=\left\{\left[C, x_{1}, \ldots, x_{n}\right] \in \mathcal{M}_{g, n}: h^{1}\left(C, \wedge^{i} M_{K_{C}} \otimes K_{C}^{\otimes(r+1)} \otimes \mathcal{O}_{C}\left(-x_{1}-\cdots-x_{n}\right)\right) \geq 1\right\}$.

If we denote by $\Gamma:=x_{1}+\cdots+x_{n} \in C_{n}$, by Serre duality, the condition appearing in the definition of $\mathfrak{M r c}_{g, i}^{r}$ is equivalent to

$$
h^{0}\left(C, \wedge^{i} M_{K_{C}}^{\vee} \otimes \mathcal{O}_{C}(\Gamma) \otimes K_{C}^{\otimes(-r)}\right) \geq 1 \Longleftrightarrow \mathcal{O}_{C}(\Gamma) \otimes K_{C}^{\otimes(-r)} \in \Theta_{\wedge^{i} M_{K_{C}}^{\vee}},
$$

where we recall that for a stable vector bundle $E$ on $C$ having slope $\nu(E)=\nu \in \mathbb{Z}$, its theta divisor is the determinantal locus

$$
\Theta_{E}:=\left\{\eta \in \operatorname{Pic}^{g-\mu-1}(C): h^{0}(C, E \otimes \eta) \geq 1\right\} .
$$

The main result from [FMP] gives an identification $\Theta_{\wedge^{i} M_{K_{C}}^{\vee}}=C_{g-i-1}-C_{i}$, where the right hand side is one of the difference varieties associated to $C$. Thus one has an alternative description of points in $\mathfrak{M r c}_{g, i}^{r}$ : a point $\left(C, x_{1}, \ldots, x_{n}\right) \in \mathfrak{M r c}_{g, i}^{r}$ if and only if there exists $\exists D \in C_{i}$ such that $h^{0}\left(C, \mathcal{O}_{C}(\Gamma+D) \otimes K_{C}^{\otimes(-r)}\right) \geq 1$.

First we equip $\mathfrak{M r c}_{g, i}^{r}$ with a determinantal scheme structure. We consider the following cartesian diagram of stacks

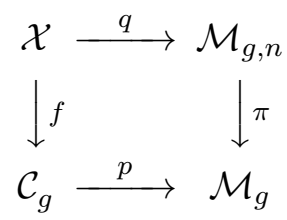

in which all the morphisms are smooth and $p$ (hence also $q$ ) is proper. We denote by $\omega_{p} \in \operatorname{Pic}\left(\mathcal{C}_{g}\right)$ the relative dualizing sheaf of the universal curve $p: \mathcal{C}_{g} \rightarrow \mathcal{M}_{g}$ and by $\mathbb{E}:=p_{*}\left(\omega_{p}\right)$ the Hodge bundle. We define the vector bundle $\mathcal{M}$ over $\mathcal{C}_{g}$ having rank $g-1$ as being the kernel of the evaluation map $p^{*} \mathbb{E} \longrightarrow \omega_{p}$. Thus for every $[C] \in \mathcal{M}_{g}$, we have $\left.\mathcal{M}\right|_{p^{-1}([C])} \simeq M_{K_{C}}$. For each $1 \leq j \leq n$ we have a section $q_{j}: \mathcal{M}_{g, n} \longrightarrow \mathcal{X}$ of $q$ given by $q_{j}\left(\left[C, x_{1}, \ldots, x_{n}\right]\right)=\left(\left[C, x_{1}, \ldots, x_{n}\right], x_{j}\right) \in \mathcal{X}$ and we set $E_{j}:=\operatorname{Im}\left(q_{j}\right)$, hence $E_{j}$ is a relative divisor over $\mathcal{M}_{g, n}$.

For integers $0 \leq a \leq i, b \geq r+2$ and $(a, b)=(0, r+1)$ we define the vector bundle

$$
\mathcal{A}_{a, b}:=q_{*}\left(f^{*}\left(\wedge^{a} \mathcal{M} \otimes \omega_{p}^{\otimes b}\right) \otimes \mathcal{O}_{\mathcal{X}}\left(-\sum_{j=1}^{n} E_{j}\right)\right),
$$

hence $\mathcal{A}_{a, b}\left(\left[C, x_{1}, \ldots, x_{n}\right]\right)=H^{0}\left(\wedge^{a} M_{K_{C}} \otimes K_{C}^{\otimes b} \otimes \mathcal{O}_{C}(-\Gamma)\right)$. To prove that $\mathcal{A}_{a, b}$ is locally free over $\mathcal{M}_{g, n}$, we use the fact that $M_{K_{C}}$ is a semi-stable vector bundle over $C$ and that $\mu\left(\wedge^{a} M_{K_{C}} \otimes K_{C}^{\otimes b}(-\Gamma)\right)>2 g-1$, hence

$$
R^{1} q_{*}\left(f^{*}\left(\wedge^{a} \mathcal{M} \otimes \omega_{p}^{\otimes b}\right) \otimes \mathcal{O}_{\mathcal{X}}\left(-\sum_{j=1}^{n} E_{j}\right)\right)=0
$$

whenever $b \geq r+2$. To reach the same conclusion in the case of the sheaf $\mathcal{A}_{0, r+1}$, we use that $H^{1}\left(K_{C}^{\otimes(r+1)}(-\Gamma)\right)^{\vee}=H^{0}\left(\mathcal{O}_{C}(\Gamma) \otimes K_{C}^{\otimes(-r)}\right)=0$, if $\Gamma \in C_{n}$ lies outside a subset 
of codimension $\geq 3$. Furthermore there is a vector bundle map

$$
\phi: \pi^{*}\left(\wedge^{i} \mathbb{E}\right) \otimes \mathcal{A}_{0, r+1} \rightarrow \mathcal{A}_{i-1, r+2}
$$

which over each point $\left[C, x_{1}, \ldots, x_{n}\right] \in \mathcal{M}_{g, n}$ corresponds to the natural map $\phi(C, \Gamma): \wedge^{i} H^{0}\left(K_{C}\right) \otimes H^{0}\left(K_{C}^{\otimes(r+1)} \otimes \mathcal{O}_{C}(-\Gamma)\right) \rightarrow H^{0}\left(\wedge^{i-1} M_{K_{C}} \otimes K_{C}^{\otimes(r+2)} \otimes \mathcal{O}_{C}(-\Gamma)\right)$. Note that $\operatorname{rank}\left(\mathcal{A}_{i-1, r+2}\right)=\operatorname{rank}\left(\pi^{*}\left(\wedge^{i} \mathbb{E}\right) \otimes \mathcal{A}_{0, r+1}\right)=2 i\left(\begin{array}{l}g \\ i\end{array}\right)$ and a simple argument using the exact sequence $0 \longrightarrow \wedge^{i} M_{K_{C}} \longrightarrow \wedge^{i} H^{0}\left(K_{C}\right) \otimes \mathcal{O}_{C} \longrightarrow \wedge^{i-1} M_{K_{C}} \otimes K_{C} \longrightarrow 0$ shows that $\mathfrak{M r c}_{g, i}^{r}$ is the degeneracy locus of the map $\phi$.

Proposition 4.1. The vector bundle morphism $\phi: \pi^{*}\left(\wedge^{i} \mathbb{E}\right) \otimes \mathcal{A}_{0, r+1} \rightarrow \mathcal{A}_{i-1, r+2}$ is generically non-degenerate. It follows that $\mathfrak{M r c}_{g, i}^{r}$ is a divisor on $\mathcal{M}_{g, n}$.

Proof. We show that $\phi$ is generically non-degenerate over the pull-back $\pi^{*}\left(\mathcal{H}_{g}\right)$ of the hyperelliptic locus. We fix a hyperelliptic curve $C$ of genus $g$ and we denote by $L \in$ $W_{2}^{1}(C)$ its hyperelliptic involution. By writing down the Euler sequence on $\mathbf{P}^{1}$ one shows that $M_{K_{C}}=\left(L^{\vee}\right)^{\oplus(g-1)}$, hence the condition $H^{1}\left(\wedge^{i} M_{K_{C}} \otimes K_{C}^{\otimes(r+1)} \otimes \mathcal{O}_{C}(-\Gamma)\right)=0$ is equivalent to $H^{0}\left(\mathcal{O}_{C}(\Gamma) \otimes L^{\otimes(i-r(g-1))}\right)=0$. This however is obvious because when $\Gamma \in C_{n}$ is a general divisor of degree $n$ then $\mathcal{O}_{C}(\Gamma) \otimes L^{\otimes(i-r(g-1))}$ is a general line bundle of degree $g-1$, therefore it has no global sections.

The main result here is the computation of the class of $\overline{\mathfrak{M r c}}_{g, i}^{r}$ :

Theorem 4.2. When $n=(2 r+1)(g-1)-2 i$, the locus $\mathfrak{M r c}_{g, i}^{r}$ is a divisor on $\mathcal{M}_{g, n}$ and the class of its compactification in $\overline{\mathcal{M}}_{g, n}$ is given by the following formula:

$$
\overline{\mathfrak{M r c}}_{g, i}^{r} \equiv \frac{1}{g-1}\left(\begin{array}{c}
g-1 \\
i
\end{array}\right)\left(a \lambda+c \sum_{j=1}^{n} \psi_{j}-b_{i r r} \delta_{i r r}-\sum_{j, s \geq 0,} b_{j: s} \sum_{|S|=s} \delta_{j: S}\right),
$$

where

$$
\begin{gathered}
c=r g+g-i-r-1, b_{i r r}=-\frac{1}{g-2}\left(\left(\begin{array}{c}
r+1 \\
2
\end{array}\right)(g-1)(g-2)+i(i+1+2 r-r g-g)\right), \\
a=-\frac{1}{g-2}\left((g-1)(g-2)\left(6 r^{2}+6 r+1\right)+i(24 r+10 i+10-10 g-12 r g)\right), \\
b_{0: s}=\left(\begin{array}{c}
s+1 \\
2
\end{array}\right)(g-1)+s(r g-r)-s i, \text { and } b_{j: s} \geq b_{0: s} \text { for } j \geq 1 .
\end{gathered}
$$

Remark 4.3. For $i=0, n=(2 r+1)(g-1)$, the divisor $\overline{\mathfrak{M r c}}_{g, 0}^{r}$ specializes to the locus of points $\left[C, x_{1}, \ldots, x_{(2 r+1)(g-1)}\right] \in \mathcal{M}_{g,(2 r+1)(g-1)}$ such that $\sum_{j=1}^{(2 r+1)(g-1)} x_{j} \in\left|K_{C}^{\otimes r}\right|$ and Theorem 4.2 gives that:

$\overline{\mathfrak{M r c}}_{g, 0}^{r} \equiv-\left(6 r^{2}+6 r+1\right) \lambda+(r+1)\left(\sum_{j=1}^{(2 r+1)(g-1)} \psi_{j}\right)+\left(\begin{array}{c}r+1 \\ 2\end{array}\right) \delta_{i r r}-(2 r+3) \sum_{|S|=2} \delta_{0: S}-\cdots$

By letting all the marked points coalesce and using the standard formulas for pushing forward products of tautological classes (cf. e.g. [FMP] or [Log], Theorem 2.8), Theorem 4.2 offers a quick way of computing the class of the closure of the locus $\mathcal{W}^{r+1}$ of $(r+1)$ Weierstrass points in $\overline{\mathcal{M}}_{g, 1}$ which is the main result of [CF]. 
First we determine the class of the locus $\mathfrak{M r c}_{g, i}^{r}$ over the interior $\mathcal{M}_{g, n}$. In order to do this, we first recall a few well-known intersection theory relations (see e.g. [HM]):

Lemma 4.4. If $q: \mathcal{X} \rightarrow \mathcal{M}_{g, n}$ is the morphism defined earlier, one has the following identities:

(i) $q_{*}\left(f^{*} c_{1}\left(\omega_{p}\right)^{2}\right)=12 \lambda$.

(ii) $q_{*}\left(q^{*} \lambda \cdot f^{*} c_{1}\left(\omega_{p}\right)\right)=(2 g-2) \lambda$.

(iii) $q_{*} q^{*}\left(\lambda^{2}\right)=0$.

(iv) $q_{*}\left(c_{1}\left(E_{j}\right) \cdot q^{*} \lambda\right)=\lambda$.

(v) $q_{*}\left(c_{1}\left(E_{j}\right) \cdot f^{*} c_{1}\left(\omega_{p}\right)\right)=\psi_{j}$.

(vi) $q_{*} q^{*} c_{2}\left(\pi^{*}(\mathbb{E})\right)=0$.

(vii) $q_{*}\left(c_{1}\left(E_{j}\right)^{2}\right)=-\psi_{j}$.

Proposition 4.5. If $a$ and $b$ are the numbers defined in the statement of Theorem 4.2, we have the following relation in $\operatorname{Pic}\left(\mathcal{M}_{g, n}\right)$ :

$$
\mathfrak{M r c}_{g, i}^{r} \equiv \frac{1}{g-1}\left(\begin{array}{c}
g-1 \\
i
\end{array}\right)\left(a \lambda+b \sum_{j=1}^{n} \psi_{j}\right) .
$$

Proof. Since $\phi$ is generically non-degenerate (cf. Proposition 4.1), we have the identity $\mathfrak{M r c}_{g, i}^{r} \equiv c_{1}\left(\mathcal{A}_{i-1, r+2}\right)-c_{1}\left(\pi^{*}\left(\wedge^{i} \mathbb{E}\right) \otimes \mathcal{A}_{0, r+1}\right)$. To compute these Chern classes we use Grothendieck-Riemann-Roch applied to the proper map $q$. For simplicity we set $\mathfrak{D}:=$ $\sum_{j=1}^{n} E_{j}$ and $\left.\mathcal{F}:=f^{*}\left(\wedge^{i-1} \mathcal{M} \otimes \omega_{p}^{\otimes(r+2)}\right) \otimes \mathcal{O}_{\mathcal{X}}(-\mathfrak{D})\right)$. Then we have that

$\left.c_{1}\left(\mathcal{A}_{i-1, r+2}\right)=q_{*}\left[\left(\left(\begin{array}{c}g-1 \\ i-1\end{array}\right)+c_{1}(\mathcal{F})+\frac{c_{1}^{2}(\mathcal{F})-2 c_{2}(\mathcal{F})}{2}+\cdots\right) \cdot\left(1-\frac{f^{*} c_{1}\left(\omega_{p}\right)}{2}+\frac{f^{*} c_{1}^{2}\left(\omega_{p}\right)}{12}\right)+\ldots\right)\right]$.

Using that $c_{1}(\mathcal{M})=p^{*}(\lambda)-c_{1}\left(\omega_{p}\right)$, one obtains that

$$
c_{1}(\mathcal{F})=\left(\begin{array}{c}
g-2 \\
i-2
\end{array}\right) q^{*}(\lambda)+\left((r+2)\left(\begin{array}{c}
g-1 \\
i-1
\end{array}\right)-\left(\begin{array}{c}
g-2 \\
i-2
\end{array}\right)\right) f^{*} c_{1}\left(\omega_{p}\right)-\left(\begin{array}{c}
g-1 \\
i-1
\end{array}\right) c_{1}(\mathfrak{D}) .
$$

We also use the identity

$$
\begin{aligned}
c_{2}(\mathcal{F})=c_{2}\left(f^{*}\right. & \left.\wedge^{i-1} \mathcal{M}\right)+\left(\left(\begin{array}{c}
g-1 \\
i-1
\end{array}\right)-1\right) c_{1}\left(f^{*} \wedge^{i-1} \mathcal{M}\right) \cdot\left((r+2) f^{*} c_{1}\left(\omega_{p}\right)-c_{1}(\mathfrak{D})\right)+ \\
& +\frac{1}{2}\left(\begin{array}{c}
g-1 \\
i-1
\end{array}\right)\left(\left(\begin{array}{c}
g-1 \\
i-1
\end{array}\right)-1\right)\left((r+2) f^{*} c_{1}\left(\omega_{p}\right)-c_{1}(\mathfrak{D})\right)^{2},
\end{aligned}
$$

which together with the formula $c_{2}(\mathcal{M})=c_{1}^{2}\left(\omega_{p}\right)-c_{1}\left(\omega_{p}\right) \cdot p^{*}(\lambda)$ and Lemma 4.4, enable us to compute $c_{1}\left(\mathcal{A}_{i-1, r+2}\right)$. In a similar fashion, we obtain from GrothendieckRiemann-Roch applied to the map $q$, that

$$
c_{1}\left(\mathcal{A}_{0, r+1}\right)=\left(6 r^{2}+6 r+1\right) \lambda-(r+1) \sum_{j=1}^{n} \psi_{j}
$$

and finally

$$
c_{1}\left(\pi^{*} \wedge^{i} \mathbb{E} \otimes \mathcal{A}_{0, r+1}\right)=\left(\begin{array}{c}
g \\
i
\end{array}\right) c_{1}\left(\mathcal{A}_{0, r+1}\right)+((2 r+1)(g-1)-r)\left(\begin{array}{c}
g-1 \\
i-1
\end{array}\right) \lambda
$$


which quickly leads to the stated formula.

To compute the remaining coefficients in $\left[\overline{\mathfrak{M r c}}_{g, i}^{r}\right]$ we extend the vector bundles $\mathcal{A}_{a, b}$ to sheaves over $\overline{\mathcal{M}}_{g, n}$ as follows. We denote by $q: \overline{\mathcal{M}}_{g, n+1} \rightarrow \overline{\mathcal{M}}_{g, n}$ the projection dropping the $(n+1)$-st marked point and by $\pi: \overline{\mathcal{M}}_{g, n} \rightarrow \overline{\mathcal{M}}_{g}$ the forgetful map. We introduce the following twist of the Hodge bundle on $\overline{\mathcal{M}}_{g, n}$ :

$$
\mathcal{H}:=q_{*}\left(\omega_{q} \otimes \mathcal{O}_{\overline{\mathcal{M}}_{g, n+1}}\left(\sum_{[g / 2] \leq j \leq g-1} \sum_{|S| \leq n}(g-j) \Delta_{j: S}\right)\right) .
$$

(In other words, the fibre of $\mathcal{H}$ over a pointed curve from $\pi^{*}\left(\Delta_{j}\right)$ where $[g / 2] \leq j \leq g$, is the space of global sections of the genus $j$-aspect of the limit $\mathfrak{g}_{2 g-2}^{g-1}$ corresponding to the canonical linear series). We then define

$$
\mathcal{M}:=\operatorname{Ker}\left\{q^{*}(\mathcal{H}) \rightarrow \mathcal{O}_{\overline{\mathcal{M}}_{g, n+1}}\left(\sum_{[g / 2] \leq j \leq g-1} \sum_{|S| \leq n}(g-j) \Delta_{j: s}\right)\right\} .
$$

Furthermore, for each pair of integers $0 \leq a \leq i, b \geq r+2$ or $(a, b)=(0, r+1)$, we define

$$
\mathcal{A}_{a, b}:=q_{*}\left(\wedge^{a} \mathcal{M} \otimes \omega_{q}^{\otimes b} \otimes \mathcal{O}_{\overline{\mathcal{M}}_{g, n+1}}\left(\sum_{[g / 2] \leq j \leq g-1} \sum_{|S| \leq n}((2 b-1)(g-j)-b) \Delta_{j: S}-\sum_{j=1}^{n} \Delta_{0: j, n+1}\right)\right) .
$$

(Obviously, this is an extension of the definition of $\mathcal{A}_{a, b}$ over $\mathcal{M}_{g, n}$ ). The twists were chosen in such a way that we have exact sequences of the type

$$
0 \longrightarrow \mathcal{A}_{a, b} \longrightarrow \wedge^{a} \mathcal{H} \otimes \mathcal{A}_{0, b} \longrightarrow \mathcal{A}_{a-1, b+1} \longrightarrow 0,
$$

at least in a dense open subset inside $\pi^{-1}\left(\mathcal{M}_{g} \cup \Delta_{0} \cup \Delta_{1}\right)$. Also, there exists a morphism $\phi: \wedge^{i} \mathcal{H} \otimes \mathcal{A}_{0, r+1} \rightarrow \mathcal{A}_{i-1, r+2}$ which over $\mathcal{M}_{g, n}$ restricts to the vector bundle map defined in Proposition 4.1 .

Proof of Theorem 4.2. We expand the class of $\overline{\mathfrak{M r c}}_{g, i}^{r}$ in $\operatorname{Pic}\left(\overline{\mathcal{M}}_{g, n}\right)$ :

$$
\overline{\mathfrak{M r c}}_{g: i}^{r} \equiv A \lambda+B \sum_{j=1}^{n} \psi_{j}-B_{i r r} \delta_{i r r}-\sum_{j, s \geq 0} B_{j: s} \sum_{|S|=j} \delta_{j: S}
$$

We have already determined the values of $A$ and $B$. One can write down the following relation in $\operatorname{Pic}\left(\overline{\mathcal{M}}_{g, n}\right)$ :

$$
c_{1}\left(\mathcal{A}_{i-1, r+2}-\wedge^{i} \mathcal{H} \otimes \mathcal{A}_{0, r+1}\right)=\left[\overline{\mathfrak{M r c}}_{g, i}^{r}\right]+\sum_{j, s \geq 0} d_{j, s} \sum_{|S|=s} \delta_{j: S},
$$

where $d_{j, s}$ is the multiplicity of the divisor $\Delta_{j: S}$ in the degeneracy locus of $\phi$. By intersecting both sides of (9) with test curves in $\overline{\mathcal{M}}_{g, n}$, sometimes we are able to show that $\phi$ is generically non-degenerate along $\Delta_{j: S}$ (that is, $d_{j: s}=0$ ), and then we explicitly determine the value of $B_{j: S}$ in Theorem 4.2, otherwise we only get lower bounds on $B_{j: S}$. We are only going to explain in detail the case of the coefficient $B_{i r r}$ the remaining ones being somewhat similar.

We define a test curve in the boundary of $\overline{\mathcal{M}}_{g, n}$ as follows. If $\left[C, q, x_{1}, \ldots, x_{n}\right] \in$ $\mathcal{M}_{g-1, n+1}$ is a general pointed curve, then we define the 1-dimensional family

$$
C_{n}^{0}:=\left\{\left[C / y \sim q, x_{1}, \ldots, x_{n}\right]\right\}_{y \in C} \subset \Delta_{i r r} \subset \overline{\mathcal{M}}_{g, n} .
$$


The fibre of this family when the variable point $y \in C$ hits the fixed marked point $x_{i}$ for $1 \leq i \leq n$ is the pointed curve $\left(\tilde{C}_{x_{i}}:=C \cup_{x_{1}, q} \mathbf{P}^{1}, \tilde{x}_{1}, x_{2}, \ldots, x_{n}\right)$, where $\tilde{x}_{1} \in \mathbf{P}^{1}$ (here we regard $x_{1}, \tilde{x}_{1}, q \in \mathbf{P}^{1}$ as being three distinct points). One has the identities

$C_{n}^{0} \cdot \delta_{i r r}=-2 g+2, C_{n}^{0} \cdot \delta_{1: \emptyset}=1, C_{n}^{0} \cdot \psi_{i}=1$ for $1 \leq i \leq n, C_{n}^{0} \cdot \lambda=C_{n}^{0} \cdot \delta_{i: S}=0$ for $(i, S) \neq(1, \emptyset)$.

By intersecting both sides of (9) with $C_{n}^{0}$ one can write down the identity $C_{n}^{0} \cdot \overline{\mathfrak{M r c}}_{g, i}^{r}=$ $(2 g-2) B_{i r r}+n B-B_{1: \emptyset}$. On the other hand one also has the relation

$$
A-12 B_{\text {irr }}+B_{1: \emptyset}=0
$$

reflecting the fact that $\overline{\mathfrak{M r c}}_{g, i}^{r}$ is physically disjoint from the curve $\left\{\left[C \cup_{q} R, x_{1}, x_{2}, \ldots, x_{n}\right]\right\}_{R}$ obtained by attaching to a fixed Brill-Noether general curve $\left[C, q, x_{1}, \ldots, x_{n}\right] \in \mathcal{M}_{g-1, n+1}$ a pencil of plane cubics in which $R$ denotes a generic member. Thus determining $B_{i r r}$ and $B_{1: \emptyset}$ boils down to (i) showing that $\phi$ is generically non-degenerate along $C_{n}^{0}$ and (ii) estimating the intersection number $C_{n}^{0} \cdot c_{1}\left(\mathcal{A}_{i-1, r+2}-\wedge^{i} \mathcal{H} \otimes \mathcal{A}_{0, r+1}\right)$. By local analysis one can see that for $1 \leq l \leq i-1$ there are exact sequences of bundles over $C_{n}^{0}$

$$
0 \longrightarrow \mathcal{A}_{i-l, r+l+1 \mid C_{n}^{0}} \longrightarrow \wedge^{i-l} \mathcal{H} \otimes \mathcal{A}_{0, r+l+1 \mid C_{n}^{0}} \longrightarrow \mathcal{A}_{i-l-1, r+l+2 \mid C_{n}^{0}} \longrightarrow 0,
$$

therefore we can write the identities

$$
\begin{gathered}
C_{n}^{0} \cdot c_{1}\left(\mathcal{A}_{i-1, r+2}\right)=\sum_{l=1}^{i}(-1)^{l-1} c_{1}\left(\wedge^{i-l} \mathcal{H}_{\mid C_{n}^{0}} \otimes \mathcal{A}_{0, r+l+1 \mid C_{n}^{0}}\right)= \\
\sum_{l=1}^{i}(-1)^{l-1}\left(\left(\begin{array}{c}
g-1 \\
i-l-1
\end{array}\right)((2 r+2 l+1)(g-1)-n) c_{1}\left(\mathcal{H}_{\mid C_{n}^{0}}\right)+\left(\begin{array}{c}
g \\
i-l
\end{array}\right) c_{1}\left(\mathcal{A}_{0, r+l+1 \mid C_{n}^{0}}\right)\right) .
\end{gathered}
$$

Next we describe the vector bundle $\mathcal{A}_{0, j \mid C_{n}^{0}}$. We identify $C_{n}^{0}$ with $C$ via the map

$$
C \ni y \mapsto\left[C / y \sim q, x_{1}, \ldots, x_{n}\right] \in \overline{\mathcal{M}}_{g, n}
$$

and denote by $p_{1}, p_{2}: C \times C \rightarrow C$ the two projections, by $\Delta \subset C \times C$ the diagonal and set $\Gamma_{q}:=\{q\} \times C \subset C \times C$. Then for every $j \geq r$ we have the following exact sequence of vector bundles on $C$ :

$$
\begin{gathered}
0 \longrightarrow\left(p_{2}\right)_{*}\left(p_{1}^{*}\left(K_{C}^{\otimes j}\right) \otimes \mathcal{O}_{C \times C}\left((j-1) \Delta+(j-1) \Gamma_{q}-\sum_{j=1}^{n}\left\{x_{j}\right\} \times C\right)\right) \longrightarrow \mathcal{A}_{0, j \mid C_{n}^{0}} \longrightarrow \\
\longrightarrow\left(p_{2}\right)_{*}\left(p_{1}^{*}\left(K_{C}^{\otimes j}\right) \otimes \mathcal{O}_{C \times C}\left(j \Delta+j \Gamma_{q}-\sum_{j=1}^{n}\left\{x_{j}\right\} \times C\right) \otimes \mathcal{O}_{\Gamma_{q}}\right) \otimes \mathcal{O}_{C}\left(-x_{1}-\cdots-x_{n}\right) \longrightarrow 0,
\end{gathered}
$$

which quickly leads to the formula

$$
c_{1}\left(\mathcal{A}_{0, j \mid C_{n}^{0}}\right)=1+2 j-2 j r g-2 j g-j^{2}+j^{2} g+2 j r+2 j i .
$$

Since one also has that $c_{1}\left(\mathcal{H}_{\mid C_{n}^{0}}\right)=1$ (use that $\mathcal{H}(y)=H^{0}\left(K_{C} \otimes \mathcal{O}_{C}(y+q)\right.$ ) for each $y \in C_{n}^{0}$ under the identification described above), we obtain a formula for $C_{n}^{0} \cdot \overline{\mathfrak{M r c}}_{g, i}^{r}$ and ultimately a formula for $B_{i r r}$. Dealing with the other coefficients $B_{j: S}$ is similar in general. 


\subsection{Divisors defined by imposing linear conditions on marked points.}

Here we present another general construction that produces families of effective divisors on $\overline{\mathcal{M}}_{g, n}$. Like in Section 2, we pick integers $g, r, d \geq 1$ such that $\rho(g, r, d)=0$, therefore we can write $d=r s+r$ and $g=r s+s$ for some integer $s \geq 1$. We set $n:=r+1$ and define the following divisor on $\mathcal{M}_{g, n}$ :

$\mathfrak{L i n}_{d}^{r}:=\left\{\left[C, x_{1}, \ldots, x_{r+1}\right]: \exists L \in W_{d}^{r}(C)\right.$ such that $\left.h^{0}\left(L \otimes \mathcal{O}_{C}\left(-x_{1}-\cdots-x_{r+1}\right)\right) \geq 1\right\}$.

We recall that we have introduced the number

$$
N:=c_{r}=g ! \frac{1 ! 2 ! \ldots r !}{(g-d+r) ! \ldots(g-d+2 r) !},
$$

which counts linear series $\mathfrak{g}_{d}^{r}$ on a general curve of genus $g$ (cf. [ACGH]). Our main result is the computation of the class $\left[\overline{\mathfrak{L i n}}_{d}^{r}\right]$ :

Theorem 4.6. Fix integers $r, s \geq 1$ and set $d:=r s+r, g:=r s+s$. Then $\overline{\mathfrak{L i n}}_{d}^{r}$ is an effective divisor on $\overline{\mathcal{M}}_{g, n}$ and we have the following formula for its class in $\operatorname{Pic}\left(\overline{\mathcal{M}}_{g, r+1}\right)$ :

$$
\overline{\mathfrak{L i n}}_{d}^{r} \equiv \frac{r c_{r}}{r s+s-1}\left(a \lambda+c \sum_{j=1}^{r+1} \psi_{j}-b_{i r r} \delta_{i r r}-\sum_{j, t \geq 0} b_{j: t} \sum_{|S|=t} \delta_{j: S}\right),
$$

where

$$
\begin{gathered}
a=\frac{(r+2)\left(r^{2} s^{3}-r^{2} s+2 r s^{3}+6 r s^{2}-2 r s-8 r+s^{3}+6 s^{2}+3 s-8\right)}{2(s+r+1)(r s+s-2)}, \\
c=\frac{s+1}{2}, b_{i r r}=\frac{(s-1)(s+1)(r+1)(r+2)(r s+s+4)}{12(s+r+1)(r s+s-2)}, \\
b_{j: 0}=\frac{j(r+2)\left(r s\left(s^{2}-1\right)(r+2)+s\left(s^{2}-2 j-3\right)+(r+1)\left(3 s^{2}-j s^{2}+2 j-2\right)\right)}{2(r+s+1)(r s+s-2)} \text { for } j \geq 1 \\
b_{1: t}=\left(\begin{array}{c}
t-1 \\
2
\end{array}\right) \frac{r s+s-1}{r}+\frac{(s-1)(s+1)(r+1)\left(r^{3} s+3 r^{2} s-2 s+4\right)}{2 r(r+s+1)(r s+s-2)} \text { for all } t \geq 1 \\
\text { and } b_{j: t} \geq b_{0: t} \text { for all } j \geq 2 .
\end{gathered}
$$

Remark 4.7. For $s=1$ and $r=g-1$, Theorem 4.6 specializes to Logan's formula for the class of the divisor $\overline{\mathfrak{L i n}}_{2 g-2}^{g-1}$ of points $\left[C, x_{1}, \ldots, x_{g}\right] \in \mathcal{M}_{g, g}$ with $h^{0}\left(\mathcal{O}_{C}\left(x_{1}+\cdots+x_{g}\right)\right) \geq$ 2. We have the formula (cf. [Log], Theorem 5.4):

$$
\overline{\mathfrak{L i n}}_{2 g-2}^{g-1} \equiv-\lambda+\sum_{j=1}^{g} \psi_{j}-0 \cdot \delta_{i r r}-\sum_{t=2}^{g}\left(\begin{array}{c}
t+1 \\
2
\end{array}\right) \sum_{|S|=t} \delta_{0: S}-\sum_{t=1}^{g}\left(\begin{array}{l}
t \\
2
\end{array}\right) \sum_{|S|=t} \delta_{1: S}-\cdots
$$

In the next case, $s=2, g=2 r+2$ and $d=3 r$ we get a new divisor on $\overline{\mathcal{M}}_{2 r+2, r+1}$ and our formula reads

$$
\overline{\mathfrak{L i n}}_{3 r}^{r} \equiv \frac{1}{2(2 r+1)}\left((3 r+5)(r+2) \lambda+3 r \sum_{j=1}^{r+1} \psi_{j}-\left(\begin{array}{c}
r+2 \\
2
\end{array}\right) \delta_{i r r}-\sum_{|S|=2} \delta_{0: S}-\cdots\right)
$$


Proof of Theorem 4.6 Proving that $\mathfrak{L i n}_{d}^{r}$ is a divisor on $\mathcal{M}_{g, r+1}$ follows immediately from Brill-Noether theory: a general $[C] \in \mathcal{M}_{g}$ has precisely $N$ linear series $L \in W_{d}^{r}(C)$ and each of them is base point free and satisfies $h^{0}(L)=r+1$ and $\mathfrak{L i n}_{d}^{r}$ consists of those $(r+1)$-tuples of points on $C$ which are not in general linear position with respect to some $L \in W_{d}^{r}(C)$. We start the calculation of the class of $\overline{\mathfrak{L i n}}_{d}^{r}$ by determining the coefficients of $\lambda, \delta_{i r r}$ and $\psi_{j}$. To do this we use the observation that if $\pi_{n}: \overline{\mathcal{M}}_{g, n} \rightarrow \overline{\mathcal{M}}_{g, n-1}$ is the projection forgetting the marked point labeled by $n$ and $D$ is any divisor class on $\overline{\mathcal{M}}_{g, n}$, then for distinct labels $i, j \neq n$, the $\lambda, \delta_{i r r}$ and $\psi_{j}$ coefficients of the divisors $D$ on $\overline{\mathcal{M}}_{g, n}$ and $\left(\pi_{n}\right)_{*}\left(D \cdot \delta_{0: i n}\right)$ on $\overline{\mathcal{M}}_{g, n-1}$ are the same (see [FMP], Prop. 4.4). The divisor $\left(\pi_{n}\right)_{*}\left(D \cdot \delta_{0: \text { in }}\right)$ can be thought of as the locus of those points $\left[C, x_{1}, \ldots, x_{n}\right] \in D$ where the points $x_{i}$ and $x_{n}$ are allowed to come together. Using this observation repeatedly, we obtain that the divisor $\overline{\mathfrak{L i n}}_{d}^{r}(1)$ on $\overline{\mathcal{M}}_{g, 1}$ obtained by letting all points $x_{1}, \ldots, x_{n}$ come together, has the same $\lambda$ and $\delta_{i r r}$ coefficients as $\mathfrak{L i n}_{d}^{r}$. But clearly

$$
\mathfrak{L i n}_{d}^{r}(1)=\left\{[C, x] \in \mathcal{M}_{g, 1}: \exists L \in W_{d}^{r}(C) \text { such that } h^{0}\left(L \otimes \mathcal{O}_{C}(-(r+1) x)\right) \geq 1\right\},
$$

that is, $\mathfrak{L i n}_{d}^{r}(1)$ is generically the locus of ramification points in one of the finitely many linear series $\mathfrak{g}_{d}^{r}$ on a given curve of genus $g$. By applying Theorem 4.1 from [EH2], we obtain that the class of $\overline{\mathfrak{L i n}}_{d}^{r}(1)$ can be written as a combination $\overline{\mathfrak{L i n}}_{d}^{r}(1) \equiv \mu \cdot B N+\nu \cdot \mathcal{W}$, where

$$
B N:=(g+3) \lambda-\frac{g+1}{6} \delta_{i r r}-\sum_{j=1}^{g-1} j(g-j) \delta_{j: 1}
$$

is the pull-back from $\overline{\mathcal{M}}_{g}$ of the class of the Brill-Noether divisor and

$$
\mathcal{W}:=-\lambda+\left(\begin{array}{c}
g+1 \\
2
\end{array}\right) \psi-\sum_{j=1}^{g-1}\left(\begin{array}{c}
g-j+1 \\
2
\end{array}\right) \delta_{j: 1}
$$

is the class of the Weierstrass divisor. To determine the coefficients $\mu$ and $\nu$ we use two test curves inside $\overline{\mathcal{M}}_{g, 1}$. First we fix a genus $g$ curve $C$ and we let the marked point vary along $C$. If we denote this curve by $\bar{C} \subset \overline{\mathcal{M}}_{g, 1}$, then the only generator of $\operatorname{Pic}\left(\overline{\mathcal{M}}_{g, 1}\right)$ which has non-zero intersection number with $\bar{C}$ is $\psi$, and $\bar{C} \cdot \psi=2 g-2$. On the other hand $\bar{C} \cdot \overline{\mathfrak{L i n}}_{d}^{r}(1)$ is the total number of ramification points on all $\mathfrak{g}_{d}^{r \text { 's on }}$ $C$. This number is $N(r+1)(d+r(g-1)$ ) (see e.g. [EH1], pg. 345), which shows that $\nu=N(r+1)(d+r(g-1)) /((g-1) g(g+1))$. To compute $\mu$ we use a second test curve constructed as follows: we fix a 2-pointed elliptic curve $[E, x, y] \in \mathcal{M}_{1,2}$ such that the class $x-y \in \operatorname{Pic}^{0}(E)$ is not torsion, and a fixed general curve $[C] \in \mathcal{M}_{g-1}$. We define the family $\bar{C}_{1}:=\left\{\left(C \cup_{y} E, x\right)\right\}_{y \in C}$ (that is, the point of attachment varies on $C)$. The only generator of $\operatorname{Pic}\left(\overline{\mathcal{M}}_{g, 1}\right)$ meeting $\bar{C}_{1}$ non-trivially is $\delta_{1: 1}=\delta_{g-1: \emptyset}$, in which case $\bar{C}_{1} \cdot \delta_{1 ; 1}=-2 g+4$. The calculation of $\bar{C}_{1} \cdot \overline{\mathfrak{L i n}}_{d}^{r}(1)$ is a standard exercise in the theory of limit linear series. Suppose $l=\left\{l_{C}, l_{E}\right\}$ is a limit $\mathfrak{g}_{d}^{r}$ on $C \cup_{y} E$ such that $a_{r}^{l_{E}}(x) \geq r+1$. Then because the class $x-y \in \operatorname{Pic}^{0}(E)$ is not torsion, we must have that $\rho\left(l_{E}, x, y\right)=0$ and $\rho\left(l_{C}, y\right)=-1$. An easy calculation shows that we must also have $a^{l_{E}}(x)=(0,1, \ldots, r-1, r+1)$ and $a^{l_{C}}(y)=(0,2,3, \ldots, r, r+2)$, and moreover the aspect $l_{E}$ is uniquely determined. Thus we have to count the number of points $y \in C$ such that there exists $L \in W_{d}^{r}(C)$ with the property that $h^{0}(L(-2 y)) \geq r$ and $h^{0}(L(-(r+2) y)) \geq 1$. 
To compute this number we further degenerate the curve $C$ to a transverse union $R \cup_{y_{1}} E_{1} \cup \ldots \cup_{y_{g-1}} E_{g-1}$ consisting of a smooth rational spine $R$ and $g-1$ elliptic tails $E_{1}, \ldots, E_{g-1}$. Using Proposition 1.1 from [EH1] we see that the point $y$ has to specialize to one of the tails $E_{j}$, and without loss of generality we assume that $y \in E_{1}$ (all the intersection numbers we compute will be multiplied by $g-1$ to account for $y$ lying on a different elliptic tail). Suppose now that $l=\left\{l_{R}, l_{E_{1}}, \ldots, l_{E_{g-1}}\right\}$ is a limit $\mathfrak{g}_{d}^{r}$ on $R \cup E_{1} \cup \ldots \cup E_{g-1}$ such that $a^{l_{E_{1}}}(y)=(0,2,3, \ldots, r, r+2)$. Then $\rho\left(l_{R}, y_{1}, \ldots, y_{g-1}\right)=$ $0, \rho\left(l_{E_{j}}, x_{j}\right)=0$ for $2 \leq j \leq g-1$ and $\rho\left(l_{E_{1}}, y_{1}, y\right)=-1$. A close inspection shows that there are three numerical possibilities:

$(\alpha) a^{l_{R}}\left(y_{1}\right)=(0,2,4,5, \ldots, r, r+1, r+3)$ and then $y_{1}-y \in \operatorname{Pic}^{0}\left(E_{1}\right)[2]$. This contribution will be equal to $3(g-1)$ multiplied by the number of $\mathfrak{g}_{d}^{r \prime}$ s on $R$ having ordinary cusps at $g-2$ general points and vanishing $(0,2,4, \ldots, r+1, r+3)$ at another fixed point. By Schubert calculus this number equals the product of Schubert cycles $\sigma_{(0,1, \ldots, 1)}^{g-2} \cdot \sigma_{(0,1,2, \ldots, 2,3)} \in H^{*}(\mathbf{G}(r, d))$.

( $\beta$ ) $a^{l_{R}}\left(y_{1}\right)=(0,3,4, \ldots, r+1, r+2)$, in which case $y-y_{1} \in \operatorname{Pic}^{0}\left(E_{1}\right)[r+2]$. The number we get in this situation is $(g-1)\left((r+2)^{2}-1\right) \sigma_{(0,1, \ldots, 1)}^{g-2} \cdot \sigma_{(0,2, \ldots, 2)} \in H^{*}(\mathbf{G}(r, d))$.

$(\gamma) a^{l_{r}}(y)=(1,2,4, \ldots, r+1, r+2)$ and then $y-y_{1} \in \operatorname{Pic}^{0}\left(E_{1}\right)[r]$. We obtain a final contribution of $(g-1)\left(r^{2}-1\right) \sigma_{(0,1, \ldots, 1)}^{g-2} \cdot \sigma_{(0,0,1, \ldots, 1)} \in H^{*}(\mathbf{G}(r, d-1))$. Adding all these together and using (3), we obtain that the total intersection number is

$$
\bar{C}_{1} \cdot \overline{\mathfrak{L i n}}_{d}^{r}(1)=\frac{N r(r+1)(r+2)\left(r s+2 s^{2}-4+s\right)}{s+r+1},
$$

which leads to

$$
\mu=\frac{N r(r+1)(r+2)(s-1)(s+1)(r s+s+4)}{2(s+r+1)(r s+s-2)(r s+s-1)(r s+s+1)}
$$

and then the stated formulas for the $\lambda$ and $\delta_{i r r}$ coefficients. We also note that the $\delta_{j: \emptyset}$ coefficient of $\overline{\operatorname{Lin}}_{d}^{r}$ equals the $\delta_{j: \emptyset}=\delta_{g-j: 1}$ coefficient of $\overline{\mathfrak{L i n}}_{d}^{r}(1)$ and this is equal to $j(g-j) \mu+j(j+1) \nu / 2$ and we obtain the desired expression for $b_{j: 0}$. Next we determine the coefficient $c$ of the $\psi_{j}$ classes. We introduce the divisor $\overline{\mathfrak{L i n}}_{d}^{r}(2)$ on $\overline{\mathcal{M}}_{g, 2}$ obtained by letting $x_{2}, \ldots, x_{r+1}$ coincide while keeping $x_{1}$ apart. Then

$$
\mathfrak{L i n}_{d}^{r}(2)=\left\{\left[C, x_{1}, x_{2}\right] \in \mathcal{M}_{g, 2}: \exists L \in W_{d}^{r}(C) \text { such that } h^{0}\left(L \otimes \mathcal{O}_{C}\left(-x_{1}-r x_{2}\right)\right) \geq 1\right\}
$$

and we can write $\overline{\mathfrak{L i n}}_{d}^{r}(2) \equiv N\left(a \lambda+c \psi_{1}+c_{2} \psi_{2}-e_{12} \delta_{0: 12}-\cdots\right)$ (that is, the $\lambda$ and $\psi_{1}$ coefficients coincide with those of $\overline{\mathfrak{L i n}}_{d}^{r}$. We intersect $\overline{\mathfrak{L i n}}_{d}^{r}(2)$ with two curves in $\overline{\mathcal{M}}_{g, 2}$ : consider a curve $C$ of genus $g$ and define $\tilde{C}_{1}:=\left\{\left[C, x_{1}, x_{2}\right]\right\}_{\left\{x_{2} \text { moves on } C\right\}}$ and

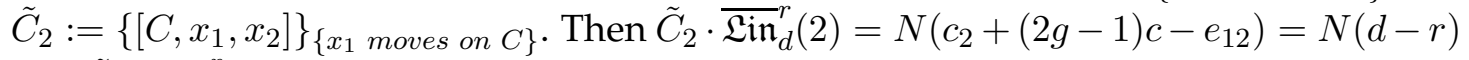
and $\tilde{C}_{1} \cdot \overline{\mathfrak{L i n}}_{d}^{r}(2)=N\left((2 g-1) c_{2}+c-e_{12}\right)=N(r+1)(d-1+(r-1)(g-1))$. (The first identity is obvious, for the second, use that what we are counting is the total number of ramification points on all linear series $L \otimes \mathcal{O}_{C}\left(-x_{1}\right)$, where $L \in W_{d}^{r}(C)$ and $x_{1} \in C$ is a fixed general point). We thus have a system of two equations in the unknowns $c, c_{2}$ and $e_{12}$, but we can also use that $e_{12}$ equals the $\psi$ coefficient of $\overline{\mathfrak{L i n}}_{d}^{r}(1)=\left(\pi_{2}\right)_{*}\left(\overline{\mathfrak{L i n}}_{d}^{r}(2) \cdot \delta_{0: 12}\right)$, where $\pi_{2}: \overline{\mathcal{M}}_{g, 2} \rightarrow \overline{\mathcal{M}}_{g, 1}$ is the map forgetting the second point. Thus $e_{12}=\nu g(g+$ 
$1) / 2$, which gives us enough relations to determine $c$. We note that in this way we also determine $b_{0: 2}=(2 r s+r+s-1) /(r s+s-1)$.

To compute the coefficient $b_{j: t}$ for $1 \leq t \leq r+1$ we consider another test curve defined as follows: we fix integers $1 \leq j \leq g-1$ and $1 \leq t \leq r+1$, together with general pointed curves $\left[C, y, x_{2}, \ldots, x_{t}\right] \in \mathcal{M}_{j, t}$ and $\left[Y, y, x_{t+1}, \ldots, x_{r+1}\right] \in \mathcal{M}_{g-j, r-t+2}$. We define the test curve $\bar{C}_{j, s}:=\left\{C \cup_{y} Y, x_{1}, \ldots, x_{t}, x_{t+1}, x_{r+1}\right\}_{x_{1} \in C}$ (thus $x_{1}$ is the moving point on the genus $j$ component). Then we have the relation

$$
\bar{C}_{j, t} \cdot \overline{\mathfrak{L i n}}_{d}^{r}=(2 j+2 t-3) c-(t-1) b_{0: 2}+b_{j: t}-b_{j: t-1},
$$

which can be used to compute $b_{j: t}$ provided we know $b_{j: t-1}$ (note that we have already computed $b_{j: \emptyset}$ for all $j$ ).

We now describe directly the intersection cycle $\bar{C}_{j, t} \cdot \overline{\mathfrak{L i n}}_{d}^{r}$. Since $[C, y] \in \mathcal{M}_{j, 1}$ and $[Y, y] \in \mathcal{M}_{g-j, 1}$ are general, on the stable $Y \cup_{y} C$ there will be precisely $N$ limit $\mathfrak{g}_{d}^{r \prime} \mathrm{s}$ which can be described as follows: We first choose a Schubert (ramification) sequence $\max \{0, j-t\} \leq \alpha_{0} \leq \ldots \alpha_{r} \leq j$ such that $\sum_{i=0}^{r} \alpha_{i}=r j$. Then we choose $l_{Y} \in G_{d}^{r}(Y)$ having vanishing sequence $\left(a^{l_{Y}}(y)=\alpha_{i}+i\right)_{0 \leq i \leq r}$; in fact there will be $\sigma_{(0,1, \ldots, 1)}^{g-j} \cdot \sigma_{\left(\alpha_{0}, \ldots, \alpha_{r}\right)} \in H^{*}(\mathbf{G}(r, d))$ such linear series. On $C$ we choose a complementary linear series $l_{C} \in G_{d}^{r}(C)$ with vanishing sequence $\left(a_{i}^{l_{C}}(y)=r s+i-\alpha_{i}\right)_{0 \leq i \leq r}$; there are $\sigma_{(0,1, \ldots, 1)}^{j} \cdot \sigma_{\left(r s-\alpha_{r}, \ldots, r s-\alpha_{0}\right)} \in H^{*}(\mathbf{G}(r, d))$ choices. Every limit linear series on $C \cup_{y} Y$ appears in this way and the intersection $\bar{C}_{j, t} \cdot \overline{\mathfrak{L i n}}_{d}^{r}$ is everywhere transverse (cf. [EH3]). We also have the identity

$$
N=\sum_{j-t \leq \alpha_{0} \leq \ldots \leq \alpha_{r} \leq j, \sum_{l=0}^{r} \alpha_{l}=j}\left(\sigma_{(0,1, \ldots, 1)}^{g-j} \cdot \sigma_{\left(\alpha_{0}, \ldots, \alpha_{r}\right)}\right)\left(\sigma_{(0,1, \ldots, 1)}^{j} \cdot \sigma_{\left(r s-\alpha_{r}, \ldots, r s-\alpha_{0}\right)}\right) .
$$

If $l=\left\{l_{C}, l_{Y}\right\}$ is one of these $N$ limit $\mathfrak{g}_{d}^{r \prime}$ s corresponding to a sequence $\left(\alpha_{0} \leq \ldots \leq \alpha_{r}\right)$ as above, the condition that there exists $x_{1} \in C$ such that the divisor $x_{1}+\cdots+x_{r+1}$ is the specialization of a linear divisor with respect to a $\mathfrak{g}_{d}^{r}$ on a nearby smooth curve, can be translated as follows: there exist sections $\sigma_{Y} \in\left|l_{Y}\right|, \sigma_{C} \in\left|l_{C}\right|$ such that $\operatorname{div}\left(\sigma_{Y}\right) \geq$ $x_{t+1}+\cdots+x_{r+1}$ and $\operatorname{div}\left(\sigma_{C}\right) \geq x_{1}+\ldots+x_{t}$; the sections $\sigma_{C}$ and $\sigma_{Y}$ being the limit linear series specializations of a single section on a nearby smooth curve, they must also satisfy the compatibility relation ord $\operatorname{or}_{y}\left(\sigma_{Y}\right)+\operatorname{ord}_{y}\left(\sigma_{C}\right)=r s+r$. Because the fixed points $x_{t+1}, \ldots, x_{r+1} \in Y$ are general, they impose independent conditions on $l_{Y}$ which quickly leads to the equalities $\operatorname{ord}_{y}\left(\sigma_{Y}\right)=a_{t-1}^{l_{Y}}(y)=\alpha_{t-1}+t-1$, hence $\operatorname{ord}_{y}\left(\sigma_{C}\right)=$ $a_{r-t+1}^{l_{C}}(y)$. Thus $\operatorname{div}\left(\sigma_{C}\right) \geq a_{r-t+1}^{l_{C}}(y)+x_{2}+\ldots+x_{b}$ and up to multiplication by scalars, the sections $\sigma_{C}$ and $\sigma_{Y}$ are unique with this property. For each $\sigma_{C}$ we have precisely $d-a_{r-t+1}^{l_{C}}(y)-(t-1)=\alpha_{t-1}$ choices for $x_{1} \in C$. Therefore

$$
\bar{C}_{j, t} \cdot \overline{\mathfrak{L i n}}_{d}^{r}=\sum_{\alpha_{0} \leq \cdots \leq \alpha_{r}} \alpha_{t-1}\left(\sigma_{(0,1, \ldots, 1)}^{g-j} \cdot \sigma_{\left(\alpha_{0}, \ldots, \alpha_{r}\right)}\right) \cdot\left(\sigma_{(0,1, \ldots, 1)}^{j} \cdot \sigma_{\left(r s-\alpha_{r}, \ldots, r s-\alpha_{0}\right)}\right) .
$$

For $j=0$ the only sequence $\left(\alpha_{l}\right)_{0 \leq l \leq r}$ allowed is the sequence $(0, \ldots, 0)$ which shows that $\bar{C}_{0: t} \cdot \overline{\mathfrak{L i n}}_{d}^{r}=0$ for all $3 \leq t \leq r+1$. Since $b_{0: 2}$ has already been determined, applying (11) we obtain the stated formulas for $b_{0: t}$. Similarly, for $j=1$ the only sequence allowed is $(0,1, \ldots, 1)$ and then $\bar{C}_{1: t} \cdot \overline{\mathfrak{L i n}}_{d}^{r}=N$ for $t \geq 2$, while $\bar{C}_{1: 1} \cdot \overline{\mathfrak{L i n}}_{d}^{r}=0$; this allows us to determine $b_{1: t}$ for all $t$. When $j \geq 2$ for each sequence $\left(\alpha_{l}\right)_{0 \leq l \leq r}$ appearing in this sum, 
we have the inequalities $r j=\sum_{l=0}^{r} \alpha_{l} \leq t \alpha_{t-1}+(r+1-t) j$, therefore $\alpha_{t-1} \geq(t-1) j / t$ and then $\bar{C}_{j, t} \cdot \overline{\mathfrak{L i n}}_{d}^{r} \geq N(t-1) j / t$. To obtain the desired bound on $b_{j: t}$ we use repeatedly (11) and we can write

$$
b_{j: t}-b_{j: 0}=\left(\begin{array}{l}
t \\
2
\end{array}\right) b_{0: 2}-\left(2 b j+b^{2}-2 b\right) c+\sum_{l=1}^{t} \bar{C}_{j, l} \cdot \overline{\mathfrak{L} i \mathfrak{n}}_{d}^{r}
$$

Using the previous inequality we can now check that $b_{j: t} \geq b_{j: 0}$.

\subsection{The divisor of $n$-fold points.}

We describe another way of constructing effective divisors on $\overline{\mathcal{M}}_{g, n}$. Instead of looking at loci of points $\left[C, x_{1}, \ldots, x_{n}\right] \in \mathcal{M}_{g, n}$ for which the points $x_{1}, \ldots, x_{n}$ become linearly dependent in a suitable embedding of $C$, we can consider the loci where the marked points give rise to an $n$-fold point on a suitable model of $C$. Given $[C] \in \mathcal{M}_{g}$ and $l=(L, V) \in G_{d}^{r}(C)$, we say that the divisor $\Gamma:=x_{1}+\cdots+x_{n}$ is an $n$-fold point for $C$ and $l$ if $\operatorname{dim}\left(V \cap H^{0}\left(L \otimes \mathcal{O}_{C}(-\Gamma)\right)\right) \geq r$.

Definition 4.8. Fix integers $g, r, d, n \geq 1$ such that $\rho(g, r, d)-r(n-1)=-1$. We define the locus of $n$-fold points in $\mathcal{M}_{g, n}$

$\mathfrak{N} \mathfrak{f o r d}{ }_{g, d}^{r}:=\left\{\left[C, x_{1}, \ldots, x_{n}\right] \in \mathcal{M}_{g, n}: \exists L \in W_{d}^{r}(C)\right.$ with $\left.\operatorname{dim} H^{0}\left(L\left(-x_{1}-\cdots-x_{n}\right)\right)=r\right\}$.

We have computed the class of $\mathfrak{N} \mathfrak{f o r d} \mathfrak{d}_{g, d}^{r}$ in the case $r=1$. The calculation is along the same lines as that of the class of $\overline{\mathfrak{L i n}}_{d}^{r}$ in Theorem 4.6

Theorem 4.9. Fix integers $g \geq 1$ and $n \geq 0$ such that $d:=(g+n) / 2 \in \mathbb{Z}$. The class of the compactification of the divisor $\mathfrak{N} \mathfrak{f o r} \mathfrak{d}_{g, d}^{1}$ of $n$-fold points on $\mathcal{M}_{g, n}$ is given by the formula:

$$
\begin{gathered}
\overline{\mathfrak{N} \mathfrak{f o l d}}_{g, d}^{1} \equiv\left(\frac{10 n}{g-2}\left(\begin{array}{c}
g-2 \\
d-1
\end{array}\right)-\frac{n}{g}\left(\begin{array}{l}
g \\
d
\end{array}\right)\right) \lambda+\frac{n-1}{g-1}\left(\begin{array}{c}
g-1 \\
d-1
\end{array}\right) \sum_{j=1}^{n} \psi_{j}-\frac{n}{g-2}\left(\begin{array}{c}
g-2 \\
d-1
\end{array}\right) \delta_{i r r}- \\
-\sum_{t \geq 2} \frac{t\left(n^{2}-g+t g n-t n\right)}{2(g-1)(g-d)}\left(\begin{array}{c}
g-1 \\
d
\end{array}\right) \sum_{|S|=t} \delta_{0: S}-\cdots
\end{gathered}
$$

\section{The Kodaira Dimension of $\overline{\mathcal{M}}_{g, n}$}

In this section we prove Theorem 1.10 . We treat each case individually but for each $g$ we only work out the case of the minimal $n=n(g)$ for which our methods show that $\overline{\mathcal{M}}_{g, n(g)}$ is of general type. From this it follows automatically that $\overline{\mathcal{M}}_{g, n}$ is of general type for all $g \geq g(n)$ (see [Log], Theorem 2.4).

Proof of Theorem 1.10$]\left[\overline{\mathcal{M}}_{4,16}\right]$ and $\left[\overline{\mathcal{M}}_{6,16}\right]$. We consider the divisor $\mathfrak{M r c}_{4,0}^{2}$ on $\overline{\mathcal{M}}_{4,15}$ introduced in Theorem 4.2. We have seen that $\overline{\mathfrak{M r c}}_{4,0}^{2} \equiv-37 \lambda+3 \sum_{j=1}^{1} 5 \psi_{j}+3 \delta_{i r r}-$ 
$7 \sum_{|S|=2} \delta_{0: S}-\cdots$. We consider the maps $\pi_{j}: \overline{\mathcal{M}}_{4,16} \rightarrow \overline{\mathcal{M}}_{4,15}$ obtained by forgetting the marked point labeled by $1 \leq j \leq 16$. Then there exists a constant $\alpha>0$ such that

$$
\sum_{j=1}^{16}\left(\pi_{j}\right)^{*}\left(\overline{\mathfrak{M r c}}_{4,0}^{2}\right) \equiv \alpha\left(-37 \lambda+\frac{45}{16} \sum_{j=1}^{1} 6 \psi_{j}+3 \delta_{i r r}-\frac{13}{2} \sum_{|S|=2} \delta_{0: S}-\cdots\right) .
$$

The class of the Petri divisor on $\overline{\mathcal{M}}_{6}$ being (up to a $>0$ constant) $17 \lambda-2 \delta_{i r r}$, we obtain that $K_{\overline{\mathcal{M}}_{4,16}}$ is big, being a positive combination of $\sum_{j=1}^{16}\left(\pi_{j}\right)^{*}\left(\overline{\mathfrak{M r c}}_{4: 0}^{2}\right)$, the pull-back of the Petri class, an ample class and boundary divisors. The same argument works in the case of $\overline{\mathcal{M}}_{6,16}$ except that we start with the divisor $\overline{\mathfrak{M r c}}_{6,0}^{1}$ on $\overline{\mathcal{M}}_{6,15}$ which then gets pulled back to $\overline{\mathcal{M}}_{6,16}$ in all possible ways.

$\left[\overline{\mathcal{M}}_{5,15}\right]$. On $\overline{\mathcal{M}}_{5,12}$ we have the identity of divisor classes $\overline{\mathfrak{M r c}}_{5,0}^{1} \equiv-13 \lambda+2 \sum_{j=1}^{1} 2 \psi_{j}+$ $\delta_{\text {irr }}-5 \sum_{|S|=2} \delta_{0: S}-\cdots$. Pulling this class back to $\overline{\mathcal{M}}_{5,15}$ in all possible ways by forgetting sets of three marked points, we obtain that the class

$$
-66 \lambda+\frac{42}{5} \sum_{j=1}^{15} \psi_{j}+5 \delta_{i r r}-\cdots
$$

is effective on $\overline{\mathcal{M}}_{5,15}$. Using the Brill-Noether class $8 \lambda-\delta_{\text {irr }}-\cdots$ on $\overline{\mathcal{M}}_{5}$, we get that $K_{\overline{\mathcal{M}}_{5,15}}$ is a big divisor class.

$\left[\overline{\mathcal{M}}_{18,9}\right]$. We use our divisor $\overline{\mathfrak{L i n}}_{24}^{8}$ : there is a positive constant $\alpha$ such that $\alpha \overline{\mathfrak{L i n}}_{24}^{8} \equiv$ $290 \lambda+24 \sum_{j=1}^{9} \psi_{j}-45 \delta_{i r r}-\cdots$. On the other hand the class of the multiple of the Petri divisor $\overline{\mathcal{G P}}_{18,24}^{8}$ on $\overline{\mathcal{M}}_{18}$ is equal to $\frac{302}{45} \lambda-\delta_{0}-\sum_{j=1}^{9} b_{j} \delta_{j}$, where $b_{j}>1$ for $j \geq 1$. It follows that we can write $K_{\overline{\mathcal{M}}_{18,9}}$ as a positive combination of multiples of $\overline{\mathfrak{L i n}}_{24}^{8}$, $\pi^{*}\left(\overline{\mathcal{G P}}_{18,24}^{8}\right)$, boundary divisors and an ample class on $\overline{\mathcal{M}}_{18,9}$.

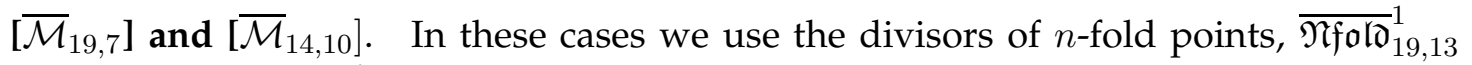
on $\overline{\mathcal{M}}_{19,7}$ and $\overline{\mathfrak{N f o l d}}_{14,12}^{1}$ on $\overline{\mathcal{M}}_{14,10}$ respectively. Using Theorem 4.9 we see that the canonical bundle of $\overline{\mathcal{M}}_{g, n}$ can be written as a positive combination of these divisors, the pull-back of the Brill-Noether divisor from $\overline{\mathcal{M}}_{19}$ and $\overline{\mathcal{M}}_{14}$ respectively, a suitable ample class and boundary divisors.

$\left[\overline{\mathcal{M}}_{15,10}\right]$. We use a slightly different technique. On $\overline{\mathcal{M}}_{15,11}$ we have the divisor $\overline{\mathfrak{N f}} \mathfrak{f} \mathfrak{d}_{15,13}^{1}$ of points $\left[C, x_{1}, \ldots, x_{11}\right]$ such that $x_{1}+\cdots+x_{11}$ appears in a fibre of a $\mathfrak{g}_{13}^{1}$ on $C$. We push this divisor down to $\overline{\mathcal{M}}_{15,10}$ by letting two of the points $x_{j} \in C$ coalesce, that is, we define $E:=\frac{1}{11} \sum_{j=1}^{10}\left(\pi_{j}\right)_{*}\left(\overline{\mathfrak{N f o l d}}_{15,13}^{1} \cdot \delta_{0: j, 11}\right)$, where $\pi_{j}: \overline{\mathcal{M}}_{15,11} \rightarrow \overline{\mathcal{M}}_{15,10}$ forgets the marked point labeled by $j$. It is easy to check using Theorem 4.9 that

$E \equiv 33 \lambda+\frac{396}{5} \sum_{j=1}^{10} \psi_{j}-11 \delta_{i r r}-\cdots\left(\right.$ use that $\left.\left(\pi_{j}\right)_{*}\left(\psi_{j} \cdot \delta_{0: j, 11}\right)=\left(\pi_{j}\right)_{*}\left(\psi_{11} \cdot \delta_{0: j, 11}\right)=0\right)$.

It turns out that $K_{\overline{\mathcal{M}}_{15,11}}$ is in the span of $E, \pi^{*}\left(\overline{\mathcal{M}}_{15,14}^{3}\right)$, an ample class and boundaries. 
$\left[\overline{\mathcal{M}}_{20,6}\right.$ ]. From [Log] Theorem 5.4, one knows that the class $-\lambda+\frac{22}{3} \sum_{j=1}^{6} \psi_{j}-0 \cdot \delta_{i r r}-\cdots$ is effective on $\overline{\mathcal{M}}_{20,6}$. Next, if $\chi_{i, j}: \overline{\mathcal{M}}_{20,6} \rightarrow \overline{\mathcal{M}}_{21}$ denotes the map which associates to a 6-pointed curve of genus 20 a nodal curve of genus 21 obtained by identifying the marked points labeled $i$ and $j$, we also get that the class

$$
\sum_{i<j} \chi_{i, j}^{*}\left(\overline{\mathcal{Z}}_{21,0}\right) \equiv c\left(\frac{2459}{377} \lambda+\frac{1}{3} \sum_{j=1}^{6} \psi_{j}-\delta_{i r r}-\cdots\right),
$$

with $c>0$, is also effective on $\overline{\mathcal{M}}_{20,6}$. The conclusion now follows easily.

\section{REFERENCES}

[AC1] E. Arbarello and M. Cornalba, Calculating cohomology groups of moduli spaces of curves via algebraic geometry, Inst. Hautes Etudes Sci. Publ. Math. 88 (1998), 97-127.

[AC2] E. Arbarello and M. Cornalba, Su una congettura di Petri, Comment. Math. Helv. 56 (1981), 1-38.

[ACGH] E. Arbarello, M. Cornalba, P. Griffiths and J. Harris, Geometry of algebraic curves, Grundlehren der mathematischen Wissenschaften 267, Springer.

[CF] F. Cukierman and L.-Y. Fong, On higher Weierstrass points, Duke Math. J. 62 (1991), 179-203.

[CR] M.C. Chang and Z. Ran, Deformations and smoothings of reducible curves, in: Algebraic Geometry, Bowdoin 1985, Proc. Symph. Pure Math. 46, 63-75.

[EH1] D. Eisenbud and J. Harris, Limit linear series: basic theory, Invent. Math. 85 (1986), 337-371.

[EH2] D. Eisenbud and J. Harris, Irreducibility of some families of linear series with Brill-Noether number -1 , Ann. Scient. Éc. Norm. Sup.(4) 22 (1989), 33-53.

[EH3] D. Eisenbud and J. Harris, The Kodaira dimension of the moduli space of curves of genus $\geq 23$, Invent. Math. 90 (1987), 359-387.

[EH4] D. Eisenbud and J. Harris, A simpler proof of the Gieseker-Petri theorem on special divisors, Invent. Math. 74 (1983), 269-280.

[Est] E. Esteves, Linear systems and ramification points on reducible nodal curves, math.AG/9808069

[Est2] E. Esteves, Compactifying the relative Jacobian over families of reduced curves, Transaction AMS, 353 (2001), 3045-3095.

[F1] G. Farkas, Syzygies of curves and the effective cone of $\overline{\mathcal{M}}_{g}$, math.AG/0503498. Duke Math. J. 135 (2006), 53-98.

[F2] G. Farkas, Gaussian maps, Gieseker-Petri loci and large theta-characteristics, J. reine angew. Math. 581 (2005), 151-173.

[F3] G. Farkas, The geometry of the moduli space of Prym varieties, in preparation.

[FP] G. Farkas and M. Popa, Effective divisors on $\overline{\mathcal{M}}_{g}$, curves on K3 surfaces and the Slope Conjecture, J. Algebraic Geom. 14 (2005), 241-267.

[FMP] G. Farkas, M. Mustata and M. Popa, Divisors on $\mathcal{M}_{g, g+1}$ and the minimal resolution conjecture for points on canonical curves, Ann. Scient. Éc. Norm. Sup. 36 (2003), 558-581.

[FuPr] W. Fulton and P. Pragacz, Schubert varieties and degeneration loci, Springer Lecture Notes in Math. 1689 (1998).

[GG] A. Geramita and A. Gimigliano, Generators for the defining ideal of certain rational surfaces, Duke Math. J. 62 (1991), 61-83.

[GL] M. Green and R. Lazarsfeld, Stability and restrictions of Picard bundles with an application to the normal bundles of elliptic curves, in: Complex Projective Geometry, London Math. Soc. Lecture Notes 179, 149-156.

[Gr] M. Green, Koszul cohomology and the geometry of projective varieties, J. Diff. Geom. 19 (1984), 125-171.

[H1] J. Harris, On the Kodaira dimension of the moduli space of curves II: The even-genus case, Invent. Math. 75 (1984), 437-466.

[HT] J. Harris and L. Tu, Chern numbers of kernel and cokernel bundles, Invent. Math. 75 (1984), 467-475.

[HM] J. Harris and D. Mumford, On the Kodaira dimension of the moduli space of curves, Invent. Math. 67 (1982), 23-88.

[HMo] J. Harris and I. Morrison, Slopes of effective divisors on the moduli space of stable curves, Invent. Math. 99 (1990), 321-355. 
[Kh] D. Khosla, Moduli spaces of curves with linear series and the Slope Conjecture, preprint.

[Laz] R. Lazarsfeld, Brill-Noether-Petri without degenerations, J. Diff. Geom. 23 (1986), 299-307.

[Log] A. Logan, The Kodaira dimension of moduli spaces of curves with marked points, Amer. J. of Math. 125 (2003), 105-138.

[Oss] B. Osserman, A limit linear series moduli scheme, math.AG/0407496

[R] T.G. Room, The geometry of determinantal loci, Cambridge University Press, 1938.

[Ver] A. Verra, The unirationality of the moduli space of curves of genus 14 or lower, Compositio Math. 141 (2005), 1425-1444.

[V1] C. Voisin, Green's generic syzygy conjecture for curves of even genus lying on a K3 surface, J. Eur. Math. Soc. 4 (2002), 363-404.

[V2] C. Voisin, Green's canonical syzygy conjecture for generic curves of odd genus, Compositio Math. 141 (2005), 1163-1190.

Department of Mathematics, University of Texas, Austin, TX 78712

E-mail address: gfarkas@math . utexas.edu 